\title{
GAMMA CLASSES AND QUANTUM COHOMOLOGY OF FANO MANIFOLDS: GAMMA CONJECTURES
}

\author{
SERGEY GALKIN, VASILY GOLYSHEV, AND HIROSHI IRITANI
}

\begin{abstract}
We propose Gamma Conjectures for Fano manifolds which can be thought of as a square root of the index theorem. Studying the exponential asymptotics of solutions to the quantum differential equation, we associate a principal asymptotic class $A_{F}$ to a Fano manifold $F$. We say that $F$ satisfies Gamma Conjecture $I$ if $A_{F}$ equals the Gamma class $\widehat{\Gamma}_{F}$. When the quantum cohomology of $F$ is semisimple, we say that $F$ satisfies Gamma Conjecture $I I$ if the columns of the central connection matrix of the quantum cohomology are formed by $\widehat{\Gamma}_{F} \mathrm{Ch}\left(E_{i}\right)$ for an exceptional collection $\left\{E_{i}\right\}$ in the derived category of coherent sheaves $\mathcal{D}_{\text {coh }}^{b}(F)$. Gamma Conjecture II refines part (3) of Dubrovin's conjecture [18]. We prove Gamma Conjectures for projective spaces and Grassmannians.
\end{abstract}

\section{Contents}

1. Introduction 2

1.1. Gamma class 2

1.2. Gamma conjectures 3

1.3. Gamma Conjectures for Grassmannians 4

1.4. Limit formula and Apery's irrationality 4

1.5. Mirror symmetry 5

1.6. Plan of the paper 6

2. Quantum cohomology, quantum connection and solutions. 6

2.1. Quantum cohomology 6

2.2. Quantum connection 7

2.3. Canonical fundamental solution around $z=\infty \quad 8$

2.4. $U V$-system: semisimple case 9

2.5. Asymptotically exponential flat sections 10

2.6. Mutation and Stokes matrix 13

2.7. Isomonodromic deformation 16

3. Gamma Conjecture I 17

3.1. Property $\mathcal{O} \quad 17$

3.2. Asymptotic solutions along positive real line 18

3.3. Flat sections with the smallest asymptotics 20

3.4. Gamma Conjecture I: statement 20

3.5. The leading asymptotics of dual flat sections 23

3.6. A limit formula for the principal asymptotic class 23

3.7. Apery limit 25

3.8. Quantum cohomology central charges 28

2010 Mathematics Subject Classification. 53D37 (primary), 14N35, 14J45, 14J33, 11 G42 (secondary).

Key words and phrases. Fano varieties; Grassmannians; quantum cohomology; Frobenius manifolds; mirror symmetry; Dubrovin's conjecture; Gamma class; Apery limit; abelian/non-abelian correspondence; quantum Satake principle; derived category of coherent sheaves; exceptional collection; Landau-Ginzburg model. 
4. Gamma Conjecture II 28

4.1. Semiorthonormal basis and Gram matrix 28

4.2. Marked reflection system 29

4.3. Mutation of MRSs 30

4.4. Exceptional collections 34

4.5. Asymptotic basis and MRS of a Fano manifold 35

4.6. Dubrovin's (original) conjecture and Gamma conjecture II 35

4.7. Quantum cohomology central charges 36

5. Gamma conjectures for projective spaces 37

5.1. Quantum connection of projective spaces 37

5.2. Frobenius solutions and Mellin solutions 37

5.3. Monodromy transformation and mutation 39

6. Gamma conjectures for Grassmannians 42

6.1. Statement 42

6.2. Quantum Pieri and quantum Satake 42

6.3. The wedge product of the big quantum connection of $\mathbb{P} \quad 44$

6.4. The wedge product of MRS 48

6.5. MRS of Grassmannian 48

6.6. The wedge product of Gamma basis $\quad 50$

6.7. Gamma Conjecture I for Grassmannians 51

6.8. The abelian/non-abelian correspondence and the Gamma basis 51

Appendix A. $\zeta$-function regularization $\quad 52$

$\begin{array}{ll}\text { References } & 53\end{array}$

\section{INTRODUCTION}

1.1. Gamma class. The Gamma class of a complex manifold $X$ is the cohomology class

$$
\widehat{\Gamma}_{X}=\prod_{i=1}^{n} \Gamma\left(1+\delta_{i}\right) \in H^{\bullet}(X, \mathbb{R})
$$

where $\delta_{1}, \ldots, \delta_{n}$ are the Chern roots of the tangent bundle $T X$ and $\Gamma(x)$ is Euler's Gamma function. A well-known Taylor expansion for the Gamma function implies that it is expanded in the Euler constant $C_{\text {eu }}$ and the Riemann zeta values $\zeta(k), k=2,3, \ldots$ :

$$
\widehat{\Gamma}_{X}=\exp \left(-C_{\mathrm{eu}} c_{1}(X)+\sum_{k \geqslant 2}(-1)^{k}(k-1) ! \zeta(k) \operatorname{ch}_{k}(T X)\right) .
$$

The Gamma class $\widehat{\Gamma}_{X}$ has a loop space interpretation $[52,46]$. Let $\mathcal{L} X$ denote the free loop space of $X$ and consider the locus $X \subset \mathcal{L} X$ of constant loops. The normal bundle $\mathcal{N}$ of $X$ in $\mathcal{L} X$ has a natural $S^{1}$-action (by loop rotation) and splits into the sum $\mathcal{N}_{+} \oplus \mathcal{N}_{-}$of positive and negative representations. By the $\zeta$-function regularization (see [52] and Appendix A), we obtain

$$
\frac{1}{e_{S^{1}}\left(\mathcal{N}_{+}\right)}=\frac{1}{\prod_{k=1}^{\infty} e_{S^{1}}\left(T X \otimes L^{k}\right)} \sim(2 \pi)^{-\frac{\operatorname{dim} X}{2}} z^{-\mu} z^{c_{1}(X)} \widehat{\Gamma}_{X}
$$

where $L$ denotes the $S^{1}$-representation of weight one, $z$ denotes a generator of $H_{S^{1}}^{2}(\mathrm{pt})$ such that $\operatorname{ch}(L)=e^{z}, \mu \in \operatorname{End}\left(H^{\bullet}(X)\right)$ denotes the grading operator defined by $\mu(\phi)=(p-$ 
$\left.\frac{\operatorname{dim} X}{2}\right) \phi$ for $\phi \in H^{2 p}(X)$ and $z^{-\mu}=e^{-(\log z) \mu}$. Therefore $\widehat{\Gamma}_{X}$ can be regarded as a localization contribution from constant maps in Floer theory, cf. Givental's equivariant Floer theory [27].

The Gamma class can be also regarded as a 'square root' of the Todd class (or $\widehat{A}$-class). The Gamma function identity:

$$
\Gamma(1-z) \Gamma(1+z)=\frac{\pi z}{\sin \pi z}=\frac{2 \pi \mathrm{i} z}{1-e^{-2 \pi \mathrm{i} z}} e^{-\pi \mathrm{i} z}
$$

implies that we can factorize the Todd class in the Hirzebruch-Riemann-Roch (HRR) formula as follows:

$$
\begin{aligned}
\chi\left(E_{1}, E_{2}\right) & =\int_{F} \operatorname{ch}\left(E_{1}^{\vee}\right) \cup \operatorname{ch}\left(E_{2}\right) \cup \operatorname{td}{ }_{X} \\
& =\left[\widehat{\Gamma}_{X} \operatorname{Ch}\left(E_{1}\right), \widehat{\Gamma}_{X} \operatorname{Ch}\left(E_{2}\right)\right)
\end{aligned}
$$

where $\chi\left(E_{1}, E_{2}\right)=\sum_{p=0}^{\operatorname{dim} X}(-1)^{p} \operatorname{dim}_{\operatorname{Ext}^{p}}\left(E_{1}, E_{2}\right)$ is the Euler pairing of vector bundles $E_{1}$, $E_{2}, \operatorname{Ch}\left(E_{i}\right)=\sum_{p=0}^{\operatorname{dim} X}(2 \pi \mathrm{i})^{p} \operatorname{ch}_{p}\left(E_{i}\right)$ is the modified Chern character and

$$
[A, B):=\frac{1}{(2 \pi)^{\operatorname{dim} X}} \int_{X}\left(e^{\pi \mathrm{i} c_{1}(X)} e^{\pi \mathrm{i} \mu} A\right) \cup B
$$

is a non-symmetric pairing on $H^{\bullet}(X)$. Geometrically this factorization corresponds to the decomposition $\mathcal{N}=\mathcal{N}_{+} \oplus \mathcal{N}_{-}$of the normal bundle. Recall that Witten and Atiyah [3] derived heuristically the index theorem by identifying the $\widehat{A}$-class with $1 / e_{S^{1}}(\mathcal{N})$. In this sense, the Gamma conjectures can be regarded as a square root of the index theorem.

1.2. Gamma conjectures. The Gamma conjectures relate the quantum cohomology of a Fano manifold and the Gamma class in terms of differential equations. For a Fano manifold $F$, the quantum cohomology algebra $\left(H^{\bullet}(F), \star_{0}\right)$ (at the origin $\tau=0$ ) defines the quantum connection [16]:

$$
\nabla_{z \partial_{z}}=z \frac{\partial}{\partial z}-\frac{1}{z}\left(c_{1}(F) \star_{0}\right)+\mu
$$

acting on $H^{\bullet}(F) \otimes \mathbb{C}\left[z, z^{-1}\right]$. It has a regular singularity at $z=\infty$ and an irregular singularity at $z=0$. Flat sections near $z=\infty$ are constructed by the so-called Frobenius method and can be put into correspondence with cohomology classes in a natural way. Flat sections near $z=0$ are classified by their exponential growth order (along a sector). Our underlying assumption is Property $\mathcal{O}$ (Definition 3.1 .1 ) which roughly says that $c_{1}(F) \star_{0}$ has a simple eigenvalue $T>0$ of the biggest norm. Under Property $\mathcal{O}$, we can single out a flat section $s_{1}(z)$ with the smallest asymptotics $\sim e^{-T / z}$ as $z \rightarrow+0$ along $\mathbb{R}_{>0}$; then we transport the flat section $s_{1}(z)$ to $z=\infty$ and identify the corresponding cohomology class $A_{F}$. We call $A_{F}$ the principal asymptotic class of a Fano manifold. We say that $F$ satisfies Gamma Conjecture $I$ (Conjecture 3.4.3) if $A_{F}$ equals the Gamma class:

$$
A_{F}=\widehat{\Gamma}_{F} .
$$

More generally (under semisimplicity assumption), we can identify a cohomology class $A_{i}$ such that the corresponding flat section has an exponential asymptotics $\sim e^{-u_{i} / z}$ as $z \rightarrow 0$ along a fixed sector (of angle bigger than $\pi$ ) for each eigenvalue $u_{i}$ of $\left(c_{1}(F) \star_{0}\right), i=1, \ldots, N$. These classes $A_{1}, \ldots, A_{N} \in H^{\bullet}(F)$ form a basis which we call the asymptotic basis of $F$. We say that $F$ satisfies Gamma Conjecture II (Conjecture 4.6.1) if the basis can be written as:

$$
A_{i}=\widehat{\Gamma}_{F} \operatorname{Ch}\left(E_{i}\right)
$$


for a certain exceptional collection $\left\{E_{1}, \ldots, E_{N}\right\}$ of the derived category $\mathcal{D}_{\text {coh }}^{b}(F)$. Gamma Conjecture I says that the exceptional object $\mathcal{O}_{F}$ corresponds to the biggest real positive eigenvalue $T$ of $\left(c_{1}(F) \star_{0}\right)$.

The quantum connection has an isomonodromic deformation over the cohomology group $H^{\bullet}(F)$. By Dubrovin's theory [16, 17], the asymptotic basis $\left\{A_{i}\right\}$ changes by mutation

$$
\left(A_{1}, \ldots, A_{i}, A_{i+1}, \ldots, A_{N}\right) \longrightarrow\left(A_{1}, \ldots, A_{i+1}, A_{i}-\left[A_{i}, A_{i+1}\right) A_{i+1}, \ldots, A_{N}\right)
$$

when the eigenvalues $u_{i}$ and $u_{i+1}$ are interchanged (see Figure 8). Via the HRR formula (1.1.2) this corresponds to a mutation of the exceptional collection $\left\{E_{i}\right\}$. The braid group acts on the set of asymptotic bases by mutation and we formulate this in terms of a marked reflection system (MRS) in §4.2. Note that Gamma Conjecture II implies (part (2) of) Dubrovin's conjecture [18] (see $\S 4.6$ ), which says that the Stokes matrix $S_{i j}=\left[A_{i}, A_{j}\right.$ ) of the quantum connection equals the Euler pairing $\chi\left(E_{i}, E_{j}\right)$. While we were writing this paper, we were informed that Dubrovin [19] gave a new formulation of his conjecture that includes Gamma Conjecture II above.

1.3. Gamma Conjectures for Grassmannians. In this paper, we establish Gamma conjectures for projective spaces and for Grassmannians. The Gamma Conjectures for $\mathbb{P}^{n}$ were implicit but essentially shown in the work of Dubrovin [17]; they also follow from mirror symmetry computations in $[44,45,49]$. In $\S 5$, we give an elementary proof of the following theorem.

Theorem 1.3.1 (Theorem 5.0.1). Gamma Conjectures I and II hold for the projective space $\mathbb{P}=\mathbb{P}^{N-1}$. An asymptotic basis of $\mathbb{P}$ is formed by mutations of the Gamma basis $\widehat{\Gamma}_{\mathbb{P}} \mathrm{Ch}(\mathcal{O}(i))$ associated to Beilinson's exceptional collection $\{\mathcal{O}(i): 0 \leqslant i \leqslant N-1\}$.

We deduce the Gamma Conjectures for Grassmannians $G(r, N)$ from the truth of the Gamma Conjectures for projective spaces. The main ingredient in the proof is quantum Satake principle [32] or abelian/non-abelian correspondence [9, 14], which says that the quantum connection of $G(r, N)$ is the $r$-th wedge of the quantum connection of $\mathbb{P}^{N-1}$.

Theorem 1.3.2 (Theorem 6.1.1). Gamma Conjectures I and II hold for Grassmannians $\mathbb{G}=$ $G(r, N)$. An asymptotic basis of $\mathbb{G}$ is formed by mutations of the Gamma basis $\widehat{\Gamma}_{\mathbb{G}} \mathrm{Ch}\left(S^{\nu} V^{*}\right)$ associated to Kapranov's exceptional collection $\left\{S^{\nu} V^{*}: \nu \subset r \times(N-r)\right.$-box $\}$, where $V$ is the tautological bundle and $S^{\nu}$ is the Schur functor.

The grounds are not sufficient at the moment to claim the Gamma Conjectures for all Fano varieties. However the following cases are established. Golyshev and Zagier have announced a proof of Gamma Conjecture I for Fano 3-folds of Picard rank one. In a separate paper [25], we use mirror symmetry to show Gamma Conjectures for certain toric manifolds or toric complete intersections; we will also discuss the compatibility of Gamma Conjecture I with taking hyperplane sections (quantum Lefschetz). Together with the method in the present paper (i.e. compatibility with abelian/non-abelian correspondence), the current techniques would allow us to prove Gamma Conjectures for a wide class of Fano manifolds.

1.4. Limit formula and Apery's irrationality. Let $J(t)=J\left(-\log (t) K_{F}\right)$ denote Givental's $J$-function (3.6.7) restricted to the anti-canonical line $\mathbb{C} c_{1}(F)$. This is a cohomologyvalued solution to the quantum differential equation. We show the following (continuous and discrete) limit formulae: 
Theorem 1.4.1 (Corollary 3.6.9). Suppose that a Fano manifold $F$ satisfies Property $\mathcal{O}$ and Gamma Conjecture I. Then the Gamma class of $F$ can be obtained as the limit of the ratio of the $J$-function

$$
\widehat{\Gamma}_{F}=\lim _{t \rightarrow+\infty} \frac{J(t)}{\langle[\mathrm{pt}], J(t)\rangle} .
$$

Theorem 1.4.2 (see Theorem 3.7.1 for precise statements). Under the same assumptions as above, the primitive part of the Gamma class can be obtained as the following discrete limit (assuming the limit exists):

$$
\left\langle\gamma, \widehat{\Gamma}_{F}\right\rangle=\lim _{n \rightarrow \infty} \frac{\left\langle\gamma, J_{r n}\right\rangle}{\left\langle[\mathrm{pt}], J_{r n}\right\rangle}
$$

for every $\gamma \in H .(F)$ with $c_{1}(F) \cap \gamma=0$. Here we write $J(t)=e^{c_{1}(F) \log t} \sum_{n=0}^{\infty} J_{r n} t^{r n}$ with $r$ the Fano index.

Discrete limits similar to (1.4.3) were studied by Almkvist-van Straten-Zudilin [2] in the context of Calabi-Yau differential equations and are called Apery limits (or Apery constants). Golyshev [31] and Galkin [23] studied the limits (1.4.3) for Fano manifolds. In fact, these limits are related to famous Apery's proof of the irrationality of $\zeta(2)$ and $\zeta(3)$. An Apery limit of the Grassmannian $G(2,5)$ gives a fast approximation of $\zeta(2)$ and an Apery limit of the orthogonal Grassmannian $\operatorname{OGr}(5,10)$ gives a fast approximation of $\zeta(3)$; they prove the irrationality of $\zeta(2)$ and $\zeta(3)$. It would be extremely interesting to find a Fano manifold whose Apery limits give fast approximations of other zeta values.

1.5. Mirror symmetry. Gamma Conjectures are closely related to mirror symmetry and relevant phenomena have been observed since its early days. The following references serve as motivation to the Gamma conjectures.

- The Gamma class of a Calabi-Yau threefold $X$ is given by $\widehat{\Gamma}_{X}=1-\frac{\pi^{2}}{6} c_{2}(X)-$ $\zeta(3) c_{3}(X)$; the number $\zeta(3) \chi(X)$ appeared in the computation of mirror periods by Candelas et al. [13]; it also appeared in the conifold period formula of van Enckevortvan Straten [20].

- Libgober [51] found the (inverse) Gamma class from hypergeometric solutions to the Picard-Fuchs equation of the mirror, inspired by the work of Hosono et al. [42].

- Kontsevich's homological mirror symmetry suggests that the monodromy of the Picard-Fuchs equation of mirrors should be related to Auteq $\left(\mathcal{D}_{\text {coh }}^{b}(X)\right)$. In the related works of Horja [40], Borisov-Horja [11] and Hosono [41], Gamma/hypergeometric series play an important role.

- In the context of Fano/Landau-Ginzburg mirror symmetry, Iritani [44, 45] and Katzarkov-Kontsevich-Pantev [49] introduced a rational or integral structure of quantum connection in terms of the Gamma class, by shifting the natural integral structure of the Landau-Ginzburg model given by Lefschetz thimbles.

Although not directly related to mirror symmetry, we remark that the Gamma class also appears in a recent progress $[39,36]$ in physics on the sphere/hemisphere partition functions.

Remark 1.5.1. In their studies of a 'generalized' Hodge structure (TERP/nc-Hodge structure), Hertling-Sevenheck [38] and Katzarkov-Kontsevich-Pantev [49] discussed the compatibility of the Stokes structure with the real/rational structure. Our Gamma conjecture can be regarded as an explicit Fano-version of the following more general conjecture: the $\widehat{\Gamma}$-integral structure in quantum cohomology should be compatible with the Stokes structure. 
1.6. Plan of the paper. In $\S 2$, we review quantum cohomology and quantum differential equation for Fano manifolds. We explain asymptotically exponential flat sections, their mutation and Stokes matrices. The content in this section is mostly taken from Dubrovin's work $[16,17]$, but we make the following technical refinement: we carefully deal with the case where the quantum cohomology is semisimple but the Euler multiplication $\left(E \star_{\tau}\right)$ has repeated eigenvalues (see Propositions 2.5.1 and 2.6.4). In particular, we show that a semisimple point $\tau$ is not a turning point even when $E \star_{\tau}$ has multiple eigenvalues. We need this case since it happens for Grassmannians. In $\S 3$, we formulate Property $\mathcal{O}$ and Gamma Conjecture I. We also prove limit formulae for the principal asymptotic class. In $\S 4$, we formulate Gamma Conjecture II and explain a relationship to (original) Dubrovin's conjecture. In $\S 5$, we prove Gamma Conjectures for projective spaces. In $\S 6$, we deduce the Gamma Conjectures for Grassmannians from the truth of the Gamma Conjectures for projective spaces. Main tools in the proof are isomonodromic deformation and quantum Satake principle. For this purpose we extend quantum Satake principle [32] to big quantum cohomology (Theorem 6.3.1) using abelian/non-abelian correspondence [9, 14, 50].

\section{Quantum COHOMOlOgY, QUANTUm CONnECTION AND SOlUtions.}

In this section we discuss background material on quantum cohomology and quantum connection of a Fano manifold. Quantum connection is defined as a meromorphic flat connection of the trivial cohomology bundle over the $z$-plane, with singularities at $z=0$ and $z=\infty$. We discuss two fundamental solutions associated to the regular singularity $(z=\infty)$ and the irregular singularity $(z=0)$. Under the semisimplicity assumption, we discuss mutations and Stokes matrices.

2.1. Quantum cohomology. Let $F$ be a Fano manifold, i.e. a smooth projective variety such that the anticanonical line bundle $\omega_{F}^{-1}=\operatorname{det}(T F)$ is ample. Let $H^{\bullet}(F)=H^{\text {even }}(F ; \mathbb{C})$ denote the even part of the Betti cohomology group over $\mathbb{C}$. For $\alpha_{1}, \alpha_{2}, \ldots, \alpha_{n} \in H^{\bullet}(F)$, let $\left\langle\alpha_{1}, \alpha_{2}, \ldots, \alpha_{n}\right\rangle_{0, n, d}^{F}$ denote the genus-zero $n$ points Gromov-Witten invariant of degree $d \in H_{2}(F ; \mathbb{Z})$, see e.g. [54]. Informally speaking, this counts the (virtual) number of rational curves in $F$ which intersect the Poincaré dual cycles of $\alpha_{1}, \ldots, \alpha_{n}$. It is a rational number when $\alpha_{1}, \ldots, \alpha_{n} \in H^{\bullet}(F ; \mathbb{Q})$. The quantum product $\alpha_{1} \star_{\tau} \alpha_{2} \in H^{\bullet}(F)$ of two classes $\alpha_{1}, \alpha_{2} \in H^{\bullet}(F)$ with parameter $\tau \in H^{\bullet}(F)$ is given by

$$
\left(\alpha_{1} \star_{\tau} \alpha_{2}, \alpha_{3}\right)_{F}=\sum_{d \in \operatorname{Eff}(F)} \sum_{n=0}^{\infty} \frac{1}{n !}\left\langle\alpha_{1}, \alpha_{2}, \alpha_{3}, \tau, \ldots, \tau\right\rangle_{0,3+n, d}^{F}
$$

where $(\alpha, \beta)_{F}=\int_{F} \alpha \cup \beta$ is the Poincaré pairing and $\operatorname{Eff}(F) \subset H_{2}(F ; \mathbb{Z})$ is the set of effective curve classes. The quantum product is associative and commutative, and recovers the cup product in the limit where $\operatorname{Re}\left(\int_{d} \tau\right) \rightarrow-\infty$ for all non-zero effective curve classes $d$. It is not known if the quantum product $\star_{\tau}$ converges in general, however it does for all the examples in this paper (see also Remark 2.1.2). The quantum product $\star_{\tau}$ with $\tau \in H^{2}(F)$ is called the small quantum product; for general $\tau \in H^{\bullet}(F)$ it is called the big quantum product.

We are particularly interested in the quantum product $\star_{0}$ specialized to $\tau=0$. An effective class $d$ contributing to the sum

$$
\sum_{d \in \operatorname{Eff}(F)}\left\langle\alpha_{1}, \alpha_{2}, \alpha_{3}\right\rangle_{0,3, d}^{F}
$$


has to satisfy $\frac{1}{2} \sum_{i=1}^{3} \operatorname{deg} \alpha_{i}=\operatorname{dim} F+c_{1}(F) \cdot d$ and there are only finitely many such $d$ when $F$ is Fano. Therefore the specialization at $\tau=0$ makes sense.

Remark 2.1.2. Writing $\tau=h+\tau^{\prime}$ with $h \in H^{2}(F)$ and $\tau^{\prime} \in \bigoplus_{p \neq 1} H^{2 p}(F)$ and using the divisor axiom in Gromov-Witten theory, we have:

$$
\left(\alpha_{1} \star_{\tau} \alpha_{2}, \alpha_{3}\right)_{F}=\sum_{d \in \operatorname{Eff}(F)} \sum_{n=0}^{\infty} \frac{1}{n !}\left\langle\alpha_{1}, \alpha_{2}, \alpha_{3}, \tau^{\prime}, \ldots, \tau^{\prime}\right\rangle_{0, n+3, d}^{F} e^{\int_{d} h} .
$$

Therefore the quantum product (2.1.1) makes sense as a formal power series in $\tau^{\prime}$ and the exponentiated $H^{2}$-variables $e^{h_{1}}, \ldots, e^{h_{r}}$, where we write $h=h_{1} p_{1}+\cdots+h_{r} p_{r}$ by choosing a nef basis $\left\{p_{1}, \ldots, p_{r}\right\}$ of $H^{2}(X ; \mathbb{Z})$.

2.2. Quantum connection. Following Dubrovin [16, 18, 17], we introduce a meromorphic flat connection associated to the quantum product. Consider a trivial vector bundle $H^{\cdot}(F) \times \mathbb{P}^{1} \rightarrow \mathbb{P}^{1}$ over $\mathbb{P}^{1}$ and fix an inhomogeneous co-ordinate $z$ on $\mathbb{P}^{1}$. Define the quantum connection $\nabla$ on the trivial bundle by the formula

$$
\nabla_{z \partial_{z}}=z \frac{\partial}{\partial z}-\frac{1}{z}\left(c_{1}(F) \star_{0}\right)+\mu
$$

where $\mu \in \operatorname{End}\left(H^{\bullet}(F)\right)$ is the grading operator defined by $\left.\mu\right|_{H^{2 p}(F)}=\left(p-\frac{\operatorname{dim} F}{2}\right) \operatorname{id}_{H^{2 p}(F)}$ $(\operatorname{dim} F$ denotes the complex dimension of $F)$. The connection is smooth away from $\{0, \infty\}$; the singularity at $z=\infty$ is regular (or more precisely logarithmic) and the singularity at $z=0$ is irregular. The quantum connection preserves the Poincaré pairing in the following sense: we have

$$
z \frac{\partial}{\partial z}\left(s_{1}(-z), s_{2}(z)\right)=\left(\left(\nabla_{z \partial_{z}} s_{1}\right)(-z), s_{2}(z)\right)+\left(s_{1}(-z), \nabla_{z \partial_{z}} s_{2}(z)\right)
$$

for $s_{1}, s_{2} \in H^{\bullet}(F) \otimes \mathbb{C}\left[z, z^{-1}\right]$. Here we need to flip the sign of $z$ for the first entry $s_{1}$.

What is important in Dubrovin's theory is the fact that $\nabla$ admits an isomonodromic deformation over $H^{\bullet}(F)$. Suppose that $\star_{\tau}$ converges on a region $B \subset H^{\bullet}(F)$. Then the above connection is extended to a meromorphic flat connection on $H^{\bullet}(F) \times\left(B \times \mathbb{P}^{1}\right) \rightarrow\left(B \times \mathbb{P}^{1}\right)$ as follows:

$$
\begin{aligned}
\nabla_{\alpha} & =\partial_{\alpha}+\frac{1}{z}\left(\alpha \star_{\tau}\right) \quad \alpha \in H^{\bullet}(F) \\
\nabla_{z \partial_{z}} & =z \frac{\partial}{\partial z}-\frac{1}{z}\left(E \star_{\tau}\right)+\mu
\end{aligned}
$$

with $(\tau, z)$ a point on the base $B \times \mathbb{P}^{1}$. Here $\partial_{\alpha}$ denotes the directional derivative in the direction of $\alpha$ and

$$
E=c_{1}(F)+\sum_{i=1}^{N}\left(1-\frac{1}{2} \operatorname{deg} \phi_{i}\right) \tau^{i} \phi_{i}
$$

is the Euler vector field, where we write $\tau=\sum_{i=1}^{N} \tau^{i} \phi_{i}$ by choosing a homogeneous basis $\left\{\phi_{1}, \ldots, \phi_{N}\right\}$ of $H^{\bullet}(F)$. We refer to this extension as the big quantum connection. The Poincaré pairing $(\cdot, \cdot)_{F}$ is flat with respect to the big quantum connection $\nabla$, i.e. $\partial_{\alpha}\left(s_{1}(\tau,-z), s_{2}(\tau, z)\right)=\left(\left(\nabla_{\alpha} s_{1}\right)(\tau,-z), s_{2}(\tau, z)\right)+\left(s_{1}(\tau,-z), \nabla_{\alpha} s_{2}(\tau, z)\right)$.

Remark 2.2.4. The connection in the $z$-direction can be identified with the connection in the anticanonical direction after an appropriate rescaling. Consider the quantum product $\star_{\tau}$ 
restricted to the anticanonical line $\tau=c_{1}(F) \log t, t \in \mathbb{C}^{\times}$:

$$
\left(\alpha_{1} \star_{c_{1}(F) \log t} \alpha_{2}, \alpha_{3}\right)_{F}=\sum_{d \in \operatorname{Eff}(F)}\left\langle\alpha_{1}, \alpha_{2}, \alpha_{3}\right\rangle_{0,3, d} t^{c_{1}(F) \cdot d} .
$$

This is a polynomial in $t$ since $F$ is Fano, and coincides with $\star_{0}$ when $t=1$. It can be recovered from the product $\star_{0}$ by the formula:

$$
\left(\alpha \star_{c_{1}(F) \log t}\right)=t^{\operatorname{deg} \alpha / 2} t^{-\mu}\left(\alpha \star_{0}\right) t^{\mu} .
$$

The quantum connection restricted to the anticanonical line and $z=1$ is

$$
\left.\nabla_{c_{1}(F)}\right|_{z=1}=t \frac{\partial}{\partial t}+\left(c_{1}(F) \star_{c_{1}(F) \log t}\right)
$$

On the other hand we have

$$
z^{\mu}\left[\nabla_{z \partial_{z}}\right]_{\tau=0} z^{-\mu}=z \frac{\partial}{\partial z}-\left(c_{1}(F) \star_{-c_{1}}(F) \log z\right)
$$

Therefore, the connections $\left.\nabla_{c_{1}(F)}\right|_{z=1}$ and $\left.\nabla_{z \partial_{z}}\right|_{\tau=0}$ are gauge equivalent via $z^{\mu}$ under the change of variables $t=z^{-1}$.

2.3. Canonical fundamental solution around $z=\infty$. We consider the connection $\nabla$ (2.2.1) defined by the quantum product $\star_{0}$ at $\tau=0$. We have a (well-known) canonical fundamental solution $S(z) z^{-\mu} z^{\rho}$ for $\nabla$ associated to the regular singular point $z=\infty$.

Proposition 2.3.1. There exists a unique holomorphic function $S: \mathbb{P}^{1} \backslash\{0\} \rightarrow \operatorname{End}\left(H^{\bullet}(F)\right)$ with $S(\infty)=\operatorname{id}_{H^{\bullet}(F)}$ such that

$$
\begin{aligned}
& \nabla\left(S(z) z^{-\mu} z^{\rho} \alpha\right)=0 \quad \text { for all } \alpha \in H^{\bullet}(F) ; \\
& T(z)=z^{\mu} S(z) z^{-\mu} \text { is regular at } z=\infty \text { and } T(\infty)=\operatorname{id}_{H^{\bullet}(F)},
\end{aligned}
$$

where $\rho=\left(c_{1}(F) \cup\right) \in \operatorname{End}\left(H^{\bullet}(F)\right)$ and we define $z^{-\mu}=\exp (-\mu \log z), z^{\rho}=\exp (\rho \log z)$. Moreover we have

$$
(S(-z) \alpha, S(z) \beta)_{F}=(\alpha, \beta)_{F} \quad \alpha, \beta \in H^{\bullet}(F) .
$$

Proof. The endomorphism $S(z)$ is a gauge transformation giving the Levelt normal form (see e.g. [57, Exercise 2.20]) of the connection $\nabla$ near $z=\infty$. A similar fundamental solution was given by Dubrovin for a general Frobenius manifold [17, Lemma 2.5, 2.6]; we also have an explicit formula in terms of Gromov-Witten invariants (see Remark 2.3.2 below). Note that $S(z)$ in our case satisfies the additional properties that $T(z)=z^{\mu} S(z) z^{-\mu}$ is regular at $z=\infty$ and that $T(\infty)=\mathrm{id}$, which ensure the uniqueness of $S$. We give a construction of $S(z)$ to verify these points.

Consider the equivalent differential equation $\nabla\left(z^{-\mu} T(z) z^{\rho} \alpha\right)=0$ for $T(z)=z^{\mu} S(z) z^{-\mu}$ with the initial condition $T(\infty)=$ id. The differential equation for $T$ reads:

$$
z \frac{\partial}{\partial z} T(z)-\frac{1}{z} z^{\mu}\left(c_{1}(F) \star_{0}\right) z^{-\mu} T(z)+T(z) \rho=0 .
$$

Expand:

$$
\begin{aligned}
T(z) & =\mathrm{id}+T_{1} z^{-1}+T_{2} z^{-2}+T_{3} z^{-3}+\cdots \\
\left(c_{1}(F) \star_{0}\right) & =G_{0}+G_{1}+G_{2}+\cdots \quad(\text { finite sum })
\end{aligned}
$$


where $G_{k} \in \operatorname{End}\left(H^{\bullet}(F)\right)$ is an endomorphism of degree $1-k$, i.e. $z^{\mu} G_{k} z^{-\mu}=z^{1-k} G_{k}$ and $G_{0}=c_{1}(F) \cup=\rho$. The above equation is equivalent to the system of equations:

$$
\begin{aligned}
0 & =\rho-\rho \\
0 & =T_{1}+G_{1}+\left[\rho, T_{1}\right] \\
& \vdots \\
0 & =m T_{m}+G_{m}+G_{m-1} T_{1}+\cdots+G_{1} T_{m-1}+\left[\rho, T_{m}\right] .
\end{aligned}
$$

These equations can be solved recursively for $T_{1}, T_{2}, T_{3}, \ldots$ because the map $X \mapsto m X+[\rho, X]$ is invertible (since $\rho$ is nilpotent). One can easily show the convergence of $T(z)$.

By construction, $T_{k}$ is an endomorphism of degree $\geqslant(1-k)$ and hence $z^{-k} z^{-\mu} T_{k} z^{\mu}$ contains only negative powers in $z$ for $k \geqslant 1$. Therefore $S(z)=z^{-\mu} T(z) z^{\mu}$ is regular at $z=\infty$ and satisfies $S(\infty)=\mathrm{id}$.

Finally we show $(S(-z) \alpha, S(z) \beta)_{F}=(\alpha, \beta)_{F}$. We claim that

$$
\left(S(-z)\left(e^{\pi \mathrm{i}} z\right)^{-\mu}\left(e^{\pi \mathrm{i}} z\right)^{\rho} \alpha, S(z) z^{-\mu} z^{\rho} \beta\right)_{F}=\left(e^{-\pi \mathrm{i} \mu} e^{\pi \mathrm{i} \rho} \alpha, \beta\right)_{F} .
$$

Because $\nabla$ preserves the Poincaré pairing (2.2.2), the left-hand side is independent of $z$. On the other hand, the left-hand side equals

$$
\left(e^{-\pi \mathfrak{i} \mu} T(-z)\left(e^{\pi \dot{\mathrm{i}}} z\right)^{\rho} \alpha, T(z) z^{\rho} \beta\right)_{F}
$$

and can be expanded in $\mathbb{C} \llbracket z^{-1} \rrbracket[\log z]$. The constant term of the Taylor expansion in $z^{-1}$ and $\log z$ equals the right-hand side. The claim follows. Replacing $\alpha, \beta$ with $\left(e^{\pi \mathrm{i}} z\right)^{-\rho}\left(e^{\pi \mathrm{i}} z\right)^{\mu} \alpha$ and $z^{-\rho} z^{\mu} \beta$, we arrive at the conclusion.

Remark 2.3.2 ([16, 28, 56, 45]). The fundamental solution $S(z)$ is given by descendant Gromov-Witten invariants. Let $\psi$ denote the first Chern class of the universal cotangent line bundle over $\bar{M}_{0,2}(F, d)$ at the first marking. Then we have:

$$
(S(z) \alpha, \beta)_{F}=(\alpha, \beta)_{F}+\sum_{m \geqslant 0} \frac{1}{z^{m+1}} \sum_{d \in \operatorname{Eff}(F) \backslash\{0\}}(-1)^{m+1}\left\langle\alpha \psi^{m}, \beta\right\rangle_{0,2, d}^{F} .
$$

Here again the summation in $d$ is finite (for a fixed $m$ ) because $F$ is Fano. A similar fundamental solution exists for the isomonodromic deformation of $\nabla$ associated to the big quantum cohomology. The big quantum connection $\nabla$ over $H^{\bullet}(F) \times \mathbb{P}^{1}$ admits a fundamental solution of the form $S(\tau, z) z^{-\mu} z^{\rho}$ extending the one in Proposition 2.3.1 such that

$$
\nabla\left(S(\tau, z) z^{-\mu} z^{\rho} \alpha\right)=0, \quad S(\tau, \infty)=\mathrm{id}, \quad(S(\tau,-z) \alpha, S(\tau, z) \beta)_{F}=(\alpha, \beta)_{F} .
$$

Here $z^{\mu} S(\tau, z) z^{-\mu}$ is not necessarily regular at $z=\infty$ for $\tau \notin H^{2}(F)$ (cf. Lemma 6.5.3).

2.4. $U V$-system: semisimple case. Suppose that the quantum product $\star_{\tau}$ is convergent and semisimple at some $\tau \in H^{\bullet}(F)$. The semisimplicity means that the algebra $\left(H^{\bullet}(F), \star_{\tau}\right)$ is isomorphic to a direct sum of $\mathbb{C}$ as a ring. Let $\psi_{1}, \ldots, \psi_{N}$ denote the idempotent basis of $H^{*}(F)$ such that

$$
\psi_{i} \star_{\tau} \psi_{j}=\delta_{i, j} \psi_{i}
$$

where $N=\operatorname{dim} H^{\bullet}(F)$. Let $\Psi_{i}:=\psi_{i} / \sqrt{\left(\psi_{i}, \psi_{i}\right)_{F}}, i=1, \ldots, N$ be the normalized idempotents. They form an orthonormal basis and are unique up to sign. We write

$$
\Psi=\left(\Psi_{1}, \ldots, \Psi_{N}\right)
$$


for the matrix with the column vectors $\Psi_{1}, \ldots, \Psi_{N}$. We regard $\Psi$ as a homomorphism $\mathbb{C}^{N} \rightarrow$ $H^{\bullet}(F)$. Let $u_{1}, \ldots, u_{N}$ be the eigenvalues of $\left(E \star_{\tau}\right)$ given by $E \star_{\tau} \Psi_{i}=u_{i} \Psi_{i}$. Define $U$ to be the diagonal matrix with entries $u_{1}, \ldots, u_{N}$ :

$$
U=\left(\begin{array}{llll}
u_{1} & & & \\
& u_{2} & & \\
& & \ddots & \\
& & & u_{N}
\end{array}\right)
$$

We allow $E \star_{\tau}$ (or $U$ ) to have repeated eigenvalues. By the constant gauge transformation $\Psi$, the quantum connection $\nabla_{z \partial_{z}}(2.2 .3)$ is transformed to the connection

$$
\Psi^{*} \nabla_{z \partial_{z}}=z \frac{\partial}{\partial z}-\frac{1}{z} U+V
$$

with $V=\Psi^{-1} \mu \Psi$. We call this the $U V$-system, cf. [16, Lecture 3]. Notice that the semisimplicity is an open condition: when $\tau$ varies $^{1}$, the matrices $\Psi$ and $U$ depends analytically on $\tau$ as far as $\star_{\tau}$ is semisimple. Moreover $\tau \mapsto\left(u_{1}, \ldots, u_{N}\right)$ gives a local co-ordinate system on $H^{\bullet}(F)$ and one has $\psi_{i}=\frac{\partial \tau}{\partial u_{i}}$ (see [17, Lecture 3]). The $U V$-system is extended in the $H^{\bullet}(F)$-direction as follows [17, Lemma 3.2]:

$$
\Psi^{*} \nabla_{\partial_{u_{i}}}=\frac{\partial}{\partial u_{i}}+\frac{1}{z} E_{i}+V_{i}
$$

where $E_{i}=\operatorname{diag}[0, \ldots, 0, \stackrel{i \text { th }}{1}, 0, \ldots, 0]$ and $V_{i}=\Psi^{-1} \partial_{u_{i}} \Psi$.

Lemma 2.4.4. The matrix $V=\left(V_{i j}\right)$ is anti-symmetric $V_{i j}=-V_{j i}$. Moreover, $V_{i j}=0$ whenever $u_{i}=u_{j}$.

Proof. The anti-symmetricity of $V$ follows from the fact that $\mu$ is skew-adjoint: $(\mu \alpha, \beta)_{F}=$ $-(\alpha, \mu \beta)_{F}$ and that $\Psi_{1}, \ldots, \Psi_{N}$ are orthonormal. To see the latter statement, we use the isomonodromic deformation (2.4.2)-(2.4.3). The flatness of $\Psi^{*} \nabla$ implies:

$$
\left[E_{i}, V\right]=\left[V_{i}, U\right]
$$

and it follows that $V_{i j}=\left(u_{j}-u_{i}\right)\left(V_{i}\right)_{i j}$ if $i \neq j$. Therefore $V_{i j}=0$ if $i \neq j$ and $u_{i}=u_{j}$. If $i=j, V_{i j}=0$ by the anti-symmetricity.

2.5. Asymptotically exponential flat sections. Under semisimplicity assumption, we will construct a basis of flat sections for the quantum connection near the irregular singular point $z=0$ which have exponential asymptotics $\sim e^{-u_{i} / z}$ as $z \rightarrow 0$ along an angular sector.

The so-called Hukuhara-Turrittin theorem [62, 43, 53, 63], [65, Theorem 19.1], [57, II, 5.d], $[38, \S 8]$ says that $\nabla_{z \partial_{z}}$ admits, after a change ${ }^{2}$ of variables $z=w^{r}$ with $r \in \mathbb{Z}_{>0}$, a fundamental matrix solution around $z=0$ of the form:

$$
P(w) w^{C} e^{\Lambda\left(w^{-1}\right)}
$$

where $\Lambda\left(w^{-1}\right)$ is a diagonal matrix whose entries are polynomials in $w^{-1}, C$ is a constant matrix, and $P(w)$ is an invertible matrix-valued function having an asymptotic expansion $P(w) \sim P_{0}+P_{1} w+P_{2} w^{2}+\cdots$ as $|w| \rightarrow 0$ in an angular sector.

\footnotetext{
${ }^{1}$ If quantum cohomology is not known to converge except at $\tau$, we can work with the formal neighbourhood of $\tau$ in $H^{\bullet}(F)$ in the following discussion.

${ }^{2}$ In view of mirror symmetry, we might hope that the order $r$ of ramification equals 1 for a wide class of Fano manifolds; this is referred to as $\nabla$ being of "exponential type" in [49, Definition 2.12].
} 
When the eigenvalues $u_{1}, \ldots, u_{N}$ of $E_{\star_{\tau}}$ are pairwise distinct, the fundamental solution takes a simpler form: we have $r=1, z=w$ and $\Lambda\left(w^{-1}\right)=-U z^{-1}$ with $U=\operatorname{diag}\left[u_{1}, \ldots, u_{N}\right]$. This case has been studied by many people, including Wasow [65, Theorem 12.3], BalserJurkat-Lutz [4, Theorem A, Proposition 7], [5] (for general irregular connections) and Dubrovin [17, Lectures 4, 5] (in the context of Frobenius manifolds). Bridgeland-ToledanoLaredo [12] studied the case where $E_{\star_{\tau}}$ is semisimple but has repeated eigenvalues. Under a certain condition $[12, \S 8(\mathrm{~F})]$, they showed that one can take $r=1$ and $\Lambda\left(w^{-1}\right)=-U / z$; their condition $(\mathrm{F})$ is ensured, in our setting, by Lemma 2.4.4. We extend the results in $[4,5,17,12]$ to isomonodromic deformation (where $u_{1}, \ldots, u_{N}$ are not necessarily distinct) and show that both formal and actual solutions depend analytically on $\tau$.

We say that a phase $\phi \in \mathbb{R}$ (or a direction $e^{\mathbf{i} \phi} \in S^{1}$ ) is admissible ${ }^{3}$ for a multiset $\left\{u_{1}, \ldots, u_{N}\right\} \subset \mathbb{C}$ if $e^{-i \phi}\left(u_{i}-u_{j}\right) \notin \mathbb{R}_{>0}$ holds for every $(i, j)$, i.e. $e^{i \phi}$ is not parallel to any non-zero difference $u_{i}-u_{j}$.

Proposition 2.5.1. Assume that the quantum product $\star_{\tau}$ is analytic and semisimple in a neighbourhood $B$ of $\tau_{0} \in H^{\bullet}(F)$. Consider the big quantum connection $\nabla(2.2 .3)$ over $B \times \mathbb{P}^{1}$. Let $\phi \in \mathbb{R}$ be an admissible phase for the spectrum $\left\{u_{1,0}, \ldots, u_{N, 0}\right\}$ of $\left(E_{\star_{\tau_{0}}}\right)$. Then, shrinking $B$ if necessary, we have an analytic fundamental solution $Y_{\tau}(z)=\left(y_{1}(\tau, z), \ldots, y_{N}(\tau, z)\right)$ for $\nabla$ and $\epsilon>0$ such that

$$
Y_{\tau}(z) e^{U / z} \rightarrow \Psi \quad \text { as } z \rightarrow 0 \text { in the sector }|\arg z-\phi|<\frac{\pi}{2}+\epsilon,
$$

where $U=\operatorname{diag}\left[u_{1}, \ldots, u_{N}\right]$ and $\Psi=\left(\Psi_{1}, \ldots, \Psi_{N}\right)$ are as in §2.4. Moreover, we have:

(1) A fundamental solution $Y_{\tau}(z)$ satisfying this asymptotic condition is unique; we call it the asymptotically exponential fundamental solution associated to $e^{\mathrm{i} \phi}$.

(2) Let $Y_{\tau}^{-}(z)=\left(y_{1}^{-}(\tau, z), \ldots, y_{N}^{-}(\tau, z)\right)$ be the fundamental solution associated to $-e^{\mathrm{i} \phi}$. Then we have $\left(y_{i}(\tau,-z), y_{j}(\tau, z)\right)_{F}=\delta_{i j}$.

Note that the fundamental solution $Y_{\tau}(z)$ depends on the choice of sign and ordering of the normalized idempotents $\Psi_{1}, \ldots, \Psi_{N}$.

We construct $Y_{\tau}$ using Laplace transformation. Under the formal substitution $z^{-1} \rightarrow \partial_{\lambda}$, $\partial_{z^{-1}} \rightarrow-\lambda$, the differential equation $\nabla\left(z^{-1} y(\tau, z)\right)=0$ is transformed to the equations:

$$
\begin{aligned}
\widehat{\nabla}_{\alpha} \hat{y}(\tau, \lambda) & :=\left(\partial_{\alpha}-\left(\alpha \star_{\tau}\right)\left(\lambda-E \star_{\tau}\right)^{-1}\right) \hat{y}(\tau, \lambda)=0, \\
\widehat{\nabla}_{\partial_{\lambda}} \hat{y}(\tau, \lambda) & :=\left(\partial_{\lambda}+\left(\lambda-E \star_{\tau}\right)^{-1} \mu\right) \hat{y}(\tau, \lambda)=0,
\end{aligned}
$$

where $\alpha \in H^{\bullet}(F)$. See e.g. [17, Lecture 5], [54]. The connection $\widehat{\nabla}$ is flat, and has only logarithmic singularities at $\lambda=u_{1}, \ldots, u_{N}, \infty$ under the semisimplicity assumption. In fact, by the gauge transformation by $\Psi, \widehat{\nabla}$ is transformed into the following form ${ }^{4}$ :

$$
\Psi^{*} \widehat{\nabla}=d+\sum_{j=1}^{N}\left(\frac{E_{j} V}{\lambda-u_{j}}-V_{j}\right) d\left(\lambda-u_{j}\right)
$$

where $E_{j}, V, V_{j}$ are as in $\S 2.4$. This has logarithmic singularities along the normal crossing divisors $\prod_{j=1}^{N}\left(\lambda-u_{j}\right)=0$ in $B \times \mathbb{C}_{\lambda}$.

Lemma 2.5.4. Let $\left(\tau_{0}, \lambda_{0}\right) \in B \times \mathbb{C}_{\lambda}$ be a point on the singularity of $\widehat{\nabla}$.

\footnotetext{
${ }^{3}$ Our admissible direction is perpendicular to the one in [17, Definition 4.2].
}

${ }^{4}$ Here we used $\sum_{i=1}^{N} V_{i}=\Psi^{-1}\left(\partial_{u_{1}}+\cdots+\partial_{u_{N}}\right) \Psi=0$. 
(1) For every divisor $\left\{\lambda=u_{i}\right\}$ passing through $\left(\tau_{0}, \lambda_{0}\right)$, there exists a $\widehat{\nabla}$-flat section $\hat{y}_{i}(\tau, \lambda)$ which is holomorphic near $(\tau, \lambda)=\left(\tau_{0}, \lambda_{0}\right)$ and $\hat{y}_{i}\left(\tau, u_{i}(\tau)\right)=\Psi_{i}$.

(2) Let $M$ be the monodromy transformation with respect to an anti-clockwise loop around $\lambda=\lambda_{0}$ in the $\lambda$-plane. For a $\widehat{\nabla}$-flat section $\hat{y}, M \hat{y}-\hat{y}$ is a linear combination of the flat sections $\hat{y}_{i}$ in (1) such that $\left\{\lambda=u_{i}\right\}$ passes through $\left(\tau_{0}, \lambda_{0}\right)$.

Proof. (1) It suffices to find a $\Psi^{*} \widehat{\nabla}$-flat section $\hat{s}_{i}(\tau, \lambda)$ with the property $\hat{s}_{i}\left(\tau, u_{i}\right)=e_{i}$. Since we do not assume that $u_{1}, \ldots, u_{N}$ are pairwise distinct, we can have several singularity divisors passing through the point $\left(\tau_{0}, \lambda_{0}\right)$; let $\left\{\lambda=u_{j}\right\}$ be one of them. The residue $R_{j}=\left.E_{j} V\right|_{\tau=\tau_{0}}$ of $\Psi^{*} \widehat{\nabla}$ at $\left(\tau_{0}, \lambda_{0}\right)$ along the divisor $\left\{\lambda=u_{j}\right\}$ is nilpotent by Lemma 2.4.4. Thus, in a neighbourhood of $(\tau, \lambda)=\left(\tau_{0}, \lambda_{0}\right)$, we have a fundamental solution for $\widehat{\nabla}$ of the form (see, e.g. [66, Theorem 2, Remark 2]):

$$
U(\tau, \lambda) \exp \left(-\sum_{j: u_{j}\left(\tau_{0}\right)=\lambda_{0}} R_{j} \log \left(\lambda-u_{j}\right)\right)
$$

where $U(\tau, \lambda)$ is a matrix-valued holomorphic function defined near $(\tau, \lambda)=\left(\tau_{0}, \lambda_{0}\right)$ such that $U\left(\tau_{0}, \lambda_{0}\right)=$ id. We define a $\Psi^{*} \widehat{\nabla}$-flat section $\hat{s}_{i}(\tau, \lambda)$ by applying the above fundamental solution to the $i$ th basis vector $e_{i}$. Note that $R_{j} e_{i}=0$ whenever $u_{j}\left(\tau_{0}\right)=u_{i}\left(\tau_{0}\right)=\lambda_{0}$ by Lemma 2.4.4, therefore $\hat{s}_{i}(\tau, \lambda)=U(\tau, \lambda) e_{i}$ is holomorphic near $\left(\tau_{0}, \lambda_{0}\right)$. We claim that $\hat{s}_{i}\left(\tau, u_{i}\right)=e_{i}$. By definition we have $\hat{s}_{i}\left(\tau_{0}, \lambda_{0}\right)=e_{i}$. On the other hand, the residual connection $\widehat{\nabla}^{(i)}$ on the divisor $\left\{\lambda=u_{i}\right\}$ induced from $\Psi^{*} \widehat{\nabla}$ reads:

$$
\widehat{\nabla}^{(i)}=d+\sum_{j: j \neq i}\left(\frac{E_{j} V}{u_{i}-u_{j}}-V_{j}\right) d\left(u_{i}-u_{j}\right) .
$$

Using the formula (2.4.5), one finds that $E_{j} V e_{i}=\left(u_{i}-u_{j}\right) V_{j} e_{i}$ for $i \neq j$; hence $\widehat{\nabla}^{(i)} e_{i}=0$. Since $\hat{s}_{i}\left(\tau, u_{i}\right)$ is flat with respect to $\widehat{\nabla}^{(i)}$, the claim follows.

(2) This follows from the form (2.5.5) of the fundamental solution and the fact that $\operatorname{Im} R_{j} \subset$ $\mathbb{C} e_{j}$.

Proof of Proposition 2.5.1. We closely follow the method of Balser-Jurkat-Lutz [5, Theorem $2]$ and Bridgeland-Toledano-Laredo $[12, \S 8.4]$. Using the flat section $\hat{y}_{i}(\tau, \lambda)$ from Lemma 2.5.4, we define

$$
y_{i}(\tau, z)=\frac{1}{z} \int_{u_{i}+\mathbb{R}_{\geqslant 0} e^{i \phi}} \hat{y}_{i}(\tau, \lambda) e^{-\lambda / z} d \lambda .
$$

Shrinking $B$ if necessary, we may assume that $e^{-i \phi}\left(u_{i}(\tau)-u_{j}(\tau)\right) \notin \mathbb{R}$ for all $\tau \in B$ and for all $(i, j)$ with $u_{i, 0} \neq u_{j, 0}$. Then the flat section $\hat{y}_{i}(\tau, \lambda)$ can be analytically continued along the contour $u_{i}+\mathbb{R}_{\geqslant 0} e^{i \phi}$ in the $\lambda$-plane when $\tau \in B$. Indeed, by assumption, the contour $u_{i}+\mathbb{R}_{\geqslant 0} e^{\mathrm{i} \phi}$ can only contain singular points $u_{j}$ (of $\widehat{\nabla}$ ) such that $u_{i, 0}=u_{j, 0}$; but we know from Lemma 2.5.4 that $\hat{y}_{i}(\tau, \lambda)$ is holomorphic in a neighbourhood of $\left(\tau_{0}, u_{i, 0}\right)$ and thus it is regular at $\left(\tau, u_{j}\right)$ whenever $u_{i, 0}=u_{j, 0}$. Because $\widehat{\nabla}$ is regular singular at $\lambda=\infty, \hat{y}_{i}(\tau, \lambda)$ grows at most polynomially as $\lambda \rightarrow \infty$. Thus the integral (2.5.6) converges if $|\arg z-\phi|<\frac{\pi}{2}$; by changing the slope of the contour a little, we can analytically continue $y_{i}(\tau, z)$ to a bigger sector $|\arg z-\phi|<\frac{\pi}{2}+\epsilon$ with $\epsilon>0$ sufficiently small. By an elementary calculation using integration by parts, we can show that $y_{i}(\tau, z)$ is $\nabla$-flat, where we need the fact that $\hat{y}_{i}\left(\tau, u_{i}\right)$ is a $u_{i}$-eigenvector of $E \star_{\tau}$, see $[12, \S 8.4]$. Watson's lemma $[1,6.2 .2]$ shows that $y_{i}(\tau, z) \rightarrow \Psi_{i}$ as $z \rightarrow 0$ in the sector $|\arg z-\phi|<\frac{\pi}{2}+\epsilon$. 
The uniqueness of $Y_{\tau}$ follows from the fact that the angle of the sector is bigger than $\pi$ [4, Remark 1.4]. Indeed, suppose that we have two solutions $Y_{1}, Y_{2}$ subject to (2.5.2). Then there exists a constant matrix $C$ such that $Y_{1}=Y_{2} C$. We have $e^{-U / z} C e^{U / z} \rightarrow$ id as $z \rightarrow 0$ in the sector. Since the angle of the sector is bigger than $\pi$, it happens only when $C=\mathrm{id}$.

Finally we show Part (2). We omit $\tau$ from the notation. Since $y_{i}^{-}(z)$ and $y_{j}(z)$ are flat, the pairing $\left(y_{i}^{-}(-z), y_{j}(z)\right)_{F}$ does not depend on $z$ by (2.2.2). By the asymptotic condition, we have $e^{\left(u_{j}-u_{i}\right) / z}\left(y_{i}^{-}(-z), y_{j}(z)\right)_{F}=\left(e^{-u_{i} / z} y_{i}^{-}(-z), e^{u_{j} / z} y_{j}(z)\right)_{F} \rightarrow\left(\Psi_{i}, \Psi_{j}\right)_{F}=\delta_{i j}$ as $|z| \rightarrow 0$ in the sector $|\arg z-\phi|<\frac{\pi}{2}+\epsilon$. Thus $\left(y_{i}^{-}(-z), y_{j}(z)\right)_{F}=\delta_{i j}$ if $u_{i}=u_{j}$. If $u_{i} \neq u_{j}$, we have $\left(y_{i}^{-}(-z), y_{j}(z)\right)_{F}=0$ since the angle of the sector is bigger than $\pi$.

Remark 2.5.7. Applying Watson's lemma to (2.5.6), we obtain the asymptotic expansion

$$
Y_{\tau}(z) e^{U / z} \sim \Psi\left(\mathrm{id}+R_{1} z+R_{2} z^{2}+\cdots\right)
$$

as $z \rightarrow 0$ in the sector $|\arg z-\phi|<\frac{\pi}{2}+\epsilon$. The right-hand side is called a formal solution which is typically divergent. The existence of a formal solution in the case where $u_{1}, \ldots, u_{N}$ are not distinct was also remarked by Teleman [61, Theorem 8.15]. Our construction shows that each $R_{k}$ depends analytically on $\tau$, which is not clear from the standard recursive construction of a formal solution. In other words, a semisimple point of a Frobenius manifold is never a turning point.

Remark 2.5.8. Each flat section $y_{i}(\tau, z)$ in (2.5.6) can have the asymptotic expansion $y_{i}(\tau, z) \sim$ $e^{-u_{i} / z} \Psi_{i}$ in a bigger sector. Set $\Sigma_{i}=\left\{\left(u_{j}-u_{i}\right) /\left|u_{j}-u_{i}\right|: u_{j} \neq u_{i}\right\}$ and let $\left\{e^{\mathrm{i} \theta}: \xi_{1}<\theta<\xi_{2}\right\}$ be the connected component of $S^{1} \backslash \Sigma_{i}$ which contains the admissible direction $e^{i \phi}$. By construction, the flat section $y_{i}(\tau, z)$ has the asymptotic expansion in the sector $\xi_{1}-\frac{\pi}{2}<$ $\arg z<\xi_{2}+\frac{\pi}{2}$ as in Figure 1. (The sector here can be bigger than $2 \pi$.)

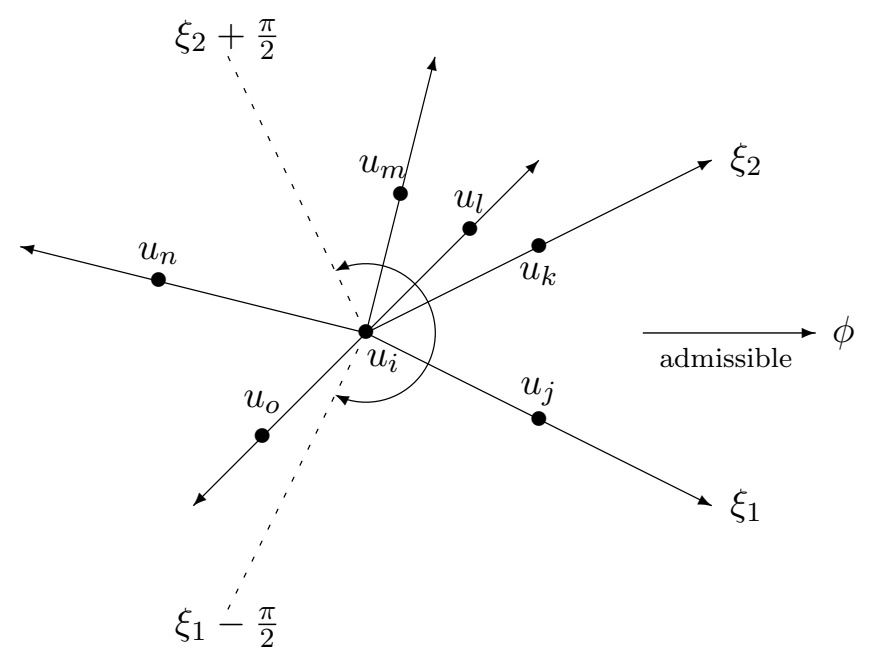

Figure 1. The flat section $y_{i}(\tau, z)$ has the asymptotics $y_{i}(\tau, z) \sim e^{-u_{i} / z} \Psi_{i}$ in the sector $\left(\xi_{1}-\frac{\pi}{2}, \xi_{2}+\frac{\pi}{2}\right)$.

2.6. Mutation and Stokes matrix. In this section, we discuss mutation of flat sections and Stokes matrices. The braid group action on the irregular monodromy data $\left(Y_{\tau}, S\right)$ via mutation was discussed by Dubrovin [17, Lecture 4]. We use the idea of Balser-Jurkat-Lutz [5] 
expressing Stokes data in terms of monodromy of the Laplace-dual connection $\widehat{\nabla}$ and extend the result of Dubrovin to the case where some of $u_{1}, \ldots, u_{N}$ may coincide. The results here lead us to the formulation of a marked reflection system in $\S 4.2-4.3, \S 4.5$.

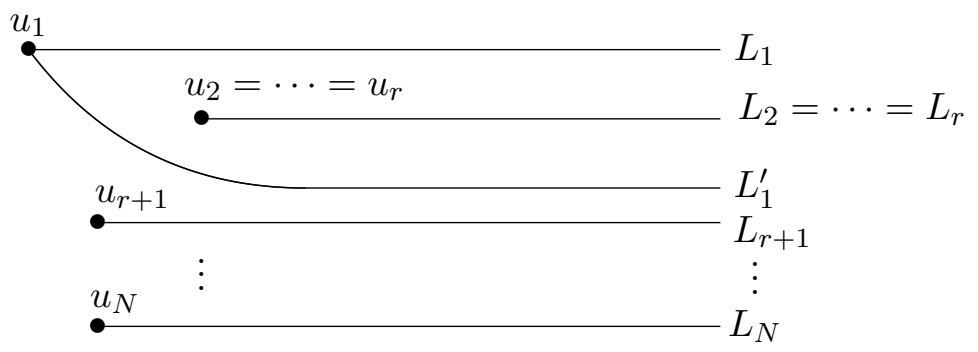

FiguRE 2. Right mutation of $L_{1}$ (where $e^{\mathrm{i} \phi}=1$ is admissible)

Recall that we constructed the asymptotically exponential flat section $y_{i}(\tau, z)$ as the Laplace transform (2.5.6) of the $\widehat{\nabla}$-flat section $\hat{y}_{i}(\tau, \lambda)$ over a straight half-line $L_{i}=u_{i}+\mathbb{R}_{\geqslant 0} e^{i \phi}$. We study the change of flat sections under a change of integration paths. To illustrate, we consider the change of paths from $L_{1}$ to $L_{1}^{\prime}$ depicted in Figure 2. In the passage from $L_{1}$ to $L_{1}^{\prime}$, the path is assumed to cross only one eigenvalue $u_{2}=\cdots=u_{r}$ of multiplicity $r-1$. We use the straight paths $L_{1}, \ldots, L_{N}$ as branch cuts for $\widehat{\nabla}$-flat sections and regard $\hat{y}_{i}(\lambda)$ as a single-valued analytic function on $\mathbb{C} \backslash \bigcup_{j \neq i} L_{j}$. Let $M$ denote the anti-clockwise monodromy transformation around $\lambda=u_{2}$ acting on the space of $\widehat{\nabla}$-flat sections. By Lemma 2.5.4 (2), the monodromy transform of $\hat{y}_{1}$ can be written as:

$$
M \hat{y}_{1}=\hat{y}_{1}-c_{12} \hat{y}_{2}-\cdots-c_{1 r} \hat{y}_{r}
$$

for some coefficients $c_{12}, \ldots, c_{1 r} \in \mathbb{C}$. From this it follows that the flat section $y_{1}^{\prime}(z)$ defined by the integral over $L_{1}^{\prime}$ is given by:

$$
y_{1}^{\prime}(z)=y_{1}(z)-c_{12} y_{2}(z)-\cdots-c_{1 r} y_{r}(z) .
$$

We call the flat section $y_{1}^{\prime}(z)$ (or the path $L_{1}^{\prime}$ ) the right mutation of $y_{1}(z)$ (resp. $L_{1}$ ) with respect to $u_{2}=\cdots=u_{r}$. The left mutation of a flat section or a path is the inverse operation: see Figure 3.

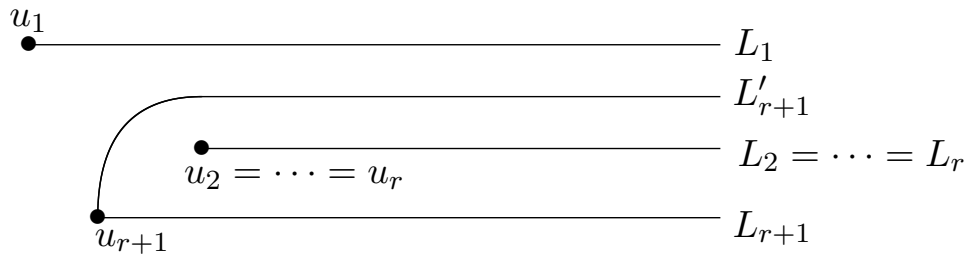

FiguRE 3. Left mutation of $L_{r+1}$

A mutation occurs when we vary the direction $e^{i \phi}$ and $e^{i \phi}$ becomes non-admissible. We now let the phase $\phi$ decrease by the angle $\pi$ continuously. Then the asymptotically exponential flat sections $y_{1}, \ldots, y_{N}$ undergo a sequence of right mutations. Let $y_{1}^{-}, \ldots, y_{N}^{-}$be the basis of asymptotically exponential flat sections associated to $-e^{i \phi}$ as in Proposition 2.5.1. In the situation of Figure 2, we successively right-mutate $L_{1}^{\prime}$ across $u_{r+1}, u_{r+2}, \ldots, u_{N}$, arriving at:

$$
y_{1}^{-}(z)=y_{1}(z)-c_{12} y_{2}(z)-\cdots-c_{1 r} y_{r}(z)-\left(\begin{array}{c}
\text { linear combinations of } y_{j}(z) \\
\text { with } \operatorname{Im}\left(e^{-\mathrm{i} \phi} u_{j}\right)<\operatorname{Im}\left(e^{-\mathrm{i} \phi} u_{2}\right)
\end{array}\right) .
$$


In this way we can write $y_{i}^{-}$as a linear combination of $y_{j}$ 's and vice versa. The transition matrix is called the Stokes matrix.

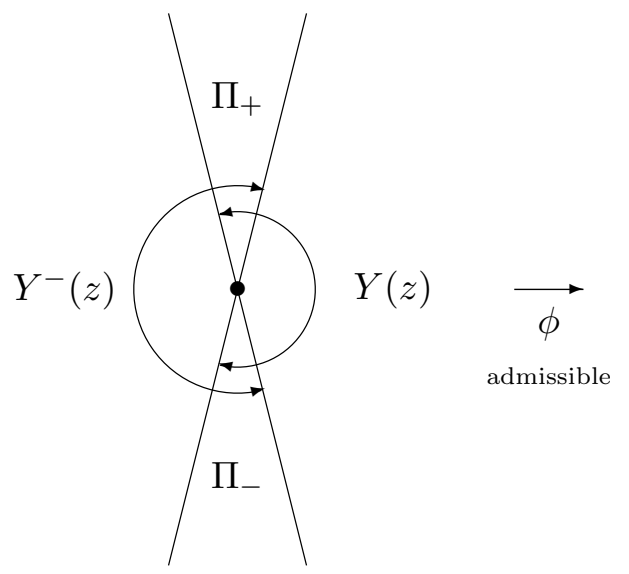

Figure 4. Domains of the two solutions $Y$ and $Y^{-}$

Definition 2.6.3 ([4, Remark 1.6], [17, Definition 4.3]). Let $Y=\left(y_{1}, \ldots, y_{N}\right), Y^{-}=$ $\left(y_{1}^{-}, \ldots, y_{N}^{-}\right)$be the fundamental solution associated to an admissible direction $e^{\mathrm{i} \phi}$ and $-e^{\mathrm{i} \phi}$ respectively as in Proposition 2.5.1. Let $\Pi_{+} \cup \Pi_{-}$be the domain in Figure 4 where both $Y$ and $Y^{-}$are defined. The Stokes matrices (or Stokes multipliers) are the constant matrices ${ }^{5}$ $S$ and $S_{-}$satisfying

$$
\begin{aligned}
& Y(z)=Y^{-}(z) S \quad \text { for } z \in \Pi_{+} \\
& Y(z)=Y^{-}(z) S_{-} \text {for } z \in \Pi_{-}
\end{aligned}
$$

Proposition 2.6.4 ([17, Theorem 4.3, (4.39)]). Let $\left(y_{1}, \ldots, y_{N}\right)$ and $\left(y_{1}^{-}, \ldots, y_{N}^{-}\right)$be the asymptotically exponential fundamental solution associated to admissible directions $e^{\mathrm{i} \phi}$ and $-e^{\mathrm{i} \phi}$ respectively and let $S=\left(S_{i j}\right), S_{-}=\left(S_{-, i j}\right)$ be the Stokes matrices. We have

(1) $S_{i j}=S_{-, j i}=\left(y_{i}\left(\tau, e^{-\pi \mathrm{i}} z\right), y_{j}(\tau, z)\right)_{F}$ for $z \in \Pi_{+}$. Here we write $e^{-\pi \mathrm{i}} z$ instead of $-z$ to specify the path of analytic continuation. Similarly, $\left(y_{i}^{-}\left(\tau, e^{\pi \mathrm{i}} z\right), y_{j}^{-}(\tau, z)\right)_{F}$ gives the coefficient $\left(S^{-1}\right)_{i j}$ of the inverse matrix of $S$.

(2) The Stokes matrix $S$ is a triangular matrix with diagonal entries all equal to one. More precisely, we have $S_{i i}=1$ for all $i$ and

$$
\begin{array}{ll}
S_{i j}=0 & \text { if } i \neq j \text { and } u_{i}=u_{j} ; \\
S_{i j}=0 & \text { if } \operatorname{Im}\left(e^{-i \phi} u_{i}\right)<\operatorname{Im}\left(e^{-i \phi} u_{j}\right) .
\end{array}
$$

In particular, $S, S_{-}$do not depend on $\tau$ and $y_{1}, \ldots, y_{N}$ are semiorthonormal.

Proof. Dubrovin [17] discussed the case where $u_{1}, \ldots, u_{N}$ are distinct. We have $y_{j}(z)=$ $\sum_{k=1}^{N} y_{k}^{-}(z) S_{k j}$ for $z \in \Pi_{+}$and $y_{j}(z)=\sum_{k=1}^{N} y_{k}^{-}(z) S_{-, k j}$ for $z \in \Pi_{-}$. Taking the pairing with $y_{i}(-z)$ and using the property $\left(y_{k}^{-}(z), y_{i}(-z)\right)_{F}=\delta_{k i}$ from Proposition 2.5.1, we obtain

$$
\begin{aligned}
& \left(y_{i}(-z), y_{j}(z)\right)_{F}=S_{i j} \quad \text { for } z \in \Pi_{+} \\
& \left(y_{i}(-z), y_{j}(z)\right)_{F}=S_{-, i j} \quad \text { for } z \in \Pi_{-}
\end{aligned}
$$

\footnotetext{
${ }^{5}$ Our Stokes matrices are inverse to the ones in [17, Definition 4.3].
} 
Replacing $z$ with $-z$ in the second formula and taking into account the direction of analytic continuation, we see that (1) and (2) hold. (The discussion for $\left(y_{i}^{-}\left(e^{\pi \dot{i}} z\right), y_{j}^{-}(z)\right)_{F}$ is similar.) As already discussed, the coefficients of the Stokes matrix arise from a sequence of mutations. Part (3) is obvious from this.

Remark 2.6.5. We often choose an ordering of normalized idempotents $\Psi_{1}, \ldots, \Psi_{N}$ so that the corresponding eigenvalues satisfy $\operatorname{Im}\left(e^{-i \phi} u_{1}\right) \geqslant \cdots \geqslant \operatorname{Im}\left(e^{-i \phi} u_{N}\right)$. Then the Stokes matrix becomes upper-triangular. Note also that flat sections $y_{i}$ corresponding to the same eigenvalue are mutually orthogonal.

Let us go back to the situation of Figure 2. The coefficient $c_{1 i}$ appearing in (2.6.2) coincides with the coefficient $S_{1 i}$ of the Stokes matrix, because by inverting (2.6.2) we obtain

$$
y_{1}(z)=y_{1}^{-}(z)+c_{12} y_{2}^{-}(z)+\cdots+c_{1 r} y_{r}^{-}(z)+\left(\begin{array}{c}
\text { linear combinations of } y_{j}^{-}(z) \\
\text { with } \operatorname{Im}\left(e^{-\mathrm{i} \phi} u_{j}\right)<\operatorname{Im}\left(e^{-\mathrm{i} \phi} u_{2}\right)
\end{array}\right)
$$

for $z \in \Pi_{-}$and therefore $c_{1 i}=S_{-, i 1}=S_{1 i}$. Recall that $c_{1 i}$ arises as the coefficient of the right mutation (2.6.1). Therefore we obtain the following corollary:

Corollary 2.6.6 ([17, Theorem 4.6]). Let $e^{\mathrm{i} \phi}$ be an admissible direction and let $\left(y_{1}, \ldots, y_{N}\right)$ be the asymptotically exponential fundamental solution associated to $e^{i \phi}$ in Proposition 2.5.1. Let $u_{a}, u_{b}$ be distinct eigenvalues such that there are no eigenvalues $u_{j}$ with $\operatorname{Im}\left(e^{-i \phi} u_{a}\right)>$ $\operatorname{Im}\left(e^{-i \phi} u_{j}\right)>\operatorname{Im}\left(e^{-i \phi} u_{b}\right)$. Then the right mutation of the flat section $y_{a}(z)$ with respect to $u_{b}$ is given by

$$
y_{a} \mapsto y_{a}-\sum_{j: u_{j}=u_{b}} S_{a j} y_{j} .
$$

Similarly, the left mutation of $y_{b}(z)$ with respect to $u_{a}$ is given by:

$$
y_{b} \mapsto y_{b}-\sum_{j: u_{j}=u_{a}} S_{j b} y_{j}
$$

where $S_{i j}=\left(y_{i}\left(\tau, e^{-\pi \mathrm{i}} z\right), y_{j}(\tau, z)\right)_{F}$ are the coefficients of the Stokes matrix.

2.7. Isomonodromic deformation. In semisimple case, the base of the big quantum connection (2.2.3) can be extended to the universal covering of a configuration space.

Suppose that the quantum product is convergent and semisimple in a neighbourhood of $\tau=0 \in H^{\bullet}(F)$. Since the eigenvalues $u_{1}, \ldots, u_{N}$ of $E \star_{\tau}$ form a local co-ordinate system $H^{\bullet}(F)$, we can make $u_{1}, \ldots, u_{N}$ pairwise distinct by a small deformation of $\tau$. We take a base point $\tau^{\circ} \in H^{\bullet}(F)$ such that the corresponding eigenvalues $\mathbf{u}^{\circ}=\left\{u_{1}^{\circ}, \ldots, u_{N}^{\circ}\right\}$ are pairwise distinct. The quantum connection $\left.\nabla\right|_{\tau^{\circ}}$ then admits a unique isomonodromic deformation over the universal cover $C_{N}(\mathbb{C}) \sim$ of the configuration space

$$
C_{N}(\mathbb{C})=\left\{\left(u_{1}, \ldots, u_{N}\right) \in \mathbb{C}^{N}: u_{i} \neq u_{j} \text { for all } i \neq j\right\} / \mathfrak{S}_{N} .
$$

Proposition 2.7.2 ([17, Lemma 3.2, Exercise 3.3, Lemma 3.3]). We have a unique meromorphic flat connection on the trivial $H^{\bullet}(F)$-bundle over $C_{N}(\mathbb{C})^{\sim} \times \mathbb{P}^{1}$ of the form:

$$
\begin{aligned}
& \nabla_{\frac{\partial}{\partial u_{i}}}=\frac{\partial}{\partial u_{i}}+\frac{1}{z} \mathcal{C}_{i} \\
& \nabla_{z \frac{\partial}{\partial z}}=z \frac{\partial}{\partial z}-\frac{1}{z} \mathcal{U}+\mu
\end{aligned}
$$

where $\mathcal{C}_{i}$ and $\mathcal{U}$ are $\operatorname{End}\left(H^{\bullet}(F)\right)$-valued holomorphic functions on $C_{N}(\mathbb{C})^{\sim}$, such that it restricts to the big quantum connection (2.2.3) in a neighbourhood of the base point $\mathbf{u}^{\circ}$. Here the eigenvalues of $\mathcal{U}$ give the co-ordinates $u_{1}, \ldots, u_{N}$ on the base. 
Remark 2.7.3 ([17, Lemma 3.3]). This isomonodromic deformation defines a Frobenius manifold structure on an open dense subset of $C_{N}(\mathbb{C})^{\sim}$.

\section{Gamma Conjecture I}

In this section we formulate Gamma conjecture I for arbitrary Fano manifolds. We do not need to assume the semisimplicity of the quantum cohomology algebra.

3.1. Property $\mathcal{O}$. We introduce Property $\mathcal{O}$ for a Fano manifold $F$.

Definition 3.1.1. Let $F$ be a Fano manifold and let $c_{1}(F) \star_{0} \in \operatorname{End}\left(H^{\bullet}(F)\right)$ be the quantum product at $\tau=0$ (see $\S 2.1)$. Set

$$
T:=\max \left\{|u|: u \text { is an eigenvalue of }\left(c_{1}(F) \star_{0}\right)\right\} .
$$

We say that $F$ satisfies Property $\mathcal{O}$ if

(1) $T$ is an eigenvalue of $\left(c_{1}(F) \star_{0}\right)$.

(2) If $u$ is an eigenvalue of $\left(c_{1}(F) \star_{0}\right)$ with $|u|=T$, we have $u=T \zeta$ for some $r$-th root of unity $\zeta \in \mathbb{C}$, where $r$ is the Fano index of $F$.

(3) The multiplicity of the eigenvalue $T$ is one.

Conjecture 3.1.2 (Conjecture $\mathcal{O}$ ). Every Fano manifold satisfies Property $\mathcal{O}$.

The number $T \in \mathbb{R}_{\geqslant 0}$ above is an algebraic number. This is an invariant of a monotone symplectic manifold. Conjecture $\mathcal{O}$ says that $T$ is non-zero unless $F$ is a point. We do not have a general argument to show Conjecture $\mathcal{O}$, but there are a few weak evidences as follows.

Remark 3.1.3. Let $r$ be the Fano index. The formula (2.2.5) shows that we have $\star_{0}=\star_{\tau}$ with $\tau=c_{1}(F)(2 \pi \mathrm{i} / r)$. This together with $(2.2 .6)$ gives the symmetry:

$$
\left(c_{1}(F) \star_{0}\right)=e^{2 \pi \mathrm{i} / r} e^{-2 \pi \mathrm{i} \mu / r}\left(c_{1}(F) \star_{0}\right) e^{2 \pi \mathrm{i} \mu / r}
$$

Therefore the spectrum of $\left(c_{1}(F) \star_{0}\right)$ is invariant under multiplication by $e^{2 \pi i / r}$.

Remark 3.1.5 ( $\mathcal{O}$ is the structure sheaf). Mirror symmetry for Fano manifolds claims that $F$ is mirror to a holomorphic function $f: Y \rightarrow \mathbb{C}$ on a complex manifold $Y$. Under mirror symmetry it is expected that the eigenvalues of $\left(c_{1}(F) \star_{0}\right)$ should coincide with the critical values of $f$. Each Morse critical point of $f$ associates a vanishing cycle (or Lefschetz thimble) which gives an object of the Fukaya-Seidel category of $f$. Under homological mirror symmetry, the vanishing cycle associated to $T$ should correspond to the structure sheaf $\mathcal{O}$ of $F$. This explains the name 'Conjecture $\mathcal{O}$ '. Our Gamma conjectures give a direct link between $T$ and $\mathcal{O}$ in terms of differential equations (without referring to mirror symmetry): the asymptotically exponential flat section associated to $T$ should correspond to $\widehat{\Gamma}_{F}=\widehat{\Gamma}_{F} \operatorname{Ch}(\mathcal{O})$; more generally a simple eigenvalue should correspond to $\widehat{\Gamma}_{F} \mathrm{Ch}(E)$ for an exceptional object $E \in \mathcal{D}_{\text {coh }}^{b}(F)$ (see Gamma Conjecture I, II in $\S 3.4, \S 4.6$ ).

Remark 3.1.6 (Conifold point [24]). Let $f:\left(\mathbb{C}^{\times}\right)^{n} \rightarrow \mathbb{C}$ be a convenient ${ }^{6}$ Laurent polynomial mirror to a Fano manifold $F$. Suppose that all the coefficients of $f$ are positive real. Then the Hessian

$$
\frac{\partial^{2} f}{\partial \log x_{i} \partial \log x_{j}}\left(x_{1}, \ldots, x_{n}\right)
$$

is positive definite on the subspace $\left(\mathbb{R}_{>0}\right)^{n}$. Thus $\left.f\right|_{\left(\mathbb{R}_{>0}\right)^{n}}$ attains a global minimum at a unique critical point $x_{\text {con }}$ in the domain $\left(\mathbb{R}_{>0}\right)^{n}$; also the critical point $x_{\text {con }}$ is non-degenerate.

\footnotetext{
${ }^{6}$ A Laurent polynomial is said to be convenient if the Newton polytope of $f$ contains the origin in its interior.
} 
We call the point $x_{\text {con }}$ the conifold point. Many examples suggest that $T$ equals $T_{\text {con }}:=$ $f\left(x_{\text {con }}\right)$.

Remark 3.1.7 (Perron-Frobenius eigenvalue). This remark is due to Kaoru Ono. We say that a square matrix $M$ of size $n$ is irreducible if the linear map $\mathbb{C}^{n} \rightarrow \mathbb{C}^{n}, v \mapsto M v$ admits no invariant co-ordinate subspace. If $\left(c_{1}(F) \star_{0}\right)$ is represented by an irreducible matrix with nonnegative entries, $T$ is a simple eigenvalue of $\left(c_{1}(F) \star_{0}\right)$ by Perron-Frobenius theorem.

Remark 3.1.8 (Fano orbifold). For a Fano orbifold, $T$ may have multiplicity bigger than one. Consider the $\mathbb{Z} / 3 \mathbb{Z}$ action on $\mathbb{P}^{2}$ given by $[x, y, z] \mapsto\left[x, e^{2 \pi \mathrm{i} / 3} y, e^{4 \pi \mathrm{i} / 3} z\right]$ and let $F$ be the quotient Fano orbifold $\mathbb{P}^{2} /(\mathbb{Z} / 3 \mathbb{Z})$. The mirror is $f=x_{1}^{-1} x_{2}^{-1}+x_{1}^{2} x_{2}^{-1}+x_{1}^{-1} x_{2}^{2}$ and $T=3$ has multiplicity three. Under homological mirror symmetry, three critical points in $f^{-1}(T)$ correspond to three flat line bundles which are mutually orthogonal in the derived category. One could guess that the multiplicity of $T$ equals the number of irreducible representations of $\pi_{1}^{\text {orb }}(F)$ for a Fano orbifold $F$.

Remark 3.1.9. In the rest of the section we assume that our Fano manifold $F$ satisfies Property $\mathcal{O}$. However, the argument in this section (i.e. $\S 3$ ) except for $\S 3.7$ works under the following weaker assumption: there exists a complex number $T$ such that (1) $T$ is an eigenvalue of $\left(c_{1}(F) \star_{0}\right)$ of multiplicity one and (2) if $\tilde{T}$ is an eigenvalue of $\left(c_{1}(F) \star_{0}\right)$ with $\operatorname{Re}(\tilde{T}) \geqslant \operatorname{Re}(T)$, then $\tilde{T}=T$. In $\S 3.7$, we use the condition part (2) in Definition 3.1.1.

When $F$ satisfies Property $\mathcal{O}$, we write

$$
T^{\prime}:=\max \left\{\operatorname{Re}(u): u \neq T, u \text { is an eigenvalue of }\left(c_{1}(F) \star_{0}\right)\right\}<T
$$

for the second biggest real part for the eigenvalues of $\left(c_{1}(F) \star_{0}\right)$.

3.2. Asymptotic solutions along positive real line. We construct a fundamental solution for $\nabla$ with nice asymptotic properties over the positive real line; here we do not assume semisimplicity, cf. $\S 2.5$.

Suppose that a Fano manifold $F$ satisfies Property $\mathcal{O}$. Let $\lambda_{1}, \ldots, \lambda_{k}$ be the distinct eigenvalues of $\left(c_{1}(F) \star_{0}\right)$ and let $N_{i}$ be the multiplicity of $\lambda_{i}$. Note that $N=N_{1}+\cdots+N_{k}$. We may assume that $T=\lambda_{1}$; Property $\mathcal{O}$ implies $N_{1}=1$. Note that the Euler vector field $E$ at $\tau=0$ equals $c_{1}(F)$. We may also assume that the matrix $U$ (2.4.1) of eigenvalues of $\left.\left(E \star_{\tau}\right)\right|_{\tau=0}=\left(c_{1}(F) \star_{0}\right)$ is of the form:

$$
U=\left(\begin{array}{cccc}
u_{1} & & & \\
& u_{2} & & \\
& & \ddots & \\
& & & u_{N}
\end{array}\right)=\left(\begin{array}{llll}
T & & & \\
& \lambda_{2} I_{N_{2}} & & \\
& & \ddots & \\
& & & \lambda_{k} I_{N_{k}}
\end{array}\right)
$$

where $I_{N_{i}}$ is the identity matrix of size $N_{i}$. Choose a linear isomorphism $\Psi: \mathbb{C}^{N} \rightarrow H^{\bullet}(F)$ which transforms $\left(c_{1}(F) \star_{0}\right)$ to the block-diagonal form:

$$
\Psi^{-1}\left(c_{1}(F) \star_{0}\right) \Psi=\left(\begin{array}{cccc}
B_{1} & & & \\
& B_{2} & & \\
& & \ddots & \\
& & & B_{k}
\end{array}\right)
$$

where $B_{i}$ is a matrix of size $N_{i}$ and $B_{i}-\lambda_{i} I_{N_{i}}$ is nilpotent. 
Proposition 3.2.1. Suppose that a Fano manifold $F$ satisfies Property $\mathcal{O}$. With the notation as above, there exists a fundamental matrix solution for the quantum connection $\nabla_{z \partial_{z}}(2.2 .1)$ at $\tau=0$ of the form

$$
P(z) e^{-U / z}\left(\begin{array}{llll}
F_{1}(z) & & & \\
& F_{2}(z) & & \\
& & \ddots & \\
& & & F_{k}(z)
\end{array}\right)
$$

over a sufficiently small sector $\mathcal{S}=\left\{z \in \mathbb{C}^{\times}:|z| \leqslant 1,|\arg (z)| \leqslant \epsilon\right\}$ with $\epsilon>0$ such that

(1) $P(z)$ has an asymptotic expansion $P(z) \sim \Psi+P_{1} z+P_{2} z^{2}+\cdots$ as $z \rightarrow 0$ in $\mathcal{S}$;

(2) $F_{i}(z)$ is a $G L_{N_{i}}(\mathbb{C})$-valued function satisfying the estimate $\max \left(\left\|F_{i}(z)\right\|,\left\|F_{i}(z)^{-1}\right\|\right) \leqslant$ $C \exp \left(\delta|z|^{-p}\right)$ on $\mathcal{S}$ for some $C, \delta>0$ and $0<p<1$.

(3) $F_{1}(z)=1$.

Proof. By a result of Sibuya [58] and Wasow [65, Theorem 12.2], we can find a gauge transformation $P(z)$ over $\mathcal{S}$ satisfying (1) above such that the new connection matrix $C(z)$

$$
P(z)^{-1} \nabla_{\partial_{z}} P(z)=\partial_{z}-z^{-2} C(z)
$$

satisfies:

- $C(z)=\operatorname{diag}\left[C_{1}(z), \ldots, C_{k}(z)\right]$ is block-diagonal with $C_{i}(z)$ being of size $N_{i}$;

- $C_{i}(z)$ has an asymptotic expansion $C_{i}(z) \sim C_{i, 0}+C_{i, 1} z+C_{i, 2} z^{2}+\cdots$ as $z \rightarrow 0$ in $\mathcal{S}$;

- $C_{i, 0}-\lambda_{i} I_{N_{i}}$ is nilpotent.

Now it suffices to find a fundamental solution for the $i$ th block $\nabla^{(i)}:=\partial_{z}-z^{-2} C_{i}(z)$. We have $e^{\lambda_{i} / z} \nabla^{(i)} e^{-\lambda_{i} / z}=\partial_{z}-z^{-2}\left(C_{i}(z)-\lambda_{i} I_{N_{i}}\right)$ and the leading term of the asymptotic expansion of $C_{i}(z)-\lambda_{i} I_{N_{i}}$ is nilpotent. By [59, Lemma 5.4.1], any fundamental solution $F_{i}(z)$ for $\partial_{z}-z^{-2}\left(C_{i}(z)-\lambda_{i} I_{N_{i}}\right)$ satisfies the estimate in part (2) for $1-1 / N_{i}<p<1$.

We show that part (3) can be achieved. Set $D(z)=C_{1}(z)-\lambda_{1}$. Then $F_{1}(z)$ is a solution to the scalar differential equation $\left(\partial_{z}-z^{-2} D(z)\right) F_{1}(z)=0$. Arguing as in [65, Theorem 12.3], we find that $F_{1}(z)=z^{c} g(z)$ for some $c \in \mathbb{C}$ and a holomorphic function $g(z)$ on $\mathcal{S}$ which has an asymptotic expansion $g(z) \sim a_{0}+a_{1} z+a_{2} z^{2}+\cdots$ at $z=0$ with $a_{0} \neq 0$. Thus we obtain a $\nabla$-flat section $s_{1}(z)=e^{-T / z} z^{c} g(z) P_{1}(z)$ where $P_{1}(z)$ is the first column of $P(z)$. Studying the asymptotics of the equation $\nabla s_{1}(z)=0$ (using e.g. [65, Theorem 8.8]), we have

$$
(\mu+c) a_{0} P_{1}(0) \in \operatorname{Im}\left(\left(c_{1}(F) \star_{0}\right)-T\right) .
$$

Recall that $P_{1}(0)$ is a non-zero $T$-eigenvector of $\left(c_{1}(F) \star_{0}\right)$. By Lemma 3.2.2 below, we must have $c=0$. Thus we can absorb $F_{1}(z)=g(z)$ into the first column of $P(z)$.

Lemma 3.2.2. Suppose that a Fano manifold $F$ satisfies Property $\mathcal{O}$. Let $E(T) \subset H^{\bullet}(F)$ denote the one-dimensional $T$-eigenspace of $\left(c_{1}(F) \star_{0}\right)$ and define $H^{\prime}:=\operatorname{Im}\left(T-\left(c_{1}(F) \star_{0}\right)\right)$ which is a complementary subspace of $E(T)$. Then $E(T)$ and $H^{\prime}$ are orthogonal with respect to the Poincaré pairing and the endomorphism $\mu$ satisfies $\mu(E(T)) \subset H^{\prime}$.

Proof. Take $\alpha \in E(T)$ and $\beta \in H^{\prime}$. There exists an element $\gamma \in H^{\bullet}(F)$ such that $\beta=$ $\left(T-c_{1}(F) \star_{0}\right) \gamma$. Then we have

$$
(\alpha, \beta)_{F}=\left(\alpha, T \gamma-c_{1}(F) \star_{0} \gamma\right)_{F}=\left(T \alpha-c_{1}(F) \star_{0} \alpha, \gamma\right)_{F}=0
$$

Thus $E(T) \perp H^{\prime}$ with respect to $(\cdot, \cdot)_{F}$. The skew-adjointness of $\mu$ with respect to $(\cdot, \cdot)_{F}$ shows that $(\mu \alpha, \alpha)_{F}=0$; hence $\mu(\alpha) \in H^{\prime}$. 
3.3. Flat sections with the smallest asymptotics. Under Property $\mathcal{O}$, one can find a one-dimensional vector space $\mathcal{A}$ of flat sections $s(z)$ having the most rapid exponential decay $s(z) \sim e^{-T / z}$ as $z \rightarrow+0$ on the positive real line. We refer to such sections as flat sections with the smallest asymptotics. It is easy to deduce the following result from Proposition 3.2.1.

Proposition 3.3.1. Suppose that a Fano manifold $F$ satisfies Property $\mathcal{O}$. Consider the quantum connection $\nabla$ in (2.2.1) and define:

$$
\mathcal{A}:=\left\{s: \mathbb{R}_{>0} \rightarrow H^{\bullet}(F): \nabla s=0,\left\|e^{T / z} s(z)\right\|=O\left(z^{-m}\right) \text { as } z \rightarrow+0(\exists m)\right\}
$$

Then:

(1) $\mathcal{A}$ is a one-dimensional complex vector space.

(2) For $s \in \mathcal{A}$, the limit $\lim _{z \rightarrow+0} e^{T / z} s(z)$ exists and lies in the $T$-eigenspace $E(T)$ of $\left(c_{1}(F) \star_{0}\right)$. The map $\mathcal{A} \rightarrow E(T)$ defined by this limit is an isomorphism.

(3) Let $s: \mathbb{R}_{>0} \rightarrow H^{\bullet}(F)$ be a flat section which does not belong to $\mathcal{A}$. Then we have $\lim _{z \rightarrow+0}\left\|e^{\lambda / z} s(z)\right\|=\infty$ for all $\lambda>T^{\prime}$ where $T^{\prime}$ is in (3.1.10).

Proof. With the notation as in Proposition 3.2.1, any $\nabla$-flat section $s(z)$ on the positive real line can be written in the form:

$$
s(z)=P(z) e^{-U / z}\left(F_{1}(z) v_{1}+\cdots+F_{k}(z) v_{k}\right)
$$

with $v_{i} \in \mathbb{C}^{N_{i}}$ constant vectors. We claim that $s(z)$ belongs to $\mathcal{A}$ if and only if $v_{2}=v_{3}=\cdots=$ $v_{k}=0$. The 'if' part of the claim is obvious from Proposition 3.2.1. Suppose that $s(z) \in \mathcal{A}$. Take $i \geqslant 2$. The assumption gives that $e^{\left(T-\lambda_{i}\right) / z} F_{i}(z) v_{i}$ is of polynomial growth as $z \rightarrow+0$. (The factor $P(z)$ is irrelevant, as it converges to the invertible matrix $\Psi$ as $z \rightarrow+0$.) On the other hand, from $\operatorname{Re}\left(\lambda_{i}\right)<T$ and the estimate of the norm $\left\|F_{i}(z)^{-1}\right\|$ in Proposition 3.2.1, we obtain an estimate of the form $\left\|e^{\left(\lambda_{i}-T\right) / z} F_{i}(z)^{-1}\right\| \leqslant C e^{-\varepsilon / z}$ on $|z| \leqslant 1$ for some $C, \varepsilon>0$. By taking the limit $z \rightarrow+0$ in the inequality

$$
\left\|v_{i}\right\| \leqslant\left\|e^{\left(\lambda_{i}-T\right) / z} F_{i}(z)^{-1}\right\|\left\|e^{\left(T-\lambda_{i}\right) / z} F_{i}(z) v_{i}\right\| \leqslant C e^{-\varepsilon / z}\left\|e^{\left(T-\lambda_{i}\right) / z} F_{i}(z) v_{i}\right\|,
$$

we find that $v_{i}=0$. The claim follows. Parts (1) and (2) of the proposition follows easily from the claim. To show part (3), it suffices to show that $\left\|e^{\left(\lambda-\lambda_{i}\right) / z} F_{i}(z) v_{i}\right\| \rightarrow \infty$ as $z \rightarrow+0$ for $i \geqslant 2, \lambda>T^{\prime}$ and $v_{i} \neq 0$. Using again the estimate for $\left\|F_{i}(z)^{-1}\right\|$, we have an estimate of the form $\left\|e^{\left(\lambda_{i}-\lambda\right) / z} F_{i}(z)^{-1}\right\| \leqslant C e^{-\varepsilon / z}$ on $|z| \leqslant 1$ for some $C, \varepsilon>0$. The inequality

$$
0<\left\|v_{i}\right\| \leqslant\left\|e^{\left(\lambda_{i}-\lambda\right) / z} F_{i}(z)^{-1}\right\|\left\|e^{\left(\lambda-\lambda_{i}\right) / z} F_{i}(z) v_{i}\right\|
$$

now implies that $\lim _{z \rightarrow+0}\left\|e^{\left(\lambda-\lambda_{i}\right) / z} F_{i}(z) v_{i}\right\|=\infty$.

3.4. Gamma Conjecture I: statement. The Gamma class [51, 52, 44, 49, 45] of a smooth projective variety $F$ is the class

$$
\widehat{\Gamma}_{F}:=\prod_{i=1}^{\operatorname{dim} F} \Gamma\left(1+\delta_{i}\right) \in H^{\bullet}(F)
$$

where $\delta_{1}, \ldots, \delta_{\operatorname{dim} F}$ are the Chern roots of the tangent bundle $T F$ so that $c(T F)=\prod_{i=1}^{\operatorname{dim} F}\left(1+\delta_{i}\right)$. The Gamma function $\Gamma\left(1+\delta_{i}\right)$ in the right-hand side should be expanded in the Taylor series in $\delta_{i}$. We have

$$
\widehat{\Gamma}_{F}=\exp \left(-C_{\mathrm{eu}} c_{1}(F)+\sum_{k=2}^{\infty}(-1)^{k}(k-1) ! \zeta(k) \operatorname{ch}_{k}(T F)\right)
$$


where $C_{\text {eu }}=0.57721 \ldots$ is Euler's constant and $\zeta(k)$ is the special value of Riemann's zeta function.

The fundamental solution $S(z) z^{-\mu} z^{\rho}$ from Proposition 2.3.1 identifies the space of flat sections over the positive real line $\mathbb{R}_{>0}$ with the cohomology group:

$$
\begin{aligned}
\Phi: H^{\bullet}(F) & \longrightarrow\left\{s: \mathbb{R}_{>0} \rightarrow H^{\bullet}(F): \nabla s=0\right\} \\
\alpha & \longmapsto(2 \pi)^{-\frac{\operatorname{dim} F}{2}} S(z) z^{-\mu} z^{\rho} \alpha
\end{aligned}
$$

where we use the standard determination for $z^{-\mu} z^{\rho}=\exp (-\mu \log z) \exp (\rho \log z)$ such that $\log z \in \mathbb{R}$ for $z \in \mathbb{R}_{>0}$.

Definition 3.4.2. Suppose that a Fano manifold $F$ satisfies Property $\mathcal{O}$. The principal asymptotic class of $F$ is a cohomology class $A_{F} \in H^{*}(F)$ such that $\mathcal{A}=\mathbb{C} \Phi\left(A_{F}\right)$, where $\mathcal{A}$ is the space (3.3.2) of flat sections with the smallest asymptotics (recall that $\mathcal{A}$ is of dimension one by Proposition 3.3.1). The class $A_{F}$ is defined up to a constant; when $\left\langle[\mathrm{pt}], A_{F}\right\rangle \neq 0$, we can normalize $A_{F}$ so that $\left\langle[\mathrm{pt}], A_{F}\right\rangle=1$.

Conjecture 3.4.3 (Gamma Conjecture I). Let $F$ be a Fano manifold $F$ satisfying Property $\mathcal{O}$. The principal asymptotic class $A_{F}$ of $F$ is given by $\widehat{\Gamma}_{F}$.

We explain that the Gamma class should be viewed as a square root of the Todd class (or $\widehat{A}$-class) in the Riemann-Roch theorem. Let $H$ denote the vector space of $\nabla$-flat sections over the positive real line:

$$
H:=\left\{s: \mathbb{R}_{>0} \rightarrow H^{\bullet}(F): \nabla s=0\right\}
$$

and let $[\cdot, \cdot)$ be a non-symmetric pairing on $H$ defined by

$$
\left[s_{1}, s_{2}\right):=\left(s_{1}\left(e^{-i \pi} z\right), s_{2}(z)\right)_{F}
$$

for $s_{1}, s_{2} \in H$, where $s_{1}\left(e^{-\mathrm{i} \pi} z\right)$ denotes the analytic continuation of $s_{1}(z)$ along the semicircle $[0,1] \ni \theta \mapsto e^{-i \pi \theta} z$. The right-hand side does not depend on $z$ because of the flatness (2.2.2). The non-symmetric pairing $[\cdot, \cdot)$ induces a pairing on $H^{\bullet}(F)$ via the map $\Phi(3.4 .1)$; we denote by the same symbol $[\cdot, \cdot)$ the corresponding pairing on $H^{*}(F)$. Since $S(z)$ preserves the pairing (Proposition 2.3.1), we have

$$
[\alpha, \beta)=\frac{1}{(2 \pi)^{\operatorname{dim} F}}\left(e^{\pi \mathbf{i} \mu} e^{-\pi \dot{\mathbf{i}} \rho} \alpha, \beta\right)_{F}=\frac{1}{(2 \pi)^{\operatorname{dim} F}}\left(e^{\pi \mathbf{i} \rho} e^{\pi \dot{\mathrm{i}} \mu} \alpha, \beta\right)_{F}
$$

for $\alpha, \beta \in H^{\bullet}(F)$. We write $\mathrm{Ch}(\cdot), \operatorname{Td}(\cdot)$ for the following characteristic classes:

$$
\begin{aligned}
& \operatorname{Ch}(V)=(2 \pi \mathrm{i})^{\frac{\operatorname{deg}}{2}} \operatorname{ch}(V)=\sum_{j=1}^{\operatorname{rank} V} e^{2 \pi \mathrm{i} \delta_{j}} \\
& \operatorname{Td}(V)=(2 \pi \mathrm{i})^{\frac{\operatorname{deg}}{2}} \operatorname{td}(V)=\prod_{j=1}^{\operatorname{rank} V} \frac{2 \pi \mathrm{i} \delta_{j}}{1-e^{-2 \pi \mathrm{i} \delta_{j}}}
\end{aligned}
$$

where $\delta_{1}, \ldots, \delta_{\operatorname{rank} V}$ are the Chern roots of a vector bundle $V$. We also write $\operatorname{Td}_{F}=\operatorname{Td}(T F)$.

Lemma 3.4.6 $([44,45])$. Let $V_{1}, V_{2}$ be vector bundles over $F$. Then

$$
\left[\widehat{\Gamma}_{F} \operatorname{Ch}\left(V_{1}\right), \widehat{\Gamma}_{F} \operatorname{Ch}\left(V_{2}\right)\right)=\chi\left(V_{1}, V_{2}\right)
$$

where $\chi\left(V_{1}, V_{2}\right)=\sum_{i=0}^{\operatorname{dim} F}(-1)^{i} \operatorname{dim} \operatorname{Ext}^{i}\left(V_{1}, V_{2}\right)$. 
Proof. Recall the Gamma function identity:

$$
\Gamma(1+z) \Gamma(1-z)=\frac{2 \pi \mathrm{i} z}{e^{\mathrm{i} \pi z}-e^{-\mathbf{i} \pi z}} .
$$

This immediately implies that

$$
e^{\pi \mathrm{i} \rho}\left(e^{\pi \mathrm{i} \frac{\operatorname{deg}}{2}} \widehat{\Gamma}_{F}\right) \cdot \widehat{\Gamma}_{F}=\operatorname{Td}_{F}
$$

Therefore

$$
\left[\widehat{\Gamma}_{F} \operatorname{Ch}\left(V_{1}\right), \widehat{\Gamma}_{F} \operatorname{Ch}\left(V_{2}\right)\right)=\frac{1}{(2 \pi \mathrm{i})^{\operatorname{dim} F}} \int_{F}\left(e^{\pi \mathrm{i} \frac{\mathrm{deg}}{2}} \operatorname{Ch}\left(V_{1}\right)\right) \operatorname{Ch}\left(V_{2}\right) \operatorname{Td}_{F} .
$$

This equals $\chi\left(V_{1}, V_{2}\right)$ by the Hirzebruch-Riemann-Roch formula.

Under Gamma Conjecture I, the canonical generator $\Phi\left(\widehat{\Gamma}_{F}\right)$ of $\mathcal{A}$ satisfies the following normalization.

Proposition 3.4.8. Suppose that a Fano manifold $F$ satisfies Property $\mathcal{O}$ and Gamma Conjecture I. Set $s_{1}(z)=\Phi\left(\widehat{\Gamma}_{F}\right)(z)$. Then we have:

(1) The limit $v:=\lim _{z \rightarrow+0} e^{T / z} s_{1}(z) \in E(T)$ satisfies $(v, v)_{F}=1$.

(2) Let $\widehat{\nabla}_{\partial_{\lambda}}$ be the Laplace dual (2.5.3) of the quantum connection $\nabla$ at $\tau=0$. There exists a $\widehat{\nabla}_{\partial_{\lambda}}$ flat section $\varphi(\lambda)$ which is holomorphic near $\lambda=T$ such that $\varphi(T)=v$ and that $s_{1}(z)=z^{-1} \int_{T}^{\infty} \varphi(\lambda) e^{-\lambda / z} d \lambda$.

Proof. We give a construction of the generator $s_{1}$ of $\mathcal{A}$ using Laplace transformation as in $\S 2.5$. Recall that once we have a $\widehat{\nabla}$-flat section $\varphi(\lambda)$ holomorphic near $\lambda=T$ such that $\varphi(T)$ is a non-zero eigenvector $v$ of $\left(c_{1}(F) \star_{0}\right)$ with eigenvalue $T$, the Laplace transform

$$
s_{1}(z)=\frac{1}{z} \int_{T}^{\infty} \varphi(\lambda) e^{-\lambda / z} d \lambda
$$

gives a $\nabla$-flat section such that $\lim _{z \rightarrow+0} e^{T / z} s_{1}(z)=v$ (see the discussion around (2.5.6)). Thus it suffices to construct a $\widehat{\nabla}$-flat section $\varphi(\lambda)$ as above. Property $\mathcal{O}$ ensures that $\widehat{\nabla}$ is logarithmic at $\lambda=T$, and Lemma 3.2.2 ensures that the residue $R$ of $\widehat{\nabla}$ at $\lambda=T$ is nilpotent and $R v=0$. Thus we have a fundamental matrix solution for $\widehat{\nabla}$ of the form $U(\lambda) \exp (-R \log (\lambda-T))$ with $U(\lambda)$ holomorphic near $\lambda=T$ and $U(T)=$ id $[65$, Theorem 5.5]. Now $\varphi(\lambda):=U(\lambda) \exp (-R \log (\lambda-T)) v=U(\lambda) v$ gives the desired $\widehat{\nabla}$-flat section.

It now suffices to show that $v=\varphi(T)$ is of unit length. By bending the integration path $[T, \infty]$ as in Figure 5, we obtain the analytic continuation $s_{1}\left(e^{-\pi \mathrm{i}} z\right)$ of $s_{1}(z)$. Watson's lemma $[1, \S 6.2 .2]$ shows that $\lim _{z \rightarrow+0} e^{-T / z} s_{1}\left(e^{-\pi \mathrm{i}} z\right)=v$. Therefore we have

$$
\left[\widehat{\Gamma}_{F}, \widehat{\Gamma}_{F}\right)=\left(\Phi\left(\widehat{\Gamma}_{F}\right)\left(e^{-\pi \mathrm{i}} z\right), \Phi\left(\widehat{\Gamma}_{F}\right)(z)\right)_{F}=\left(s_{1}\left(e^{-\pi \mathrm{i}} z\right), s_{1}(z)\right)_{F} \rightarrow(v, v)_{F}
$$

as $z \rightarrow+0$. By Lemma 3.4.6, the left-hand side equals $\chi\left(\mathcal{O}_{F}, \mathcal{O}_{F}\right)=1$ since $F$ is Fano. Thus we have $(v, v)_{F}=1$ and the conclusion follows. 


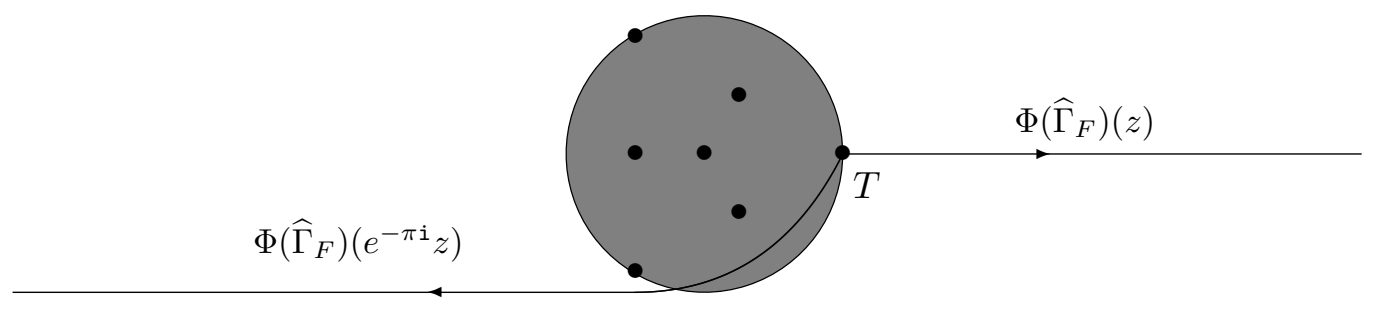

FiguRE 5 . Spectrum of $\left(c_{1}(F) \star_{0}\right)$ and the paths of integration (on the $\lambda$-plane).

3.5. The leading asymptotics of dual flat sections. We now study flat sections of the dual quantum connection, which we call dual flat sections. We show that the limit $\lim _{z \rightarrow+0} e^{T / z} f(z)$ exists for every dual flat section $f(z)$.

Let $H_{.}(F)=H_{\text {even }}(F ; \mathbb{C})$ denote the even part of the homology group with complex coefficients. The dual quantum connection is a meromorphic flat connection on the trivial bundle $H .(F) \times \mathbb{P}^{1} \rightarrow \mathbb{P}^{1}$ given by (cf. (2.2.1))

$$
\nabla_{z \frac{\partial}{\partial z}}^{\vee}=z \frac{\partial}{\partial z}+\frac{1}{z}\left(c_{1}(F) \star_{0}\right)^{\mathrm{t}}-\mu^{\mathrm{t}}
$$

where the superscript "t" denotes the transpose. This is dual to the quantum connection $\nabla$ in the sense that we have

$$
d\langle f(z), s(z)\rangle=\left\langle\nabla^{\vee} f(z), s(z)\right\rangle+\langle f(z), \nabla s(z)\rangle
$$

where $\langle\cdot, \cdot\rangle$ is the natural pairing between homology and cohomology. Because of the property (2.2.2), we can identify the dual flat connection $\nabla^{\vee}$ with the quantum connection $\nabla$ with the opposite sign of $z$. In order to avoid possible confusion about signs, we shall not use this identification. We write $E(T)^{*} \subset H_{\bullet}(F)$ for the one-dimensional eigenspace of $\left(c_{1}(F) \star_{0}\right)^{\mathrm{t}}$ with eigenvalue $T$ and $H^{\prime *}=\operatorname{Im}\left(T-\left(c_{1}(F) \star_{0}\right)^{\mathrm{t}}\right)$ for a complementary subspace. They are dual to $E(T), H^{\prime}$ from Lemma 3.2.2.

Proposition 3.5.2. Suppose that a Fano manifold $F$ satisfies Property $\mathcal{O}$. Then:

(1) The limit

$$
\operatorname{Lead}(f):=\lim _{z \rightarrow+0} e^{-T / z} f(z)
$$

exists for every $\nabla^{\vee}$-flat section $f(z)$ and lies in $E(T)^{*}$. In other words, Lead defines a linear functional:

$$
\text { Lead }:\left\{f: \mathbb{R}_{>0} \rightarrow H_{\bullet}(F): \nabla^{\vee} f(z)=0\right\} \longrightarrow E(T)^{*} .
$$

(2) If $\operatorname{Lead}(f)=0$, we have $\lim _{z \rightarrow+0} e^{-\lambda / z} f(z)=0$ for every $\lambda>T^{\prime}$.

Proof. Note that a fundamental solution for $\nabla^{\vee}$ is given by the inverse transpose of that for $\nabla$. The conclusion follows easily by using the fundamental solution in Proposition 3.2.1.

3.6. A limit formula for the principal asymptotic class. The leading asymptotic behaviour of dual flat sections as $z \rightarrow+0$ is given by the pairing with a flat section with the smallest asymptotics. From this we obtain a limit formula for $A_{F}$. 
The fundamental solution for the dual quantum connection $\nabla^{\vee}$ (3.5.1) is given by the inverse transpose $\left(S(z) z^{-\mu} z^{\rho}\right)^{-\mathrm{t}}$ of the fundamental solution for $\nabla$ in Proposition 2.3.1. This yields the following identification (cf. (3.4.1))

$$
\begin{gathered}
\Phi^{\vee}: H_{\bullet}(F) \longrightarrow\left\{f: \mathbb{R}_{>0} \rightarrow H_{\bullet}(F): \nabla^{\vee} f(z)=0\right\} \\
\alpha \longmapsto(2 \pi)^{\frac{\operatorname{dim} F}{2}} S(z)^{-\mathrm{t}} z^{\mu^{\mathrm{t}}} z^{-\rho^{\mathrm{t}}} \alpha
\end{gathered}
$$

between dual flat sections and homology classes.

Proposition 3.6.2. Suppose that $F$ satisfies Property $\mathcal{O}$. Let $A_{F}$ be a principal asymptotic class (Definition 3.4.2). Let $\psi_{0}^{*}$ be an element of $E(T)^{*}$ which is dual to $\psi_{0}:=$ $\lim _{z \rightarrow+0} e^{T / z} \Phi\left(A_{F}\right)(z) \in E(T)$ in the sense that $\left\langle\psi_{0}^{*}, \psi_{0}\right\rangle=1$. Then we have:

$$
\operatorname{Lead}\left(\Phi^{\vee}(\alpha)\right):=\lim _{z \rightarrow+0} e^{-T / z} \Phi^{\vee}(\alpha)(z)=\left\langle\alpha, A_{F}\right\rangle \psi_{0}^{*} .
$$

Proof. By the definition of $\Phi$ and $\Phi^{\vee}$ (see (3.4.1), (3.6.1)), we have:

$$
\left\langle\alpha, A_{F}\right\rangle=\left\langle\Phi^{\vee}(\alpha)(z), \Phi\left(A_{F}\right)(z)\right\rangle=\left\langle e^{-T / z} \Phi^{\vee}(\alpha)(z), e^{T / z} \Phi\left(A_{F}\right)(z)\right\rangle .
$$

This converges to $\left\langle\operatorname{Lead}\left(\Phi^{\vee}(\alpha)\right), \psi_{0}\right\rangle$ as $z \rightarrow+0$ by Propositions 3.3.1, 3.5.2.

Definition 3.6.3 ([29]). Let $S(z) z^{-\mu} z^{\rho}$ be the fundamental solution from Proposition 2.3.1 and set $t:=z^{-1}$. The $J$-function $J(t)$ of $F$ is a cohomology-valued function defined by:

$$
J(t):=z^{\frac{\operatorname{dim} F}{2}} z^{-\rho} z^{\mu} S(z)^{-1} 1
$$

where $1 \in H^{\bullet}(F)$ is the identity class. Alternatively we can define $J(t)$ by the requirement that

$$
\langle\alpha, J(t)\rangle=\left(\frac{z}{2 \pi}\right)^{\frac{\operatorname{dim} F}{2}}\left\langle\Phi^{\vee}(\alpha)(z), 1\right\rangle
$$

holds for all $\alpha \in H_{\bullet}(F)$, using the dual flat sections $\Phi^{\vee}(\alpha)$ in (3.6.1).

Remark 3.6.5. The $J$-function is a solution to the scalar differential equation associated to the quantum connection on the anticanonical line $\tau=c_{1}(F) \log t$. More precisely, we have

$$
P\left(t,\left[\nabla_{c_{1}(F)}\right]_{z=1}\right) 1=0 \Longleftrightarrow P\left(t, t \frac{\partial}{\partial t}\right) J(t)=0
$$

for any differential operators $P \in \mathbb{C}\left\langle t, t \frac{\partial}{\partial t}\right\rangle$, where $\left[\nabla_{c_{1}(F)}\right]_{z=1}=t \frac{\partial}{\partial t}+\left(c_{1}(F) \star_{c_{1}(F) \log t}\right)$. Differential equations satisfied by the $J$-function are called the quantum differential equations. See also Remark 2.2.4.

Remark 3.6.6. Using the fact that $S(z)^{-1}$ equals the adjoint of $S(-z)$ (Proposition 2.3.1) and Remark 2.3.2, we have

$$
J(t)=e^{c_{1}(F) \log t}\left(1+\sum_{i=1}^{N} \sum_{d \in \operatorname{Eff}(F) \backslash\{0\}}\left\langle\frac{\phi_{i}}{1-\psi}\right\rangle_{0,1, d}^{F} t^{c_{1}(F) \cdot d} \phi^{i}\right)
$$

where $\left\{\phi_{i}\right\}_{i=1}^{N}$ and $\left\{\phi^{i}\right\}_{i=1}^{N}$ are bases of $H^{\bullet}(F)$ which are dual with respect to the Poincaré pairing $(\cdot, \cdot)_{F}$. This gives a well-known form of Givental's $J$-function [29] restricted to the anticanonical line $\tau=c_{1}(F) \log t$.

Theorem 3.6.8. Suppose that a Fano manifold $F$ satisfies Property $\mathcal{O}$. Let $A_{F}$ be a principal asymptotic class (Definition 3.4.2) and let $J(t)$ be the $J$-function. We have

$$
\lim _{t \rightarrow+\infty} \frac{J(t)}{\langle[\mathrm{pt}], J(t)\rangle}=\frac{A_{F}}{\left\langle[\mathrm{pt}], A_{F}\right\rangle}
$$


where the limit is taken over the positive real line and exists if and only if $\left\langle[\mathrm{pt}], A_{F}\right\rangle \neq 0$.

Proof. By Proposition 3.6.2, we have

$$
\begin{aligned}
\lim _{z \rightarrow+0} e^{-T / z} \Phi^{\vee}(\alpha)(z) & =\left\langle\alpha, A_{F}\right\rangle \psi_{0}^{*} \\
\lim _{z \rightarrow+0} e^{-T / z} \Phi^{\vee}([\mathrm{pt}])(z) & =\left\langle[\mathrm{pt}], A_{F}\right\rangle \psi_{0}^{*}
\end{aligned}
$$

for some non-zero $\psi_{0}^{*} \in E(T)^{*}$. Therefore by (3.6.4) we have

$$
\lim _{t \rightarrow+\infty} \frac{\langle\alpha, J(t)\rangle}{\langle[\mathrm{pt}], J(t)\rangle}=\lim _{z \rightarrow+0} \frac{e^{-T / z}\left\langle\Phi^{\vee}(\alpha)(z), 1\right\rangle}{e^{-T / z}\left\langle\Phi^{\vee}([\mathrm{pt}])(z), 1\right\rangle}=\frac{\left\langle\alpha, A_{F}\right\rangle}{\left\langle[\mathrm{pt}], A_{F}\right\rangle}
$$

for all $\alpha \in H_{.}(F)$. Here we used the fact $\left\langle\psi_{0}^{*}, 1\right\rangle \neq 0$ which is proved in Lemma 3.6.10 below. The limit exists if and only if $\left\langle[\mathrm{pt}], A_{F}\right\rangle \neq 0$. The Theorem is proved.

Corollary 3.6.9. Suppose that a Fano manifold $F$ satisfies Property $\mathcal{O}$. The following statements are equivalent:

(1) Gamma Conjecture I (Conjecture 3.4.3) holds for $F$.

(2) $\left\|\Phi\left(\widehat{\Gamma}_{F}\right)(z)\right\| \leqslant C e^{-\lambda / z}$ on $(0,1]$ for some $C>0$ and $\lambda>T^{\prime}$, where $T^{\prime}$ is given in (3.1.10) and $\Phi$ is given in (3.4.1).

(3) We have

$$
\lim _{t \rightarrow+\infty} \frac{J(t)}{\langle[\mathrm{pt}], J(t)\rangle}=\widehat{\Gamma}_{F}
$$

where $J(t)$ is the $J$-function of $F$ given in Definition 3.6.3 and the limit is taken over the positive real line.

Proof. The equivalence of (1) and (2) is clear from Proposition 3.3.1. The equivalence of (1) and (3) follows from Theorem 3.6.8.

Lemma 3.6.10. Suppose that $F$ satisfies Property $\mathcal{O}$. For a non-zero eigenvector $\psi_{0}^{*} \in$ $E(T)^{*}$, we have $\left\langle\psi_{0}^{*}, 1\right\rangle \neq 0$. In other words, the $E(T)$-component of 1 with respect to the decomposition $H^{\bullet}(F)=E(T) \oplus H^{\prime}$ in Lemma 3.2.2 does not vanish.

Proof. Let $\psi_{0} \in E(T)$ be a non-zero eigenvector of $\left(c_{1}(F) \star_{0}\right)$ with eigenvalue $T$. We claim that $\psi_{0} \star_{0} H^{\prime}=0$. Take $\alpha \in H^{\prime}$ and write $\alpha=T \gamma-c_{1}(F) \star_{0} \gamma$. Then we have

$$
\psi_{0} \star \alpha=T \psi_{0} \star_{0} \gamma-\psi_{0} \star_{0} c_{1}(F) \star_{0} \gamma=0 .
$$

The claim follows. Write $1=c \psi_{0}+\psi^{\prime}$ with $\psi^{\prime} \in H^{\prime}$ and $c \in \mathbb{C}$. Applying $\left(\psi_{0} \star_{0}\right)$, we obtain $\psi_{0}=c \psi_{0} \star_{0} \psi_{0}$. Thus $c \neq 0$ and Lemma follows.

Remark 3.6.11. See $[15, \S 7]$ for a discussion of the limit formula in the classification problem of Fano manifolds.

3.7. Apery limit. We can replace the continuous limit of the ratio of the $J$-function appearing in Theorem 3.6.8 with the (discrete) limit of the ratio of the Taylor coefficients. Golyshev [31] considered such limits and called them Apery constants of Fano manifolds. A generalization to Apery class was studied by Galkin [23]. This limit sees the primitive part of the Gamma class.

Expand the $J$-function as

$$
J(t)=e^{c_{1}(F) \log t} \sum_{n \geqslant 0} J_{n} t^{n} .
$$


The coefficient $J_{n} \in H^{\bullet}(F)$ is given by:

$$
J_{n}:=\sum_{i=1}^{N} \sum_{d \in \operatorname{Eff}(F): c_{1}(F) \cdot d=n}\left\langle\phi_{i} \psi^{n-2-\operatorname{dim} \phi_{i}}\right\rangle_{0,1, d}^{F} \phi^{i}
$$

where we set $\operatorname{dim} \phi_{i}=\operatorname{dim} F-\frac{1}{2} \operatorname{deg} \phi_{i}$. See Remark 3.6.6. The main theorem in this section is stated as follows:

Theorem 3.7.1. Suppose that $F$ satisfies Property $\mathcal{O}$ and suppose also that the principal asymptotic class $A_{F}$ satisfies $\left\langle[\mathrm{pt}], A_{F}\right\rangle \neq 0$. Let $r$ be the Fano index. For every $\alpha \in H .(F)$ such that $c_{1}(F) \cap \alpha=0$, we have

$$
\liminf _{n \rightarrow \infty}\left|\frac{\left\langle\alpha, J_{r n}\right\rangle}{\left\langle[\mathrm{pt}], J_{r n}\right\rangle}-\frac{\left\langle\alpha, A_{F}\right\rangle}{\left\langle[\mathrm{pt}], A_{F}\right\rangle}\right|=0
$$

Remark 3.7.2. Note that $J_{n}=0$ unless $r$ divides $n$. In the left-hand side of the above formula, we set $\left\langle\alpha, J_{r n}\right\rangle /\left\langle[\mathrm{pt}], J_{r n}\right\rangle=\infty$ if $\left\langle[\mathrm{pt}], J_{r n}\right\rangle=0$. It is however expected that the following properties will hold for Fano manifolds:

- the Gromov-Witten invariants $\left\langle[\mathrm{pt}], J_{r n}\right\rangle$ are all positive for $n \geqslant 0$;

- $\lim \inf _{n \rightarrow \infty}$ in Theorem 3.7.1 can be replaced with $\lim _{n \rightarrow \infty}$.

Remark 3.7.3. More generally, if $\alpha, \beta \in H_{\bullet}(F)$ are homology classes such that $\alpha \cap c_{1}(F)=$ $\beta \cap c_{1}(F)=0$ and if we have $\left\langle\beta, A_{F}\right\rangle \neq 0$, we have the limit formula

$$
\liminf _{n \rightarrow \infty}\left|\frac{\left\langle\alpha, J_{r n}\right\rangle}{\left\langle\beta, J_{r n}\right\rangle}-\frac{\left\langle\alpha, A_{F}\right\rangle}{\left\langle\beta, A_{F}\right\rangle}\right|=0 .
$$

This can be shown by the same argument as below. We shall restrict to the case $\beta=[\mathrm{pt}]$ as Gamma Conjecture I implies $\left\langle[\mathrm{pt}], A_{F}\right\rangle \neq 0$.

Define the functions $G$ and $\widehat{G}$ as:

$$
\begin{aligned}
G(t) & :=\langle[\mathrm{pt}], J(t)\rangle=\sum_{n=0}^{\infty} G_{n} t^{n}, \quad \text { where } G_{n}:=\left\langle[\mathrm{pt}], J_{n}\right\rangle, \\
\widehat{G}(\kappa) & :=\sum_{n=0}^{\infty} n ! G_{n} \kappa^{n}=\frac{1}{\kappa} \int_{0}^{\infty} G(t) e^{-t / \kappa} d t .
\end{aligned}
$$

These functions $G, \widehat{G}$ are called (unregularized or regularized) quantum periods of Fano manifolds [15].

Lemma 3.7.4. Let $F$ be an arbitrary Fano manifold. The convergence radius of $\widehat{G}(\kappa)$ is bigger than or equal to $1 / T$.

Proof. Recall that we have $G(t)=(2 \pi t)^{-\operatorname{dim} F / 2}\left\langle\Phi^{\vee}([\mathrm{pt}])(z), 1\right\rangle$ (see (3.6.4)) and $\Phi^{\vee}([\mathrm{pt}])(z)$ is a $\nabla^{\vee}$-flat section (where $z=t^{-1}$ ). By Proposition 3.5.2, we have an estimate $\left\|\Phi([\mathrm{pt}])\left(t^{-1}\right)\right\| \leqslant$ $C_{0} e^{T t}$ on $t \in[1, \infty)$ for some constant $C_{0}>0$, and thus the following Laplace transformation converges for $\lambda$ with $\operatorname{Re}(\lambda)<-T$ and $\nu \gg 0, \nu \notin \mathbb{Z}$.

$$
\varphi(\lambda)=(2 \pi)^{-\frac{\operatorname{dim} F}{2}} \int_{0}^{\infty} t^{\nu-1} e^{\lambda t} \Phi^{\vee}([\mathrm{pt}])\left(t^{-1}\right) d t
$$

The condition $\nu \gg 0$ ensures that the integral converges near $t=0$ (since $\nabla^{\vee}$ is regular singular at $t=0, f\left(t^{-1}\right)$ is of at most polynomial growth near $\left.t=0\right)$. By the standard 
argument using integration by parts, we can show that $\varphi(\lambda)$ is flat for the connection $\widetilde{\nabla}^{(\nu)}$ given by

$$
\widetilde{\nabla}_{\partial_{\lambda}}^{(\nu)}=\partial_{\lambda}+\left(\lambda+\left(c_{1}(F) \star_{0}\right)^{\mathrm{t}}\right)^{-1}\left(-\mu^{\mathrm{t}}+\nu\right)
$$

Then the convergence radius of

$$
\begin{aligned}
\left\langle\varphi\left(-\kappa^{-1}\right), 1\right\rangle & =\int_{0}^{\infty} t^{\nu+\frac{\operatorname{dim} F}{2}-1} G(t) e^{-t / \kappa} d t \\
& =\kappa^{\nu+\frac{\operatorname{dim} F}{2}-1} \sum_{n=0}^{\infty} \Gamma\left(n+\nu+\frac{\operatorname{dim} F}{2}\right) G_{n} \kappa^{n}
\end{aligned}
$$

is bigger than or equal to $1 / T$ as $\widetilde{\nabla}^{(\nu)}$ has no singularities in $\{|\lambda|>T\}$. The function $\widehat{G}(\kappa)$ has the same radius of convergence as $\left\langle\varphi\left(-\kappa^{-1}\right), 1\right\rangle$ and the conclusion follows.

Lemma 3.7.6. Suppose that $F$ satisfies Property $\mathcal{O}$ and $\left\langle[\mathrm{pt}], A_{F}\right\rangle \neq 0$. Then the convergence radius of $\widehat{G}(\kappa)$ is exactly $1 / T$.

Proof. In view of the discussion in the previous lemma, it suffices to show that the function $\left\langle\varphi\left(-\kappa^{-1}\right), 1\right\rangle$ in (3.7.5) has a singularity at $\kappa=1 / T$. By (3.6.4) we have $G(t)=$ $(2 \pi t)^{-\operatorname{dim} F / 2}\left\langle\Phi^{\vee}([\mathrm{pt}])(z), 1\right\rangle$. Thus by Proposition 3.6.2 and Lemma 3.6.10, we have

$$
\lim _{t \rightarrow+\infty} e^{-t T}(2 \pi t)^{\frac{\operatorname{dim} F}{2}} G(t)=\left\langle[\mathrm{pt}], A_{F}\right\rangle\left\langle\psi_{0}^{*}, 1\right\rangle \neq 0 .
$$

Therefore the Laplace transform (3.7.5) diverges as $\kappa$ approaches $1 / T$ from the left for $\nu \gg$ 0 .

Proof of Theorem 3.7.1. Set $\beta:=\alpha-\frac{\left\langle\alpha, A_{F}\right\rangle}{\left\langle[\mathrm{pt}], A_{F}\right\rangle}[\mathrm{pt}]$. Then $\left\langle\beta, A_{F}\right\rangle=0$. It suffices to show that

$$
\liminf _{n \rightarrow \infty}\left|\frac{\left\langle\beta, J_{r n}\right\rangle}{\left\langle[\mathrm{pt}], J_{r n}\right\rangle}\right|=0 \text {. }
$$

Set $b_{n}:=\left\langle\beta, J_{n}\right\rangle$. Then we have $\sum_{n=0}^{\infty} b_{n} t^{n}=\langle\beta, J(t)\rangle=(2 \pi t)^{-\operatorname{dim} F / 2}\left\langle\Phi^{\vee}(\beta)\left(t^{-1}\right), 1\right\rangle$ by (3.6.4). By Proposition 3.6.2, we have $\operatorname{Lead}\left(\Phi^{\vee}(\beta)\right)=\left\langle\beta, A_{F}\right\rangle \psi_{0}^{*}=0$. Thus by Proposition 3.5.2 we have $\lim _{t \rightarrow+\infty} e^{-t \lambda} \Phi^{\vee}(\beta)\left(t^{-1}\right)=0$ for every $\lambda>T^{\prime}$. Consider again the Laplace transform

$$
\varphi_{\beta}(\lambda)=(2 \pi)^{-\frac{\operatorname{dim} F}{2}} \int_{0}^{\infty} t^{\nu-1} e^{t \lambda} \Phi^{\vee}(\beta)\left(t^{-1}\right) d t
$$

for $\nu \gg 0$ (as in the proof of Lemma 3.7.4). This is a $\widetilde{\nabla}^{(\nu)}$-flat section which is regular on $\left\{\lambda: \operatorname{Re}(\lambda)<-T^{\prime}\right\}$. Then its component

$$
\left\langle\varphi_{\beta}\left(-\kappa^{-1}\right), 1\right\rangle=\kappa^{\nu+\frac{\operatorname{dim} F}{2}-1} \sum_{n=0}^{\infty} \Gamma\left(n+\nu+\frac{\operatorname{dim} F}{2}\right) b_{n} \kappa^{n}
$$

has the radius of convergence strictly bigger than $1 / T$. This is because the only singularities of $\widetilde{\nabla}^{(\nu)}$ on $\{|\lambda| \geqslant T\}$ are $\left\{-e^{2 \pi \mathrm{i} k / r} T: k=0, \ldots, r-1\right\}$ and $\left\langle\varphi_{\beta}(\lambda), 1\right\rangle$ is regular at $\lambda=-T$ and satisfies $\left\langle\varphi_{\beta}\left(e^{2 \pi \mathrm{i} / r} \lambda\right), 1\right\rangle=e^{-\left(\nu+\frac{\operatorname{dim} F}{2}-1\right) 2 \pi \mathrm{i} / r}\left\langle\varphi_{\beta}(\lambda), 1\right\rangle$. (Here we used Part (2) of Property O.) This implies that there exist numbers $C, R>0$ such that $R>1 / T$ and

$$
\left|b_{n} n !\right| \leqslant C R^{-n} \text {. }
$$

On the other hand, Lemma 3.7.6 implies that

$$
\limsup _{n \rightarrow \infty}\left|G_{n} n !\right|^{1 / n}=T .
$$


Let $\epsilon>0$ be such that $(1-\epsilon) R T>1$. Then we have a number $n_{0}$ such that for every $n \geqslant n_{0}$ we have $\sup _{m \geqslant n}\left|G_{m} m !\right|^{1 / m} \geqslant T(1-\epsilon / 2)$. Namely we can find a sequence $n_{0} \leqslant n_{1}<n_{2}<$ $n_{3}<\cdots$ such that $\left|G_{n_{i}}\right| n_{i} ! \geqslant T^{n_{i}}(1-\epsilon)^{n_{i}}$. Therefore

$$
\left|\frac{b_{n_{i}}}{G_{n_{i}}}\right|=\left|\frac{b_{n_{i}} n_{i} !}{G_{n_{i}} n_{i} !}\right| \leqslant \frac{C R^{-n_{i}}}{T^{n_{i}}(1-\epsilon)^{n_{i}}}=\frac{C}{(R T(1-\epsilon))^{n_{i}}} .
$$

This implies $\liminf \operatorname{in}_{n \rightarrow \infty}\left|b_{n} / G_{n}\right|=0$, which proves (3.7.7).

3.8. Quantum cohomology central charges. For a vector bundle $V$ on $F$, we define

$$
Z(V):=(2 \pi z)^{\frac{\operatorname{dim} F}{2}}\left(1, \Phi\left(\widehat{\Gamma}_{F} \operatorname{Ch}(V)\right)(z)\right)_{F} .
$$

This quantity is called the quantum cohomology central charge ${ }^{7}$ of $V$ in [45]. Using the property $S(z)^{*}=S(-z)^{-1},(3.4 .1)$ and Definition 3.6.3, we can write $Z(V)$ in terms of the $J$-function:

$$
Z(V)=(2 \pi \mathrm{i})^{\operatorname{dim} F}\left[J\left(e^{\pi \mathrm{i}} t\right), \widehat{\Gamma}_{F} \mathrm{Ch}(V)\right)
$$

where $t=z^{-1}$ and $[\cdot, \cdot)$ is the pairing in (3.4.5). Therefore, as a component of the $J$-function, $Z(V)$ gives a scalar-valued solution to the quantum differential equations (see Remark 3.6.5). If $F$ satisfies Gamma Conjecture I, the central charge $Z(\mathcal{O})$ satisfies the following smallest asymptotics:

$$
Z(\mathcal{O}) \sim \sqrt{\left(\psi_{0}, \psi_{0}\right)_{F}}(2 \pi z)^{\frac{\operatorname{dim} F}{2}} e^{-T / z} \quad \text { as } z \rightarrow+0
$$

where $\psi_{0} \in E(T)$ is the idempotent for $\star_{0}$. This follows from Proposition 3.4.8 and the proof of Lemma 3.6.10.

\section{Gamma Conjecture II}

In this section we restrict to a Fano manifold $F$ with semisimple quantum cohomology and state a refinement of Gamma Conjecture I for such $F$. We represent the irregular monodromy of quantum connection by a marked reflection system given by a basis of asymptotically exponential flat sections. Gamma Conjecture II says that the marked reflection system coincides with a certain Gamma-basis $\left\{\widehat{\Gamma}_{F} \mathrm{Ch}\left(E_{i}\right)\right\}$ for some exceptional collection $\left\{E_{i}\right\}$ of the derived category $\mathcal{D}_{\text {coh }}^{b}(F)$. This also refines Dubrovin's conjecture [18].

4.1. Semiorthonormal basis and Gram matrix. Let $\mathcal{V}=\left(V ;[\cdot, \cdot) ; v_{1}, \ldots, v_{N}\right)$ be a triple of a vector space $V$, a bilinear form $[\cdot, \cdot)=[\cdot, \cdot)_{V}$, and a collection of vectors $v_{1}, \ldots, v_{N} \in V$. The bilinear form $[\cdot, \cdot)$ is not necessarily symmetric nor skew-symmetric. We say that $\mathcal{V}$ is a semiorthonormal collection if the Gram matrix $G_{i j}=\left[v_{i}, v_{j}\right)$ is uni-uppertriangular: $\left[v_{i}, v_{i}\right)=1$ for all $i$, and $\left[v_{i}, v_{j}\right)=0$ for all $i>j$. This implies that $G$ is non-degenerate, hence vectors $v_{i}$ are linearly independent so $N \leqslant \operatorname{dim} V$. We say that $\mathcal{V}$ is a semiorthonormal basis (SOB) if $N=\operatorname{dim} V$ i.e. $v_{i}$ form a basis of vector space $V$, this implies that pairing $[\cdot, \cdot)$ is non-degenerate.

Set of SOBs admits an obvious action of $(\mathbb{Z} / 2 \mathbb{Z})^{N}$ : if $v_{1}, \ldots, v_{N}$ is SOB then so is $\pm v_{1}, \ldots, \pm v_{N}$, in this case we say that SOBs are the same up to sign. For $u, v \in V$, define the right mutation $R_{u} v$ and the left mutation $L_{u} v$ by

$$
R_{u} v:=v-[v, u) u, \quad L_{u} v:=v-[u, v) u .
$$

\footnotetext{
${ }^{7}$ The central charge $Z(V)$ here is denoted by $(2 \pi i)^{\operatorname{dim} F} Z(V)$ in [45].
} 
The braid group $B_{N}$ on $N$ strands acts on the set of SOBs of $N$ vectors by:

$$
\begin{aligned}
\sigma_{i}\left(v_{1}, \ldots, v_{N}\right) & =\left(v_{1}, \ldots, v_{i-1}, v_{i+1}, R_{v_{i+1}} v_{i}, v_{i+2}, \ldots, v_{N}\right) \\
\sigma_{i}^{-1}\left(v_{1}, \ldots, v_{N}\right) & =\left(v_{1}, \ldots, v_{i-1}, L_{v_{i}} v_{i+1}, v_{i}, v_{i+2}, \ldots, v_{N}\right)
\end{aligned}
$$

where $\sigma_{1}, \ldots, \sigma_{N-1}$ are generators of $B_{N}$ and satisfy the braid relation

$$
\begin{aligned}
& \sigma_{i} \sigma_{i+1} \sigma_{i}=\sigma_{i+1} \sigma_{i} \sigma_{i+1} \\
& \sigma_{i} \sigma_{j}=\sigma_{j} \sigma_{i} \quad \text { if }|i-j| \geqslant 2 .
\end{aligned}
$$

4.2. Marked reflection system. A marked reflection system (MRS) of phase $\phi \in \mathbb{R}$ is a tuple $\left(V,[\cdot, \cdot),\left\{v_{1}, \ldots, v_{N}\right\}, m, e^{i \phi}\right)$ consisting of

- a complex vector space $V$;

- a bilinear form $[\cdot, \cdot)$ on $V$;

- an unordered basis $\left\{v_{1}, \ldots, v_{N}\right\}$ of $V$;

- a marking $m:\left\{v_{1}, \ldots, v_{N}\right\} \rightarrow \mathbb{C}$; we write $u_{i}=m\left(v_{i}\right)$ and $\mathbf{u}=\left\{u_{1}, \ldots, u_{N}\right\}$;

- $e^{\mathrm{i} \phi} \in S^{1} ; \phi \in \mathbb{R}$ is called a phase

such that the vectors $v_{i}$ are semiorthonormal in the sense that:

$$
\left[v_{i}, v_{j}\right)= \begin{cases}1 & \text { if } i=j \\ 0 & \text { if } i \neq j \text { and } \operatorname{Im}\left(e^{-i \phi} u_{i}\right) \leqslant \operatorname{Im}\left(e^{-\mathbf{i} \phi} u_{j}\right) .\end{cases}
$$

When the basis is ordered appropriately, $\left(V,[\cdot, \cdot), v_{1}, \ldots, v_{N}\right)$ gives an SOB. We say that $\phi \in \mathbb{R}$ (or $e^{\mathbf{i} \phi} \in S^{1}$ ) is admissible for a multiset $\mathbf{u}=\left\{u_{1}, \ldots, u_{N}\right\}$ in $\mathbb{C}$ if $e^{\mathbf{i} \phi}$ is not parallel to any non-zero difference $u_{i}-u_{j}$. An MRS is said to be admissible if the phase $\phi$ is admissible for u. By a small perturbation of the marking, one can always make an MRS admissible. The circle $S^{1}$ acts on the space of all MRSs by rotating simultaneously the phase and all markings: $\phi \mapsto \alpha+\phi, u_{i} \mapsto e^{\mathrm{i} \alpha} u_{i}$.

We write $h_{\phi}: \mathbb{C} \rightarrow \mathbb{R}$ for the $\mathbb{R}$-linear function $h_{\phi}(z)=\operatorname{Im}\left(e^{-i \phi} z\right)$.

Remark 4.2 .2 ([30]). The data of admissible marked reflection system may be used to produce a polarized local system on $\mathbb{C} \backslash \mathbf{u}$ (cf. the second structure connection [17, 54]): by identifying the fiber with $V$, endowing it with a bilinear form $\{\cdot, \cdot\}$ (either with the symmetric form $\left\{v_{1}, v_{2}\right\}=\left[v_{1}, v_{2}\right)+\left[v_{2}, v_{1}\right)$ or with the skew-symmetric form $\left.\left\{v_{1}, v_{2}\right\}=\left[v_{1}, v_{2}\right)-\left[v_{2}, v_{1}\right)\right)$, choosing " $e^{i \phi} \infty$ " for the base point, joining it with the points $u_{i}$ with the level rays $u_{i}+\mathbb{R}_{\geqslant 0} e^{i \phi}$ as paths, trivializing the local system outside the union of the paths, and requiring that the turn around $u_{i}$ act in the monodromy representation by the reflection with respect to $v_{i}$ : $r_{v_{i}}(v)=v-\left\{v, v_{i}\right\} v_{i}$. In case $u_{i}$ is a multiple marking all the respective reflections commute, so the monodromy of the local system is well-defined as a product of such reflections. The form $\{\cdot, \cdot\}$ could be degenerate, however construction above also provides local systems with fiber the quotient-space $V / \operatorname{Ker}\{\cdot, \cdot\}$ (use reflections with respect to images of $v_{i}$ in the quotientspaces).

When $u_{1}, \ldots, u_{N}$ are pairwise distinct, this construction could be reversed with a little ambiguity: if there is such a local system and the paths are given, we take $V$ to be a fiber, define $v_{i} \in V$ as a reflection vector of the monodromy at $u_{i}$ (which could be determined up to sign if $\{\cdot, \cdot\}$ is non-degenerate), and define $\left[v_{i}, v_{i}\right)=1$ for all $i$, for $i \neq j$ put $\left[v_{i}, v_{j}\right)=0$ if $h_{\phi}\left(u_{i}\right)<h_{\phi}\left(u_{j}\right)$ and $\left[v_{i}, v_{j}\right)=\left\{v_{i}, v_{j}\right\}$ otherwise. 
4.3. Mutation of MRSs. In this section, we explain how an MRS changes by mutation as we vary the marking $\mathbf{u}$ and the phase $\phi$. First we give an intuitive explanation and then give a formal definition.

The mutation of MRSs is parallel to the mutation of asymptotically exponential flat sections in $\S 2.6$ (see Corollary 2.6.6). For a given admissible $\operatorname{MRS}\left(V,[\cdot, \cdot),\left\{v_{1}, \ldots, v_{N}\right\}, m\left(v_{i}\right)=\right.$ $\left.u_{i}, e^{\mathrm{i} \phi}\right)$, we draw the ray $L_{i}=u_{i}+\mathbb{R}_{\geqslant 0} e^{\mathrm{i} \phi}$ from each marking $u_{i}$ in the direction of $\phi$ to get a picture as in Figure 6. We consider a variation of the parameters $\mathbf{u}$ and $\phi$. We allow that a multiple marking separates into distinct ones, but do not allow that two different markings $u_{i} \neq u_{j}$ collide unless the corresponding vectors $v_{i}, v_{j}$ are orthogonal $\left[v_{i}, v_{j}\right)=\left[v_{j}, v_{i}\right)=0$. The vectors $v_{1}, \ldots, v_{N}$ stay constant while each $u_{j}$ does not hit any other rays $L_{i}$ with $u_{j} \neq u_{i}$. When $u_{j}$ crosses a ray $L_{i}$ from the right side of $L_{i}$ (resp. the left side of $L_{i}$ ) towards the direction $\phi$, the vectors marked by $u_{i}$ undergo the right (resp. left) mutation. In the situation of Figure 7, if a vector $v_{l}$ is marked by $u_{i}$, it is transformed to

$$
v_{l}^{\prime}=\left(\prod_{k: u_{j}=u_{k}} R_{v_{k}}\right) v_{l}=v_{l}-\sum_{k: u_{j}=u_{k}}\left[v_{l}, v_{k}\right) v_{k}
$$

after the move. The vectors that are not assigned the marking $u_{i}$ remain the same. Note that the vectors with the same marking $u_{j}$ are orthogonal to each other and hence the above product of $R_{v_{k}}$ does not depend on the order. Note also that the vectors $v_{l}^{\prime}$ marked by $u_{i}$ are mutually orthogonal even after the mutation.

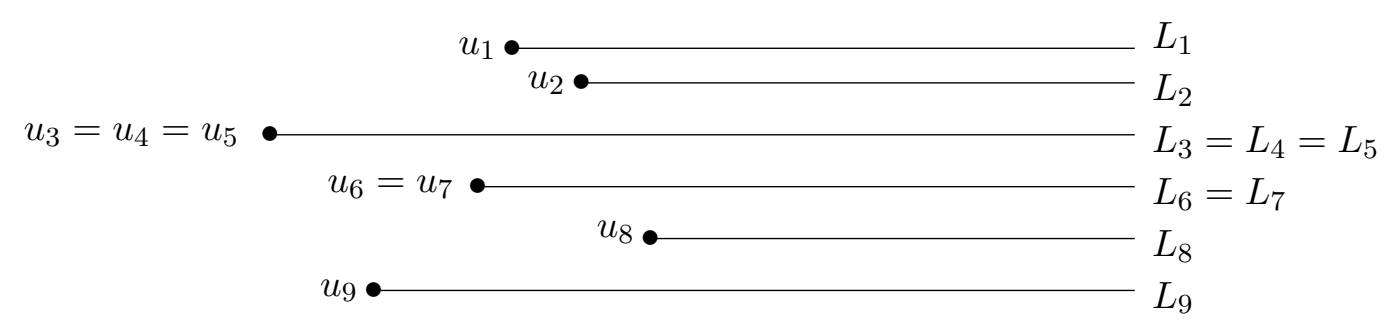

FiguRE 6 . Rays $L_{i}$ in the admissible direction $e^{\mathbf{i} \phi}=1$.

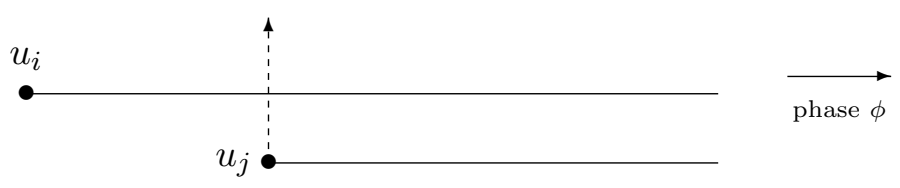

FIGURE 7. Right mutation

Remark 4.3.1. Let $\left(V,[\cdot, \cdot),\left\{v_{1}, \ldots, v_{N}\right\}, m\left(v_{i}\right)=u_{i}, e^{i \phi}\right)$ be an admissible MRS with multiple markings. Since we have $\left[v_{i}, v_{j}\right)=\left[v_{j}, v_{i}\right)=0$ if $u_{i}=u_{j}$ and $i \neq j$ (see (4.2.1)), there exists $\epsilon>0$ such that all the admissible MRSs of the form $\left(V,[\cdot, \cdot),\left\{v_{1}, \ldots, v_{N}\right\}, m\left(v_{i}\right)=u_{i}^{\prime}, e^{i \phi}\right)$ with pairwise distinct $u_{1}^{\prime}, \ldots, u_{N}^{\prime}$ and $\left|u_{i}-u_{i}^{\prime}\right|<\epsilon$ are related to each other by mutation. 
Now we give a formal and precise definition for the deformation of MRSs. We first deal with the case where $\mathbf{u}=\left(u_{1}, \ldots, u_{N}\right)$ are pairwise distinct, i.e. give an element of the configuration space $C_{N}(\mathbb{C})(2.7 .1)$. We construct a local system $\mathcal{L}=\mathcal{L}(V,[\cdot, \cdot))$ of MRSs with fixed $(V,[\cdot, \cdot))$ over $C_{N}(\mathbb{C}) \times S^{1}$. Then we extend the étalé space (sheaf space) of $\mathcal{L}$ to a branched cover $\mathfrak{M}$ over $S^{N}(\mathbb{C}) \times S^{1}$ (where $S^{N}(\mathbb{C})$ is the symmetric power of $\mathbb{C}$ ); this amounts to pushing forward $\mathcal{L}$ to $S^{N}(\mathbb{C}) \times S^{1}$ as an orbi-sheaf. We will see that the fiber $\mathfrak{M}_{\left(\mathbf{u}, e^{\mathrm{i} \phi}\right)}$ at $\left(\mathbf{u}, e^{\mathrm{i} \phi}\right) \in S^{N}(\mathbb{C}) \times S^{1}$ contains the set of all MRSs on $(V,[\cdot, \cdot))$ with marking $\mathbf{u}$ and phase $\phi$. Then we define a deformation of MRSs as a continuous path in $\mathfrak{M}$.

Local system on the 'distinct' locus. Consider the codimension-one stratum $W \subset$ $C_{N}(\mathbb{C}) \times S^{1}$ consisting of non-admissible parameters $\left(\mathbf{u}, e^{\mathbf{i} \phi}\right)$ :

$$
W=\left\{\left(\mathbf{u}, e^{\mathrm{i} \phi}\right) \in C_{N}(\mathbb{C}) \times S^{1}: h_{\phi}\left(u_{i}\right)=h_{\phi}\left(u_{j}\right) \text { for some } i \neq j\right\} .
$$

Note that the $S^{1}$-action $\left(\mathbf{u}, e^{\mathbf{i} \phi}\right) \mapsto\left(e^{\mathbf{i} \alpha} \mathbf{u}, e^{\mathbf{i}(\alpha+\phi)}\right)$ on $C_{N}(\mathbb{C}) \times S^{1}$ preserves $W$. The complement $\left(C_{N}(\mathbb{C}) \times S^{1}\right) \backslash W$ is a connected open subset. Fix a vector space $V$ with a bilinear form $[\cdot, \cdot)$. We define $\mathcal{L}^{\circ}=\mathcal{L}^{\circ}(V,[\cdot, \cdot))$ to be the trivial local system on the open subset $\left(C_{N}(\mathbb{C}) \times S^{1}\right) \backslash W$ whose fiber at $\left(\mathbf{u}, e^{i \phi}\right) \in\left(C_{N}(\mathbb{C}) \times S^{1}\right) \backslash W$ is given by

$$
\mathcal{L}_{\left(\mathbf{u}, e^{\mathrm{i} \phi}\right)}^{\circ}=\{\text { MRSs on }(V,[\cdot, \cdot)) \text { with marking } \mathbf{u} \text { and phase } \phi\}
$$

where by "MRS on $(V,[\cdot, \cdot))$ with marking $\mathbf{u}$ and phase $\phi$ " we mean an MRS of the form $\left(V,[\cdot, \cdot),\left\{v_{1}, \ldots, v_{N}\right\}, m, e^{\mathbf{i} \phi}\right)$ such that $\mathbf{u}=\left\{m\left(v_{1}\right), \ldots, m\left(v_{N}\right)\right\}$. Note that the fiber $\mathcal{L}_{\left(\mathbf{u}, e^{\mathrm{i} \phi}\right)}^{\circ}$ is canonically identified with the set of SOBs on $V$ via the ordering given by $h_{\phi}$, i.e. we can order $v_{1}, \ldots, v_{N}$ in such a way that $h_{\phi}\left(u_{1}\right)>h_{\phi}\left(u_{2}\right)>\cdots>h_{\phi}\left(u_{N}\right)$ with $u_{i}=m\left(v_{i}\right)$. This identification defines the structure of a trivial local system on $\mathcal{L}^{\circ}$. Next we extend $\mathcal{L}^{\circ}$ to a local system $\mathcal{L}=\mathcal{L}(V,[\cdot, \cdot))$ on $C_{N}(\mathbb{C}) \times S^{1}$. Let $\jmath:\left(C_{N}(\mathbb{C}) \times S^{1}\right) \backslash W \rightarrow C_{N}(\mathbb{C}) \times S^{1}$ denote the inclusion. An extension of $\mathcal{L}^{\circ}$ is given by an action of $\pi_{1}\left(C_{N}(\mathbb{C}) \times S^{1}\right)$ on the set of SOBs of $V$ which is trivial on $\jmath_{*} \pi_{1}\left(\left(C_{N}(\mathbb{C}) \times S^{1}\right) \backslash W\right)$. Via the $S^{1}$-action on $C_{N}(\mathbb{C}) \times S^{1}$, we have the isomorphism

$$
\left\{\left(u_{1}, \ldots, u_{N}\right) \in \mathbb{C}^{N}: \operatorname{Im}\left(u_{1}\right)>\operatorname{Im}\left(u_{2}\right)>\cdots>\operatorname{Im}\left(u_{N}\right)\right\} \times S^{1} \cong\left(C_{N}(\mathbb{C}) \times S^{1}\right) \backslash W
$$

which sends $\left(\left(u_{1}, \ldots, u_{N}\right), e^{\mathbf{i} \phi}\right)$ to $\left(\left\{e^{\mathbf{i} \phi} u_{1}, \ldots, e^{\mathbf{i} \phi} u_{N}\right\}, e^{\mathbf{i} \phi}\right)$. Since the first factor of the lefthand side is contractible, we have $\pi_{1}\left(\left(C_{N}(\mathbb{C}) \times S^{1}\right) \backslash W\right) \cong \mathbb{Z}$ and the image of $\jmath_{*}: \pi_{1}\left(\left(C_{N}(\mathbb{C}) \times\right.\right.$ $\left.\left.S^{1}\right) \backslash W\right) \rightarrow \pi_{1}\left(C_{N}(\mathbb{C}) \times S^{1}\right)$ is generated by the class of an $S^{1}$-orbit. Since $\pi_{1}\left(C_{N}(\mathbb{C}) \times S^{1}\right)$ is the direct product of $\pi_{1}\left(C_{N}(\mathbb{C}) \times\{1\}\right)$ and $\pi_{1}$ of an $S^{1}$-orbit, an extension of the local system $\mathcal{L}^{\circ}$ to $C_{N}(\mathbb{C}) \times S^{1}$ is given by the action of the braid group $B_{N} \cong \pi_{1}\left(C_{N}(\mathbb{C}) \times\{1\}\right)$ on the set of SOBs of $V$. We use the $B_{N}$-action on SOBs described in $\S 4.1$ to define $\mathcal{L}$. More precisely, choosing

$$
\left(\mathbf{u}^{\circ}=\left\{u_{1}=(N-1) \mathbf{i}, u_{2}=(N-2) \mathbf{i}, \ldots, u_{N}=0\right\}, e^{\mathbf{i} \phi}=1\right)
$$

as a base point of $\left(C_{N}(\mathbb{C}) \times S^{1}\right) \backslash W$, we define the parallel transport along the closed path in Figure 8 (based at $\left(\mathbf{u}^{\circ}, 1\right)$ ) as the action of $\sigma_{i} \in B_{N}$ on SOBs given in (4.1.1). This defines the local system $\mathcal{L}$ of sets over $C_{N}(\mathbb{C}) \times S^{1}$.

Extension to the 'non-distinct' locus. Consider the following diagram:

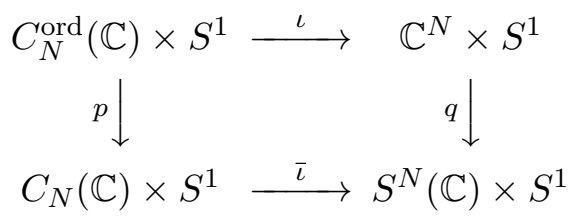




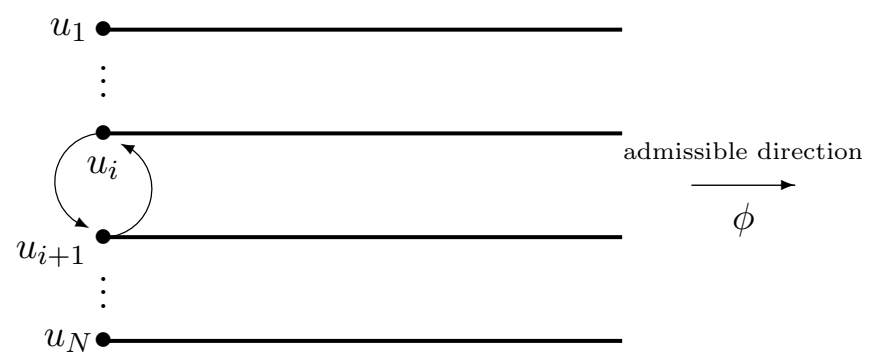

FiguRe 8. Braid $\sigma_{i}$

where $C_{N}^{\text {ord }}(\mathbb{C})=\left\{\left(u_{1}, \ldots, u_{N}\right) \in \mathbb{C}^{N}: u_{i} \neq u_{j}\right.$ for all $\left.i \neq j\right\}$ is the configuration space of ordered distinct $N$ points on $\mathbb{C}, S^{N}(\mathbb{C})=\mathbb{C}^{N} / \mathfrak{S}_{N}$ is the symmetric product of $\mathbb{C}, \iota, \bar{\iota}$ are the natural inclusions and $p, q$ are the natural projections. The local system $p^{*} \mathcal{L}$ on $C_{N}^{\text {ord }}(\mathbb{C}) \times S^{1}$ can be extended to a constructible sheaf $\iota_{*} p^{*} \mathcal{L}$ on $\mathbb{C}^{N} \times S^{1}$. Since $p$ is an $\mathfrak{S}_{N^{-}}$-covering, $p^{*} \mathcal{L}$ and $\iota_{*} p^{*} \mathcal{L}$ are naturally $\mathfrak{S}_{N}$-equivariant. We topologize the space of MRSs on $(V,[\cdot, \cdot))$ using the étalé space (also known as the "sheaf space") of the sheaf $\iota_{*} p^{*} \mathcal{L}$. We define:

$$
\mathfrak{M}:=\widetilde{\mathfrak{M}} / \mathfrak{S}_{N}, \quad \widetilde{\mathfrak{M}}:=\text { the étale space of the sheaf } \iota_{*} p^{*} \mathcal{L} \text {. }
$$

As we will see below, $\mathfrak{M}$ is a smooth manifold (which is non-separable if $N \geqslant 2$ ) and is a branched covering of $S^{N}(\mathbb{C}) \times S^{1}$. We will also see that $\mathfrak{M}$ contains the set of all MRSs on $(V,[\cdot, \cdot))$ as an open dense subset. We say that two MRSs on $(V,[\cdot, \cdot))$ are related by mutation (or mutation-equivalent) if they can be connected by a continuous path in $\mathfrak{M}$.

Remark 4.3.2. Using the language of orbifolds, we can regard $\mathfrak{M}$ as the étalé space of the orbi-sheaf $\bar{\iota}_{*} \mathcal{L}$ on $S^{N}(\mathbb{C})$.

Let $N_{\bullet}=\left(N_{1} \geqslant N_{2} \geqslant \cdots \geqslant N_{k}\right)$ be a partition of $N$ (i.e. $\left.N=\sum_{i=1}^{k} N_{i}\right)$. Define a locally closed subset $S\left(N_{\bullet}\right) \subset S^{N}(\mathbb{C})$ to be the set of multisets $\mathbf{u}$ of the form

$$
\mathbf{u}=(\overbrace{\lambda_{1}, \ldots, \lambda_{1}}^{N_{1}}, \overbrace{\lambda_{2}, \ldots, \lambda_{2}}^{N_{2}}, \cdots, \overbrace{\lambda_{k}, \ldots, \lambda_{k}}^{N_{k}})
$$

for some distinct $k$ points $\lambda_{1}, \ldots, \lambda_{k} \in \mathbb{C}$. We have

$$
S^{N}(\mathbb{C})=\bigsqcup_{N_{\bullet}: \text { partition of } N} S\left(N_{\bullet}\right)
$$

Define $\widetilde{S}\left(N_{\bullet}\right):=q^{-1}\left(S\left(N_{\bullet}\right)\right) \subset \mathbb{C}^{N}$. We have the following proposition.

Proposition 4.3.4. Let $N_{\bullet}=\left(N_{1} \geqslant N_{2} \geqslant \cdots \geqslant N_{k}\right)$ be a partition of $N$. The restriction of $\iota_{*} p^{*} \mathcal{L}$ to the stratum $\widetilde{S}\left(N_{\bullet}\right) \times S^{1}$ is a local system whose fiber is isomorphic to the set of "block semiorthonormal" bases of $(V,[\cdot, \cdot))$, i.e. bases $\left(v_{i, j}\right)_{1 \leqslant i \leqslant k, 1 \leqslant j \leqslant N_{i}}$ of $V$ such that

$$
\left[v_{i_{1}, j_{1}}, v_{i_{2}, j_{2}}\right)= \begin{cases}0 & \text { if } i_{1} \geqslant i_{2} \text { and }\left(i_{1}, j_{1}\right) \neq\left(i_{2}, j_{2}\right) \\ 1 & \text { if }\left(i_{1}, j_{1}\right)=\left(i_{2}, j_{2}\right) .\end{cases}
$$

The stabilizer $\operatorname{Stab}(\mathbf{u})=\mathfrak{S}_{N_{1}} \times \cdots \times \mathfrak{S}_{N_{k}}$ of the $\mathfrak{S}_{N}$-action on $\mathbb{C}^{N}$ at $\mathbf{u} \in \widetilde{S}\left(N_{\bullet}\right)$ acts on the stalk $\left(\iota^{*} p_{*} \mathcal{L}\right)_{\left(\mathbf{u}, e^{\mathrm{i} \phi}\right)}$ by permutation of the vectors $\left(v_{i, j}\right)$ in the same block. 
Proof. Let $\mathbf{u} \in \widetilde{S}\left(N_{\bullet}\right)$ be an element of the form (4.3.3). Let $D_{i} \subset \mathbb{C}, i=1, \ldots, k$ be pairwise disjoint open discs such that $\lambda_{i} \in D_{i}$. We consider a $\operatorname{Stab}(\mathbf{u})$-invariant open set $O$ containing $\left(\mathbf{u}, e^{\mathrm{i} \phi}\right)$ given by:

$$
O=D_{1}^{N_{1}} \times \cdots \times D_{k}^{N_{k}} \times I \subset \mathbb{C}^{N} \times S^{1}
$$

where $I \subset S^{1}$ is an interval containing $e^{\mathrm{i} \phi}$. The stalk $\left(\iota_{*} p^{*} \mathcal{L}\right)_{\left(\mathbf{u}, e^{i \phi}\right)}$ can be identified with the space of sections of $p^{*} \mathcal{L}$ over

$$
O \cap\left(C_{N}^{\text {ord }}(\mathbb{C}) \times S^{1}\right)=C_{N_{1}}^{\text {ord }}\left(D_{1}\right) \times \cdots \times C_{N_{k}}^{\text {ord }}\left(D_{k}\right) \times I
$$

where $C_{N_{i}}^{\text {ord }}\left(D_{i}\right)$ denotes the set of ordered distinct $N_{i}$ points on $D_{i}$. The fundamental group of $O \cap\left(C_{N}^{\text {ord }}(\mathbb{C}) \times S^{1}\right)$ is the product $P\left(N_{\bullet}\right):=P_{N_{1}} \times \cdots \times P_{N_{k}}$ of the pure braid groups $P_{N_{i}} \cong \pi_{1}\left(C_{N_{i}}^{\text {ord }}\left(D_{i}\right)\right)$. We choose a reference point

$$
\left(\mathbf{x}=\left(x_{i, j}\right)_{1 \leqslant i \leqslant k, 1 \leqslant j \leqslant N_{i}}, e^{\mathrm{i} \theta}\right) \in O \cap\left(C_{N}^{\mathrm{ord}}(\mathbb{C}) \times S^{1}\right)
$$

such that

- $\left(x_{i, 1}, \ldots, x_{i, N_{i}}\right) \in C_{N_{i}}^{\mathrm{ord}}\left(D_{i}\right)$ for $1 \leqslant i \leqslant k, e^{\mathrm{i} \theta} \in I$;

- $h_{\theta}\left(x_{i, 1}\right)>h_{\theta}\left(x_{i, 2}\right)>\cdots>h_{\theta}\left(x_{i, N_{i}}\right)$ for all $1 \leqslant i \leqslant k$;

- the intervals $\left[h_{\theta}\left(x_{i, 1}\right), h_{\theta}\left(x_{i, N_{i}}\right)\right], i=1, \ldots, k$ are mutually disjoint.

Renumbering the indices $i$, we may further assume that $h_{\theta}\left(x_{1,1}\right)>h_{\theta}\left(x_{2,1}\right)>\cdots>h_{\theta}\left(x_{k, 1}\right)$ (then $N_{1} \geqslant \cdots \geqslant N_{k}$ may no longer hold). With this choice, we can identify $\left(\iota_{*} p^{*} \mathcal{L}\right)_{\left(\mathbf{u}, e^{i \phi}\right)}$ with $\left(p^{*} \mathcal{L}_{\left(\mathbf{x}, e^{\mathrm{i} \theta}\right)}\right)^{P\left(N_{\bullet}\right)}$, i.e. the set of MRSs on $(V,[\cdot, \cdot))$ with marking $\mathbf{x}$ and phase $\theta$ which are $P\left(N_{\bullet}\right)$-invariant. The stalks $\left(\iota_{*} p^{*} \mathcal{L}\right)_{\left(\mathbf{u}^{\prime}, e^{\mathrm{i} \phi^{\prime}}\right)}$ at other points $\left(\mathbf{u}^{\prime}, e^{\mathrm{i} \phi^{\prime}}\right)$ in $O \cap\left(\widetilde{S}\left(N_{\bullet}\right) \times S^{1}\right)$ can be also identified with the same set, and therefore $\iota_{*} p^{*} \mathcal{L}$ is locally constant along the stratum $\widetilde{S}\left(N_{\bullet}\right) \times S^{1}$. On the other hand, it is easy to check that a semiorthonormal collection $\left(v_{1}, \ldots, v_{a}\right)$ is invariant under the pure braid group $P_{a} \subset B_{a}$ if and only if $v_{1}, \ldots, v_{a}$ are mutually orthogonal, i.e. $\left[v_{i}, v_{j}\right)=\left[v_{j}, v_{i}\right)=0$ for all $1 \leqslant i, j \leqslant a$. Therefore $P\left(N_{\bullet}\right)$-invariant MRSs with marking $\mathbf{x}$ and phase $\theta$ corresponds to block SOBs $\left(v_{i, j}\right)$ in the proposition.

To see the last statement, note that $\omega=\left(\omega_{1}, \ldots, \omega_{k}\right) \in \mathfrak{S}_{N_{1}} \times \cdots \times \mathfrak{S}_{N_{k}}$ sends a block SOB $\left(v_{i, j}\right)$ defining an element of $\left(p^{*} \mathcal{L}_{\left(\mathbf{x}, e^{\mathrm{i} \theta}\right)}\right)^{P\left(N_{\bullet}\right)}$ to a block SOB $\left(v_{i, \omega_{i}(j)}\right)$ defining an element of $\left(p^{*} \mathcal{L}_{\left(\omega \cdot \mathbf{x}, e^{\mathrm{i} \theta}\right)}\right)^{P\left(N_{\bullet}\right)}$ where $\omega \cdot \mathbf{x}=\left(x_{i, \omega_{i}(j)}\right)_{i, j}$. Since $\left(v_{i, 1}, \ldots, v_{i, N_{i}}\right)$ are mutually orthogonal, the parallel transport from $p^{*} \mathcal{L}_{\left(\omega \cdot \mathbf{x}, e^{\mathrm{i} \theta}\right)}$ to $p^{*} \mathcal{L}_{\left(\mathbf{x}, e^{\mathrm{i} \theta}\right)}$ sends the $\operatorname{SOB}\left(v_{i, \omega_{i}(j)}\right)$ to the $\operatorname{SOB}\left(v_{i, \omega_{i}(j)}\right)$, and the conclusion follows.

The above proposition shows that the $\mathfrak{S}_{N}$-action on the étale space $\widetilde{\mathfrak{M}}$ of $\iota_{*} p^{*} \mathcal{L}$ is free, and thus the quotient $\mathfrak{M}=\widetilde{\mathfrak{M}} / \mathfrak{S}_{N}$ is a smooth manifold. The natural map $\mathfrak{M} \rightarrow S^{N}(\mathbb{C}) \times S^{1}$ is a covering map restricted to each stratum $S\left(N_{\bullet}\right) \times S^{1}$. We show that MRSs on $(V,[\cdot, \cdot))$ form an open dense subset of $\mathfrak{M}$.

Proposition 4.3.8. For $\left(\mathbf{u}, e^{i \phi}\right) \in S^{N}(\mathbb{C}) \times S^{1}$, we have a canonical inclusion

$$
\{\text { MRSs on }(V,[\cdot, \cdot)) \text { with marking } \mathbf{u} \text { and phase } \phi\} \subset \mathfrak{M}_{\left(\mathbf{u}, e^{i \phi}\right)}
$$

where $\mathfrak{M}_{\left(\mathbf{u}, e^{i \phi}\right)}$ denotes the fiber of $\mathfrak{M} \rightarrow S^{N}(\mathbb{C}) \times S^{1}$ at $\left(\mathbf{u}, e^{\mathbf{i} \phi}\right)$. This inclusion is an equality if the phase $\phi$ is admissible for $\mathbf{u}$.

Proof. The fiber of $\mathfrak{M}$ at $\left(\mathbf{u}, e^{i \phi}\right) \in S^{N}(\mathbb{C}) \times S^{1}$ is given by $\left(\iota_{*} p^{*} \mathcal{L}\right)_{\left(\mathbf{u}, e^{i \phi}\right)} / \operatorname{Stab}(\mathbf{u})$. Here, by abuse of notation, $\mathbf{u}$ denotes both a point of $S^{N}(\mathbb{C})$ and its lift in $\mathbb{C}^{N}$. Suppose that $\mathbf{u} \in S\left(N_{\bullet}\right)$ is of the form (4.3.3). Let $\left(V,[\cdot, \cdot),\left\{v_{i, j}\right\}_{1 \leqslant i \leqslant k, 1 \leqslant j \leqslant N_{i}}\right)$ be an SOB such that the marking $m\left(v_{i, j}\right)=\lambda_{i}$ defines an MRS of phase $\phi$. We first construct an element of the 
fiber $\mathfrak{M}_{\left(\mathbf{u}, e^{i \phi}\right)}$ from these data. Take a sufficiently small open neighbourhood $O \subset \mathbb{C}^{N} \times S^{1}$ of $\left(\mathbf{u}, e^{\mathrm{i} \phi}\right)$ of the form (4.3.6) such that, for any $\left(\mathbf{x}=\left(x_{i, j}\right)_{1 \leqslant i \leqslant k, 1 \leqslant j \leqslant N_{i}}, e^{\mathrm{i} \theta}\right) \in O \cap\left(C_{N}^{\text {ord }}(\mathbb{C}) \times S^{1}\right)$,

$$
h_{\phi}\left(\lambda_{i_{1}}\right)>h_{\phi}\left(\lambda_{i_{2}}\right) \Longrightarrow \forall j_{1}, \forall j_{2}, h_{\theta}\left(x_{i_{1}, j_{1}}\right)>h_{\theta}\left(x_{i_{2}, j_{2}}\right) .
$$

For $\left(\mathbf{x}, e^{\mathrm{i} \theta}\right) \in O \cap\left(C_{N}^{\text {ord }}(\mathbb{C}) \times S^{1} \backslash p^{-1}(W)\right)$, we define an MRS $\mathfrak{R e f}(\mathbf{x}, \theta)$ by

$$
\mathfrak{R e f}(\mathbf{x}, \theta):=\left(V,[\cdot, \cdot),\left\{v_{i, j}\right\}, m\left(v_{i, j}\right)=x_{i, j}, e^{\mathrm{i} \theta}\right) .
$$

This is indeed an MRS because $v_{i, j}$ 's with the same value of $h_{\phi}\left(\lambda_{i}\right)$ are orthogonal to each other. For the same reason, the family of MRSs $\mathfrak{R e f}(\mathbf{x}, \theta)$ parametrized by $\left(\mathbf{x}, e^{\mathbf{i} \theta}\right) \in O \cap$ $\left(C_{N}^{\text {ord }}(\mathbb{C}) \times S^{1} \backslash p^{-1}(W)\right)$ are related to each other by mutation, and extends to a locally constant section of $p^{*} \mathcal{L}$ over $O \cap\left(C_{N}^{\text {ord }}(\mathbb{C}) \times S^{1}\right)$. Therefore, they define an element of the stalk $\left(\iota_{*} p^{*} \mathcal{L}\right)_{\left(\mathbf{u}, e^{i \phi}\right)}$. This construction depends on an auxiliary choice of the ordering of each orthogonal collection $\left\{v_{i, 1}, \ldots, v_{i, N_{i}}\right\}$, and changing this choice yields elements in a single $\operatorname{Stab}(\mathbf{u})$-orbit in $\left(\iota_{*} p^{*} \mathcal{L}\right)_{\left(\mathbf{u}, e^{\mathrm{i} \phi}\right)}$. Thus we get an injection from the set of MRSs with marking $\mathbf{u}$ and phase $\phi$ to $\mathfrak{M}_{\left(\mathbf{u}, e^{i \phi}\right)} \cong\left(\iota_{*} p^{*} \mathcal{L}\right)_{\left(\mathbf{u}, e^{i \phi}\right)} / \operatorname{Stab}(\mathbf{u})$. When $\phi$ is admissible for $\mathbf{u}$, every point $\left(\mathbf{x}, e^{\mathrm{i} \theta}\right)$ in $O \cap\left(C_{N}^{\text {ord }}(\mathbb{C}) \times S^{1} \backslash p^{-1}(W)\right)$ satisfies the conditions for the reference point (4.3.7) in the proof of Proposition 4.3.4. Thus the argument and the result there show that this construction is an isomorphism.

4.4. Exceptional collections. In this section we sketch only the necessary definitions of (full) exceptional collections in triangulated categories and braid group action on them. For details we refer the reader to [7] or to the survey [34].

Recall that an object $E$ in a triangulated category $\mathcal{T}$ over $\mathbb{C}$ is called exceptional if $\operatorname{Hom}(E, E)=\mathbb{C}$ and $\operatorname{Hom}(E, E[j])=0$ for all $j \neq 0$. An ordered tuple of exceptional objects $E_{1}, \ldots, E_{N}$ is called an exceptional collection if $\operatorname{Hom}\left(E_{i}, E_{j}[k]\right)=0$ for all $i, j, k$ with $i>j$ and $k \in \mathbb{Z}$. If $E_{1}, \ldots, E_{N}$ is an exceptional collection and $k_{1}, \ldots, k_{N}$ is a set of integers then $E_{1}\left[k_{1}\right], \ldots, E_{N}\left[k_{N}\right]$ is also an exceptional collection, in this case we say that two collections coincide up to shift. An exceptional object $E$ gives a pair of functors called left mutation $L_{E}$ and right mutation $R_{E}$ which fit into the distinguished triangles ${ }^{8}$ :

$$
\begin{gathered}
L_{E} F[-1] \rightarrow \operatorname{Hom}^{\bullet}(E, F) \otimes E \rightarrow F \rightarrow L_{E} F, \\
R_{E} F \rightarrow F \rightarrow \operatorname{Hom}^{\bullet}(F, E)^{*} \otimes E \rightarrow R_{E} F[1] .
\end{gathered}
$$

The set of exceptional collections of $N$ objects in $\mathcal{T}$ admits an action of the braid group $B_{N}$ in a way parallel to the $B_{N}$-action (4.1.1) on SOBs, i.e. the generator $\sigma_{i}$ replaces $\left\{E_{i}, E_{i+1}\right\}$ with $\left\{E_{i+1}, R_{E_{i+1}} E_{i}\right\}$ and $\sigma_{i}^{-1}$ replaces $\left\{E_{i}, E_{i+1}\right\}$ with $\left\{L_{E_{i}} E_{i+1}, E_{i}\right\}$.

The subcategory in $\mathcal{T}$ generated by $E_{i}$ (minimal full triangulated subcategory that contains all $\left.E_{i}\right)$ stays invariant under mutations. An exceptional collection is called full if it generates the whole category $\mathcal{T}$; clearly braid group and shift action respects fullness. When a triangulated category $\mathcal{T}$ has a full exceptional collection $E_{1}, \ldots, E_{N}$, the Grothendieck $K$-group $K^{0}(\mathcal{T})$ is the abelian group freely generated by the classes $\left[E_{i}\right]$. The Gram matrix $G_{i j}=\chi\left(E_{i}, E_{j}\right)$ of the Euler pairing $\chi(E, F)=\sum(-1)^{i} \operatorname{dim} \operatorname{Hom}(E, F[i])$ is uniuppertriangular in the basis $\left\{\left[E_{i}\right]\right\}$. Thus any exceptional collection $E_{1}, \ldots, E_{N}$ produces an $\operatorname{SOB}\left(K^{0}(\mathcal{T}) \otimes \mathbb{C} ; \chi(\cdot, \cdot) ;\left[E_{1}\right], \ldots,\left[E_{n}\right]\right)$. The action of the braid group $B_{N}$ and shifts $E_{i} \rightarrow E_{i}\left[k_{i}\right]$ described above descends to the action of $B_{N}$ and change of signs on the $K$ group described in $\S 4.1$.

\footnotetext{
${ }^{8}$ What we write as $L_{E} F, R_{E} F$ here are denoted respectively by $L_{E} F[1], R_{E} F[-1]$ in $[7,34]$.
} 
4.5. Asymptotic basis and MRS of a Fano manifold. Let $F$ be a Fano manifold such that $\star_{\tau}$ is convergent and semisimple for some $\tau \in H^{*}(F)$. Choose a phase $\phi \in \mathbb{R}$ that is admissible for the spectrum of $\left(E \star_{\tau}\right)$. By Proposition 2.5.1, we have a basis $y_{1}(\tau, z), \ldots, y_{N}(\tau, z)$ of asymptotically exponential flat sections for the quantum connection $\left.\nabla\right|_{\tau}$ defined over the sector $\phi-\frac{\pi}{2}-\epsilon<\arg z<\phi+\frac{\pi}{2}+\epsilon$ for some $\epsilon>0$. They are canonically defined up to sign and ordering. The basis is marked by the spectrum $\mathbf{u}=\left\{u_{1}, \ldots, u_{N}\right\}$ of $\left(E_{\star_{\tau}}\right)$. We can naturally identify $y_{i}(\tau, z)$ on the universal cover of the $z$-plane $\mathbb{C}^{\times}$. By $\nabla$-parallel transportation of $y_{i}(\tau, z)$ to $\tau=0, \arg z=0$ and $|z| \gg 1$, we can identify the basis $y_{1}, \ldots, y_{N}$ of flat sections with a basis $A_{1}, \ldots, A_{N}$ of $H^{\bullet}(F)$ via the fundamental solution $S(z) z^{-\mu} z^{\rho}$ in Proposition 2.3.1, i.e.

$$
\left.y_{i}(\tau, z)\right|_{\substack{\text { parallel transport } \\ \text { to } \tau=0, \arg z=0}}=\Phi\left(A_{i}\right)(z):=(2 \pi)^{-\frac{\operatorname{dim} F}{2}} S(z) z^{-\mu} z^{\rho} A_{i}
$$

where $\Phi$ was given in (3.4.1). The basis $\left\{A_{1}, \ldots, A_{N}\right\}$ is defined up to sign and ordering and is semiorthonormal with respect to the pairing $[\cdot, \cdot)$ in $(3.4 .4)-(3.4 .5)$ by Proposition 2.6.4. We call it the asymptotic basis of $F$ at $\tau$ with respect to the phase $\phi$.

The marked reflection system (MRS) of $F$ is defined to be the tuple:

$$
\mathfrak{R e f}(F, \tau, \phi):=\left(H^{\bullet}(F),[\cdot, \cdot),\left\{A_{1}, \ldots, A_{N}\right\}, m: A_{i} \mapsto u_{i}, e^{\mathbf{i} \phi}\right) .
$$

The marking $m$ assigns to $A_{i}$ the corresponding eigenvalue $u_{i}$ of $\left(E \star_{\tau}\right)$. It is defined up to sign. Note that $\mathfrak{R e f}(F, \tau, \phi)$ and $\mathfrak{R e f}(F, \tau, \phi+2 \pi)$ are not necessarily the same: they differ by the monodromy around $z=0$. By the discussion in $\S 2.6$, when $(\tau, \phi)$ varies, the corresponding MRSs change by mutation as described in the previous section $\S 4.3$.

Remark 4.5.1. When $\star_{0}$ is semisimple, we have the two $\operatorname{MRSs} \mathfrak{R e f}(F, 0, \pm \epsilon)$ defined at the canonical position $\tau=0$ for a sufficiently small $\epsilon>0$. When $\phi=0$ is admissible for the spectrum of $\left(c_{1}(F) \star_{0}\right)$, the two MRSs coincide.

4.6. Dubrovin's (original) conjecture and Gamma conjecture II. We recall Dubrovin's Conjecture (see also Remark 4.6.5). Let $F$ be a Fano manifold. Dubrovin [18, Conjecture 4.2.2] conjectured:

(1) the quantum cohomology of $F$ is semisimple if and only if $\mathcal{D}_{\text {coh }}^{b}(F)$ admits a full exceptional collection $E_{1}, \ldots, E_{N}$;

and if the quantum cohomology of $F$ is semisimple, there exists a full exceptional collection $E_{1}, \ldots, E_{N}$ of $\mathcal{D}_{\text {coh }}^{b}(F)$ such that:

(2) the Stokes matrix $S=\left(S_{i j}\right)$ is given by $S_{i j}=\chi\left(E_{i}, E_{j}\right), i, j=1, \ldots, N$;

(3) the central connection matrix has the form $C=C^{\prime} C^{\prime \prime}$ when the columns of $C^{\prime \prime}$ are the components of $\mathrm{Ch}\left(E_{j}\right) \in H^{\bullet}(F)$ and $C^{\prime}: H^{\bullet}(F) \rightarrow H^{\bullet}(F)$ is some operator satisfying $C^{\prime}\left(c_{1}(F) a\right)=c_{1}(F) C^{\prime}(a)$ for any $a \in H^{\bullet}(F)$.

The central connection matrix $C$ above is given by

$$
C=\frac{1}{(2 \pi)^{\operatorname{dim} F / 2}}\left(\begin{array}{ccc}
\mid & & \mid \\
A_{1} & \cdots & A_{N} \\
\mid & & \mid
\end{array}\right)
$$

where the column vectors $A_{1}, \ldots, A_{N} \in H^{\bullet}(F)$ are an asymptotic basis in $\S 4.5$. Gamma Conjecture II makes precise the operator $C^{\prime}$ in Part (3) of Dubrovin's conjecture.

Conjecture 4.6.1 (Gamma Conjecture II). Let $F$ be a Fano manifold such that the quantum product $\star_{\tau}$ is convergent and semisimple for some $\tau \in H^{\bullet}(F)$ and that $\mathcal{D}_{\text {coh }}^{b}(F)$ has a full exceptional collection. There exists a full exceptional collection $\left\{E_{1}, \ldots, E_{N}\right\}$ such that the 
asymptotic basis $A_{1}, \ldots, A_{N}$ of $F$ at $\tau$ with respect to an admissible phase $\phi$ (see $\left.\S 4.5\right)$ is given by:

$$
A_{i}=\widehat{\Gamma}_{F} \operatorname{Ch}\left(E_{i}\right)
$$

In other words, the operator $C^{\prime}$ in Part (3) of Dubrovin's conjecture is given by $C^{\prime}(\alpha)=$ $(2 \pi)^{-\operatorname{dim} F / 2} \widehat{\Gamma}_{F} \alpha$.

Remark 4.6.3. The validity of Gamma Conjecture II does not depend on the choice of $\tau$ and $\phi$. Under deformation of $\tau$ and $\phi$, the marked reflection system $\mathfrak{R e f}(F, \tau, \phi)$ changes by mutations (i.e. defines a continuous path in the space $\mathfrak{M}$ of MRSs) as described in $\S 2.6$ and $\S 4.3$. On the other hand, the braid group acts on full exceptional collections by mutations $(\S 4.4)$. These mutations are compatible in the $K$-group because the Euler pairing $\chi(\cdot, \cdot)$ is identified with the pairing $[\cdot, \cdot)(3.4 .5)$ by Lemma 3.4.6.

Remark 4.6.4. If $F$ satisfies Gamma Conjecture II, the quantum differential equations of $F$ specify a distinguished mutation-equivalence class of (the images in the $K$-group of) full exceptional collections of $F$ given by an asymptotic basis. It is not known in general whether any two given full exceptional collections are connected by mutations and shifts.

Remark 4.6.5. While preparing this paper, we are informed that Dubrovin [19] himself formulated the same conjecture as Gamma Conjecture II.

Proposition 4.6.6. Gamma Conjecture II implies Parts (2) and (3) of Dubrovin's conjecture.

Proof. Recall from Proposition 2.6.4 that the Stokes matrix is given by the pairing $\left[y_{i}, y_{j}\right)$ of asymptotically exponential flat sections. Under Gamma Conjecture II, we have $\left[y_{i}, y_{j}\right)=$ $\left[A_{i}, A_{j}\right)=\chi\left(E_{i}, E_{j}\right)$, where we used the fact that the pairing $[\cdot, \cdot)$ is identified with the Euler pairing (Lemma 3.4.6).

A relationship between Gamma Conjectures I and II is explained as follows.

Proposition 4.6.7. Suppose that a Fano manifold $F$ satisfies Property $\mathcal{O}$ and that the quantum product $\star_{0}$ is semisimple. Suppose also that F satisfies Gamma Conjecture II. If the exceptional object corresponding to the eigenvalue $T$ and an admissible phase $\phi$ with $|\phi|<\pi / 2$ is the structure sheaf $\mathcal{O}$ (or its shift), then $F$ satisfies Gamma Conjecture I.

Proof. Under the assumptions, the flat section $\Phi\left(\widehat{\Gamma}_{F}\right)$ corresponding to $\mathcal{O}$ satisfies the asymptotics $\Phi\left(\widehat{\Gamma}_{F}\right)(z) \sim e^{-T / z} \psi_{0}$ for some $\psi_{0} \in E(T)$, as $z \rightarrow 0$ in the sector $\phi-\frac{\pi}{2}-\epsilon<\arg z<$ $\phi+\frac{\pi}{2}+\epsilon$ (see Proposition 2.5.1). Therefore $\Phi\left(\widehat{\Gamma}_{F}\right)$ belongs to the space $\mathcal{A}(3.3 .2)$. Gamma Conjecture I holds (see Conjecture 3.4.3, Theorem 3.6.8).

4.7. Quantum cohomology central charges. Suppose that $F$ satisfies Gamma Conjecture II and that $\star_{0}$ is semisimple. Let $\psi_{1}, \ldots, \psi_{N}$ be the idempotent basis for $\star_{0}$ and $u_{1}, \ldots, u_{N}$ be the corresponding eigenvalues of $\left(c_{1}(F) \star_{0}\right)$ i.e. $c_{1}(F) \star_{0} \psi_{i}=u_{i} \psi_{i}$. Let $\phi \in \mathbb{R}$ be an admissible phase for $\left\{u_{1}, \ldots, u_{N}\right\}$ and let $E_{1}, \ldots, E_{N}$ be an exceptional collection corresponding (via (4.6.2)) to the asymptotic basis $A_{1}, \ldots, A_{N}$ at $\tau=0$ with respect to the phase $\phi$. Then the quantum cohomology central charges of $E_{1}, \ldots, E_{N}$ (see (3.8.1), (3.8.2)) have the following asymptotics:

$$
Z\left(E_{i}\right) \sim \sqrt{\left(\psi_{i}, \psi_{i}\right)_{F}}(2 \pi z)^{\operatorname{dim} F / 2} e^{-u_{i} / z}
$$

as $z \rightarrow 0$ in the sector $\phi-\frac{\pi}{2}-\epsilon<\arg z<\phi+\frac{\pi}{2}+\epsilon$ for a sufficiently small $\epsilon>0$. This follows from the definition (3.8.1) of $Z\left(E_{i}\right)$ and the asymptotics $\Phi\left(A_{i}\right)(z) \sim e^{-u_{i} / z} \psi_{i} / \sqrt{\left(\psi_{i}, \psi_{i}\right)_{F}}$. See also $\S 3.8$. 


\section{Gamma COnjectures for Projective spaces}

In this section we prove the Gamma Conjectures for projective spaces following the method of Dubrovin [17, Example 4.4]. The Gamma conjectures for projective spaces also follow from the computations in $[44,45,49]$.

Theorem 5.0.1. Gamma Conjectures $I$ and II hold for the projective space $\mathbb{P}=\mathbb{P}^{N-1}$. An asymptotic basis is formed by mutations of the Gamma basis $\widehat{\Gamma}_{\mathbb{P}} \mathrm{Ch}(\mathcal{O}(i))$ associated to Beilinson's exceptional collection $\{\mathcal{O}(i): 0 \leqslant i \leqslant N-1\}$.

Corollary 5.0.2 (Guzzetti [35], Tanabé [60]). Dubrovin's conjecture holds for $\mathbb{P}=\mathbb{P}^{N-1}$.

5.1. Quantum connection of projective spaces. Let $\mathbb{P}$ denote the projective space $\mathbb{P}^{N-1}$. Let $h=c_{1}(\mathcal{O}(1)) \in H^{2}(\mathbb{P})$ denote the hyperplane class. The quantum product by $h$ is given by:

$$
h \star_{0} h^{i}= \begin{cases}h^{i+1} & \text { if } 0 \leqslant i \leqslant N-2 ; \\ 1 & \text { if } i=N-1 .\end{cases}
$$

It follows that the quantum multiplication $\left(c_{1}(\mathbb{P}) \star_{0}\right)=N\left(h \star_{0}\right)$ is a semisimple operator with pairwise distinct eigenvalues. Also Property $\mathcal{O}$ (Definition 3.1.1) holds for $\mathbb{P}$ with $T=N$. Let $\nabla$ be the quantum connection (2.2.1) at $\tau=0$. The differential equation $\nabla f(z)=0$ for a cohomology-valued function $f(z)=\sum_{i=0}^{N-1} f_{i}(z) z^{i-\frac{N-1}{2}} h^{N-1-i}$ reads:

$$
\begin{aligned}
& f_{i}(z)=N^{-i}\left(z \partial_{z}\right)^{i} f_{0}(z) \quad 1 \leqslant i \leqslant N-1, \\
& f_{0}(z)=z^{N} N^{-N}\left(z \partial_{z}\right)^{N} f_{0}(z) .
\end{aligned}
$$

Therefore a flat section $f(z)$ for $\nabla$ is determined by its top-degree component $z^{-\frac{N-1}{2}} f_{0}(z)$ which satisfies the scalar differential equation:

$$
\left(D^{N}-N^{N}(-t)^{N}\right) f_{0}=0
$$

where we set $t:=z^{-1}$ and write $D:=t \partial_{t}=-z \partial_{z}$.

Remark 5.1.2. Equation (5.1.1) is the quantum differential equation of $\mathbb{P}$ with $t$ replaced with $-t$ (see Remark 3.6.5).

5.2. Frobenius solutions and Mellin solutions. Solving the differential equation (5.1.1) by the Frobenius method, we obtain a series solution

$$
\Pi(t ; h):=e^{-N h \log t} \sum_{n=0}^{\infty} \frac{\Gamma(h-n)^{N}}{\Gamma(h)^{N}} t^{N n}
$$

taking values in the ring $\mathbb{C}[h] /\left(h^{N}\right)$. Expanding $\Pi(t ; h)$ in the nilpotent element $h$, we obtain a basis $\left\{\Pi_{k}(t): 0 \leqslant k \leqslant N-1\right\}$ of solutions as follows:

$$
\Pi(t ; h)=\Pi_{0}(t)+\Pi_{1}(t) h+\cdots+\Pi_{N-1}(t) h^{N-1} .
$$

Since $h^{N}=0$, we may identify $h$ with the hyperplane class of $\mathbb{P}$. The basis $\left\{\Pi_{k}(t)\right\}$ yields the fundamental solution $S(z) z^{-\mu} z^{\rho}$ of $\nabla$ near the regular singular point $t=z^{-1}=0$ in Proposition 2.3.1. More precisely, we have:

$$
S(z) z^{-\mu} z^{\rho} h^{N-1-k}=\left(\begin{array}{c}
t^{-\frac{N-1}{2}}\left(-\frac{1}{N} D\right)^{N-1} \Pi_{k}(t) \\
\vdots \\
t^{\frac{N-3}{2}}\left(-\frac{1}{N} D\right) \Pi_{k}(t) \\
t^{\frac{N-1}{2}} \Pi_{k}(t)
\end{array}\right)
$$


where the right-hand side is presented in the basis $\left\{1, h, \ldots, h^{N-1}\right\}$.

Another solution to equation (5.1.1) is given by the Mellin transform of $\Gamma(s)^{N}$ :

$$
\Psi(t):=\frac{1}{2 \pi i} \int_{c-i \infty}^{c+i \infty} \Gamma(s)^{N} t^{-N s} d s
$$

with $c>0$. The integral does not depend on $c>0$. One can easily check that $\Psi(t)$ satisfies equation (5.1.1) by using the identity $s \Gamma(s)=\Gamma(s+1)$. Comparing (5.2.1) and (5.2.3), we can think of $\Psi(t)$ as being obtained from $\Pi(t ; h)$ by multiplying $\Gamma(h)^{N}$ and replacing the discrete sum over $n$ with a "continuous" sum (i.e. integral) over $s$.

Note that we have a standard determination for $\Pi_{k}(t)$ and $\Psi(t)$ along the positive real line by requiring that $\log t \in \mathbb{R}$ and $t^{-N s}=e^{-N s \log t}$ for $t \in \mathbb{R}_{>0}$. We see that $\Psi(t)$ gives rise to a flat section with the smallest asymptotics along the positive real line (see §3.3).

Proposition 5.2.4. The solution $\Psi(t)$ (5.2.3) of (5.1.1) satisfies the asymptotics

$$
\Psi(t) \sim C t^{-\frac{N-1}{2}} e^{-N t}\left(1+O\left(t^{-1}\right)\right)
$$

as $t \rightarrow \infty$ in the sector $-\frac{\pi}{2}<\arg t<\frac{\pi}{2}$, where $C=N^{-1 / 2}(2 \pi)^{(N-1) / 2}$. Write the Gamma class of $\mathbb{P}=\mathbb{P}^{N-1}$ as $\widehat{\Gamma}_{\mathbb{P}}=\Gamma(1+h)^{N}=\sum_{k=0}^{N-1} c_{k} h^{N-1-k}$. Then we have the following connection formula:

$$
\Psi(t)=\sum_{k=0}^{N-1} c_{k} \Pi_{k}(t)=\int_{\mathbb{P}} \widehat{\Gamma}_{\mathbb{P}} \cup \Pi(t ; h)
$$

under the analytic continuation along the positive real line.

The connection formula in this proposition and equation (5.2.2) show that:

$$
S(z) z^{-\mu} z^{\rho} \widehat{\Gamma}_{\mathbb{P}}=\left(\begin{array}{c}
t^{-\frac{N-1}{2}}\left(-\frac{1}{N} D\right)^{N-1} \Psi(t) \\
\vdots \\
t^{\frac{N-3}{2}}\left(-\frac{1}{N} D\right) \Psi(t) \\
t^{\frac{N-1}{2}} \Psi(t)
\end{array}\right) .
$$

This flat section has the smallest asymptotics $\sim e^{-N t}$ as $t \rightarrow+\infty$ (see (3.3.2)). Therefore we conclude (recall Conjecture 3.4.3):

Corollary 5.2.6. Gamma Conjecture I holds for $\mathbb{P}$.

Proof of Proposition 5.2.4. The function $\Psi(t)$ is one of Meijer's $G$-functions [55] and the large $t$ asymptotics here was obtained by Meijer, see equations (22), (23), (24) ibid. Here we follow Dubrovin [17, Example 4.4] and use the method of stationary phase to obtain the asymptotics of $\Psi(t)$. We write

$$
\Psi(t)=\frac{1}{2 \pi i} \int_{c-i \infty}^{c+i \infty} e^{f_{t}(s)} d s
$$

with $f_{t}(s)=N(\log \Gamma(s)-s \log t)$. The integral is approximated by a contribution around the critical point of $f_{t}(s)$. Using Stirling's formula

$$
\log \Gamma(s) \sim\left(s-\frac{1}{2}\right) \log s-s+\frac{1}{2} \log (2 \pi)+O\left(s^{-1}\right)
$$


we find a critical point $s_{0}$ of $f_{t}(s)$ such that $s_{0} \sim t+\frac{1}{2}+O\left(t^{-1}\right)$ as $t \rightarrow \infty$. Then we have

$$
\begin{aligned}
& f_{t}\left(s_{0}\right) \sim-N t+\frac{N}{2} \log (2 \pi / t)+O\left(t^{-1}\right), \\
& f_{t}^{\prime \prime}\left(s_{0}\right) \sim \frac{N}{t}+O\left(t^{-2}\right) .
\end{aligned}
$$

From these we obtain the asymptotics:

$$
\Psi(t) \sim \frac{1}{\sqrt{2 \pi f_{t}^{\prime \prime}\left(s_{0}\right)}} e^{f_{t}\left(s_{0}\right)}=\frac{1}{\sqrt{N}}\left(\frac{2 \pi}{t}\right)^{\frac{N-1}{2}} e^{-N t} .
$$

To show the connection formula, we close the integration contour in (5.2.3) to the left and express $\Psi(t)$ as the sum of residues at $s=0,-1,-2,-3, \ldots$ We have for $n \in \mathbb{Z}_{\geqslant 0}$

$$
\begin{aligned}
\operatorname{Res}_{s=-n} \Gamma(s)^{N} t^{-N s} d s & =\operatorname{Res}_{h=0} \Gamma(h-n)^{N} t^{N n-N h} d h \\
& =\int_{\mathbb{P}} \Gamma(1+h)^{N} \frac{\Gamma(h-n)^{N}}{\Gamma(h)^{N}} t^{N n-N h} .
\end{aligned}
$$

In the second line we used the fact that $\Gamma(1+h) / \Gamma(h)=h$ and $\int_{\mathbb{P}} g(h)=\operatorname{Res}_{h=0}\left(g(h) / h^{N}\right) d h$ for any $g(h) \in \mathbb{C} \llbracket h \rrbracket$. Therefore we have

$$
\Psi(t)=\sum_{n=0}^{\infty} \operatorname{Res}_{s=-n} \Gamma(s)^{N} t^{-N s} d s=\int_{\mathbb{P}} \widehat{\Gamma}_{\mathbb{P}} \cup \Pi(t ; h)
$$

as required.

5.3. Monodromy transformation and mutation. We use monodromy transformation and mutation to deduce Gamma Conjecture II for $\mathbb{P}$ from the truth of Gamma Conjecture I for $\mathbb{P}$.

The differential equation (5.1.1) is invariant under the rotation $t \rightarrow e^{2 \pi \mathrm{i} / N} t$. Therefore the functions $\Psi^{(j)}(t)=\Psi\left(e^{-2 \pi i j / N} t\right), j \in \mathbb{Z}$ are also solutions to (5.1.1). By changing co-ordinates $t \rightarrow e^{-2 \pi \mathrm{i} j / N} t$ in Proposition 5.2.4, we find that $\Psi^{(j)}$ satisfies the asymptotic condition

$$
\Psi^{(j)}(t) \sim C_{j} t^{-\frac{N-1}{2}} e^{-N \zeta^{-j}}
$$

in the sector $-\frac{\pi}{2}+\frac{2 \pi j}{N}<\arg t<\frac{\pi}{2}+\frac{2 \pi j}{N}$ where $\zeta=\exp (2 \pi \mathrm{i} / N)$ and $C_{j}=C e^{(N-1) \pi \mathrm{i} j / N}$. Using $\Pi\left(e^{-2 \pi \mathrm{i} j / N} t ; h\right)=\operatorname{Ch}(\mathcal{O}(i)) \cup \Pi(t ; h)$, we also find the connection formula

$$
\Psi^{(j)}(t)=\int_{\mathbb{P}} \widehat{\Gamma}_{\mathbb{P}} \operatorname{Ch}(\mathcal{O}(j)) \cup \Pi(t ; h) .
$$

Let $y_{j}(z)$ be the flat section corresponding to $\Psi^{(j)}$ (cf. (5.2.5)):

$$
y_{j}(z):=(2 \pi)^{-\frac{N-1}{2}}\left(\begin{array}{c}
t^{-\frac{N-1}{2}}\left(-\frac{1}{N} D\right)^{N-1} \Psi^{(j)}(t) \\
\vdots \\
t^{\frac{N-3}{2}}\left(-\frac{1}{N} D\right) \Psi^{(j)}(t) \\
t^{\frac{N-1}{2}} \Psi^{(j)}(t)
\end{array}\right) .
$$

The above connection formula and (5.2.2) show that

$$
y_{j}(z)=(2 \pi)^{-\frac{N-1}{2}} S(z) z^{-\mu} z^{\rho}\left(\widehat{\Gamma}_{\mathbb{P}} \operatorname{Ch}(\mathcal{O}(i))\right)=\Phi\left(\widehat{\Gamma}_{\mathbb{P}} \operatorname{Ch}(\mathcal{O}(i))\right)
$$

where we recall that $\Phi$ was defined in (3.4.1). Since the sectors where the asymptotics (5.3.1) of $\Psi^{(j)}$ hold depend on $j$, the flat sections $y_{j}(z)$ do not quite form the asymptotically exponential 
fundamental solution in the sense of Proposition 2.5.1. We will see however that they give it after a sequence of mutations.

Recall from Proposition 3.4.8 that the flat section $y_{0}(z)$ with the smallest asymptotics (along $\mathbb{R}_{>0}$ ) can be written as the Laplace integral:

$$
y_{0}(z)=\frac{1}{z} \int_{T}^{\infty} \varphi(\lambda) e^{-\lambda / z} d \lambda
$$

for some $\widehat{\nabla}$-flat section $\varphi(\lambda)$ holomorphic near $\lambda=T(=N)$. Recall that $\hat{\nabla}$ is the Laplace dual (2.5.3) of $\nabla$ at $\tau=0$. This integral representation is valid when $\operatorname{Re}(z)>0$. We have

$$
\begin{aligned}
y_{j}(z) & =e^{2 \pi \mathrm{i} j \mu / N} y_{0}\left(e^{2 \pi \mathrm{i} j / N} z\right)=\frac{\zeta^{-j}}{z} \int_{T}^{\infty} e^{2 \pi \mathrm{i} j \mu / N} \varphi(\lambda) e^{-\zeta^{-j} \lambda / z} d \lambda \\
& =\int_{T \zeta^{-j}+\mathbb{R}_{\geqslant 0} \zeta^{-j}} e^{2 \pi \mathrm{i} j \mu / N} \varphi\left(\zeta^{j} \lambda\right) e^{-\lambda / z} d \lambda .
\end{aligned}
$$

Set $\varphi_{j}(\lambda)=e^{2 \pi \mathrm{i} j \mu / N} \varphi\left(\zeta^{j} \lambda\right)$. Then $\varphi_{j}(\lambda)$ is holomorphic near $\lambda=\zeta^{-j} T$ and is flat for $\hat{\nabla}$. The latter fact follows easily from (3.1.4). Thus we obtain an integral representation of $y_{j}(z)$ :

$$
y_{j}(z)=\frac{1}{z} \int_{T \zeta^{-j}+\mathbb{R}_{\geqslant 0} \zeta^{-j}} \varphi_{j}(\lambda) e^{-\lambda / z} d \lambda
$$

which is valid when $-\frac{\pi}{2}-\frac{2 \pi j}{N}<\arg z<\frac{\pi}{2}-\frac{2 \pi j}{N}$. By bending the radial integration path, we can analytically continue $y_{j}(z)$ for arbitrary $\arg z$. When $\arg z$ is close to zero, for example, we bend the paths as shown in Figure 9 . Here we choose a range $\left[j_{0}, j_{0}+N-1\right]$ of length $N$ and consider a system of integration paths for $y_{j}(z), j \in\left[j_{0}, j_{0}+N-1\right]$ when $\arg z$ is close to zero.

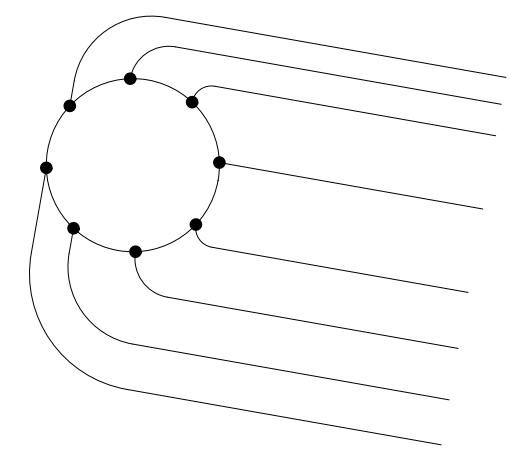

Figure 9. Bent paths starting from $T \zeta^{-j}, j=-3, \cdots, 4(N=8)$.

We can construct an asymptotically exponential fundamental solution in the sense of Proposition 2.5.1 by straightening the paths (see Figure 10). Note that $\lim _{z \rightarrow+0} e^{T / z} y_{0}(z)=\varphi(T)$ is a $T$-eigenvector of $\left(c_{1}(\mathbb{P}) \star_{0}\right)$ of unit length by Proposition 3.4.8. Therefore $\varphi_{j}\left(\zeta^{-j} T\right)=$ $e^{2 \pi \mathrm{i} j \mu / N} \varphi(T)$ is also of unit length. It is a $\zeta^{-j} T$-eigenvector of $\left(c_{1}(\mathbb{P}) \star_{0}\right)$ by (3.1.4). Therefore $\varphi_{j}\left(\zeta^{-j} T\right), j \in\left[j_{0}, j_{0}+N-1\right]$ form a normalized idempotent basis for $\star_{0}$. Let $\phi$ be an admissible phase for the spectrum of $\left(c_{1}(\mathbb{P}) \star_{0}\right)$ which is close to zero. By the discussion in 


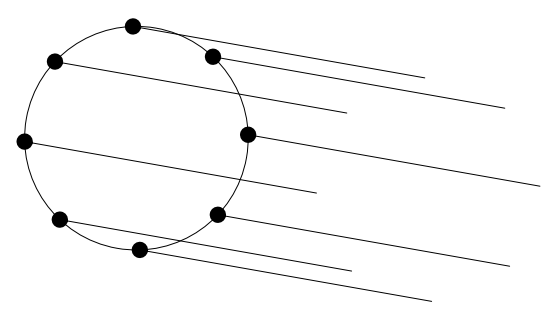

Figure 10. Straightened paths in the admissible direction $e^{\mathrm{i} \phi}$.

$\S 2.5$, the $\nabla$-flat sections

$$
x_{j}(z)=\frac{1}{z} \int_{T \zeta^{-j}+\mathbb{R}_{\geqslant 0} e^{\mathrm{i} \phi}} \varphi_{j}(\lambda) e^{-\lambda / z} d \lambda \sim e^{-\zeta^{-j} T / z} \varphi_{j}\left(\zeta^{-j} T\right)
$$

with $j \in\left[j_{0}, j_{0}+N-1\right]$ give the asymptotically exponential fundamental solution associated to $e^{\mathbf{i} \phi}$. By the argument in $\S 2.6$, the two bases $\left\{y_{j}\right\}$ and $\left\{x_{j}\right\}$ are related by a sequence of mutations. Because $\left\{y_{j}\right\}$ corresponds to the Beilinson collection $\{\mathcal{O}(j)\}$ (see (5.3.2)), $\left\{x_{j}\right\}$ corresponds to a mutation of $\{\mathcal{O}(j)\}$ (see $\S 2.6$ and Remark 4.6.3). This shows that the asymptotic basis at $\tau=0$ with respect to phase $\phi$ is given by a mutation of $\widehat{\Gamma}_{\mathbb{P}} \mathrm{Ch}(\mathcal{O}(j))$, $j_{0} \leqslant j \leqslant j_{0}+N-1$.

The proof of Theorem 5.0.1 is now complete.

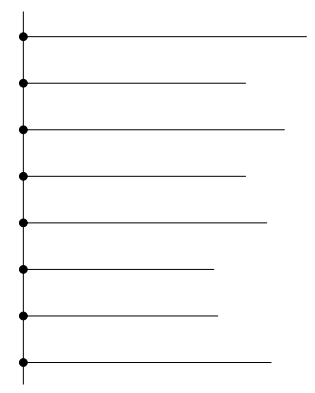

FiguRE 11. A system of paths after isomonodromic deformation.

Remark 5.3.3. In $\S 2.6$ and $\S 4.3$, we considered mutations for straight paths. Although our paths in Figure 9 are not straight, we can make them straight after isomonodromic deformation (see Figure 11). We can therefore apply our mutation argument to such straight paths. When the eigenvalues of $E \star_{\tau}$ align as in Figure 11, the corresponding flat sections $y_{j}$ form an asymptotically exponential fundamental solution and the Beilinson collection gives the asymptotic basis $\left\{\widehat{\Gamma}_{\mathbb{P}} \mathrm{Ch}(\mathcal{O}(j))\right\}$ at such a point $\tau$. 


\section{Gamma conjectures for Grassmannians}

We derive Gamma Conjectures for Grassmannians of type A from the truth of Gamma Conjectures for projective spaces. Let $\mathbb{G}=G(r, N)$ denote the Grassmannian of $r$-dimensional subspaces of $\mathbb{C}^{N}$ and let $\mathbb{P}=\mathbb{P}^{N-1}=G(1, N)$ denote the projective space of dimension $N-1$.

6.1. Statement. Let $S^{\nu}$ denote the Schur functor for a partition $\nu=\left(\nu_{1} \geqslant \nu_{2} \geqslant \cdots \geqslant \nu_{r}\right)$. Denote by $V$ the tautological bundle ${ }^{9}$ over $\mathbb{G}=G(r, N)$. Kapranov [47, 48] showed that the vector bundles $S^{\nu} V^{*}$ form a full exceptional collection of $\mathcal{D}_{\text {coh }}^{b}(\mathbb{G})$, where $\nu$ ranges over all partitions such that the corresponding Young diagrams are contained in an $r \times(N-r)$ rectangle, i.e. $\nu_{i} \leqslant N-r$ for all $i$.

Theorem 6.1.1. Gamma Conjectures I and II hold for Grassmannians $\mathbb{G}$. An asymptotic basis of $\mathbb{G}$ is formed by mutations of the Gamma basis $\widehat{\Gamma}_{\mathbb{G}} \mathrm{Ch}\left(S^{\nu} V^{*}\right)$ associated to Kapranov's exceptional collection $\left\{S^{\nu} V^{*}: \nu\right.$ is contained in an $r \times(N-r)$ rectangle $\}$.

Corollary 6.1.2 (Ueda [64]). Dubrovin's Conjecture holds for $\mathbb{G}$.

The proof of Gamma Conjecture II for $\mathbb{G}$ will be completed in $\S 6.6$; the proof of Gamma Conjecture I for $\mathbb{G}$ will be completed in $\S 6.7$.

6.2. Quantum Pieri and quantum Satake. For background material on classical cohomology rings of Grassmannians, we refer the reader to [21]. Let $x_{1}, \ldots, x_{r}$ be the Chern roots of the dual $V^{*}$ of the tautological bundle over $\mathbb{G}=G(r, N)$. Every cohomology class of $\mathbb{G}$ can be written as a symmetric polynomial in $x_{1}, \ldots, x_{r}$. We have:

$$
H^{\bullet}(\mathbb{G}) \cong \mathbb{C}\left[x_{1}, \ldots, x_{r}\right]^{\mathfrak{S}_{r}} /\left\langle h_{N-r+1}, \ldots, h_{N}\right\rangle
$$

where $h_{i}=h_{i}\left(x_{1}, \ldots, x_{r}\right)$ is the $i$ th complete symmetric polynomial of $x_{1}, \ldots, x_{r}$. An additive basis of $H^{\bullet}(\mathbb{G})$ is given by the Schur polynomials ${ }^{10}$

$$
\sigma_{\lambda}=\sigma_{\lambda}\left(x_{1}, \ldots, x_{r}\right)=\frac{\operatorname{det}\left(x_{i}^{\lambda_{j}+r-j}\right)_{1 \leqslant i, j \leqslant r}}{\operatorname{det}\left(x_{i}^{r-j}\right)_{1 \leqslant i, j \leqslant r}}
$$

with partition $\lambda$ in an $r \times(N-r)$ rectangle. The class $\sigma_{\lambda}$ is Poincaré dual to the Schubert cycle $\Omega_{\lambda}$ and $\operatorname{deg} \sigma_{\lambda}=2|\lambda|=2 \sum_{i=1}^{r} \lambda_{i}$. For $1 \leqslant k \leqslant N-r$, we write $\sigma_{k}=h_{k}\left(x_{1}, \ldots, x_{r}\right)=c_{k}(Q)$ for the Schubert class corresponding to the partition $(k \geqslant 0 \geqslant \cdots \geqslant 0)$. Here $Q$ is the universal quotient bundle. They are called the special Schubert classes. The first Chern class is given by $c_{1}(\mathbb{G})=N \sigma_{1}$. The classical Pieri formula describes the multiplication by the special Schubert classes:

$$
\sigma_{k} \cup \sigma_{\lambda}=\sum \sigma_{\nu}
$$

where the sum ranges over all partitions $\nu=\left(\nu_{1} \geqslant \cdots \geqslant \nu_{r}\right)$ in an $r \times(N-r)$ rectangle such that $|\nu|=|\lambda|+k$ and $\nu_{1} \geqslant \lambda_{1} \geqslant \nu_{2} \geqslant \lambda_{2} \geqslant \cdots \geqslant \nu_{r} \geqslant \lambda_{r}$. Bertram's quantum Pieri formula gives the quantum multiplication by special Schubert classes:

Proposition 6.2.3 (Quantum Pieri [8], see also [10, 22]). We have

$$
\sigma_{k} \star_{\sigma_{1} \log q} \sigma_{\lambda}=\sum \sigma_{\nu}+q \sum \sigma_{\mu}
$$

where the first sum is the same as the classical Pieri formula (6.2.2) and the second sum ranges over all $\mu$ in an $r \times(N-r)$ rectangles such that $|\mu|=|\lambda|+k-N$ and $\lambda_{1}-1 \geqslant \mu_{1} \geqslant$ $\lambda_{2}-1 \geqslant \mu_{2} \geqslant \cdots \geqslant \lambda_{r}-1 \geqslant \mu_{r} \geqslant 0$.

\footnotetext{
${ }^{9}$ The dual $V^{*}$ can be identified with the universal quotient bundle on the dual Grassmannian $G(N-r, N)$.

${ }^{10}$ The Satake identification $H^{\bullet}(\mathbb{G}) \cong \wedge^{r} H^{\bullet}(\mathbb{P})$ is also indicated by this expression.
} 
From the quantum Pieri formula, one can deduce that the quantum connection of $\mathbb{G}$ is the wedge power of the quantum connection of $\mathbb{P}$. This is an instance of the quantum Satake principle of Golyshev-Manivel [32]. The geometric Satake correspondence of Ginzburg [26] implies that the intersection cohomology of an affine Schubert variety $X_{\lambda}$ in the affine Grassmannian of a complex Lie group $G$ becomes an irreducible representation of the Langlands dual Lie group $G^{L}$. Our target spaces $\mathbb{P}, \mathbb{G}$ arise as certain minuscule Schubert varieties in the affine Grassmannian of $G L_{N}(\mathbb{C})$. Therefore the cohomology groups of $\mathbb{P}$ and $\mathbb{G}$ are representations of $G L_{N}(\mathbb{C}): H^{\bullet}(\mathbb{P})$ is the standard representation of $G L\left(H^{\bullet}(\mathbb{P})\right) \cong G L_{N}(\mathbb{C})$ and $H^{\bullet}(\mathbb{G})$ is the $r$-th wedge power of the standard representation. In fact, by the 'take the span' rational map $\mathbb{P} \times \cdots \times \mathbb{P}(r$ factors $) \rightarrow \mathbb{G}$, we obtain the Satake identification

$$
\text { Sat: } \wedge^{r} H^{\bullet}(\mathbb{P}) \stackrel{\cong}{\rightrightarrows} H^{\bullet}(\mathbb{G}), \quad \sigma_{\lambda_{1}+r-1} \wedge \sigma_{\lambda_{2}+r-2} \wedge \cdots \wedge \sigma_{\lambda_{r}} \longmapsto \sigma_{\lambda}
$$

where $\sigma_{\lambda_{i}+r-i}=\left(\sigma_{1}\right)^{\lambda_{i}+r-i}$ is the special Schubert class of $\mathbb{P}=G(1, N)$. The Satake identification endows $H^{\bullet}(\mathbb{G})$ with the structure of a $\mathfrak{g l}\left(H^{\bullet}(\mathbb{P})\right)$-module.

Proposition 6.2.5 $([9$, Theorem 2.5$],[32, \S 1.6])$. The quantum product $\left(c_{1}(\mathbb{G}) \star_{0}\right)$ on $H^{\bullet}(\mathbb{G})$ coincides, upon the Satake identification Sat, with the action of $\left(c_{1}(\mathbb{P}) \star_{\pi \mathfrak{i}(r-1) \sigma_{1}}\right) \in \mathfrak{g l}\left(H^{\bullet}(\mathbb{P})\right)$ on the $r$-th wedge representation $\wedge^{r} H^{\bullet}(\mathbb{P})$.

Proof. This follows from quantum Pieri in Proposition 6.2.3. Recall that $c_{1}(\mathbb{P})=N \sigma_{1}$ and $c_{1}(\mathbb{G})=N \sigma_{1}$. Thus it suffices to examine the quantum product by $\sigma_{1}$. The action of $\left(\sigma_{1} \star_{\pi \mathrm{i}(r-1) \sigma_{1}}\right)$ on the basis element $\sigma_{\lambda_{1}+r-1} \wedge \sigma_{\lambda_{2}+r-2} \wedge \cdots \wedge \sigma_{\lambda_{r}}$ of the $r$-th wedge $\wedge^{r} H^{\bullet}(\mathbb{P})$ is given by

$$
\begin{aligned}
& \sum_{i=1}^{r} \sigma_{\lambda_{1}+r-1} \wedge \cdots \wedge\left(\sigma_{1} \star_{\pi \mathrm{i}(r-1) \sigma_{1}} \sigma_{\lambda_{i}+r-i}\right) \wedge \cdots \wedge \sigma_{\lambda_{r}} \\
= & \left(\sum_{i=1}^{r} \sigma_{\lambda_{1}+r-1} \wedge \cdots \wedge \sigma_{\lambda_{i}+r-i+1} \wedge \cdots \wedge \sigma_{\lambda_{r}}\right)+(-1)^{r-1} \delta_{N-1, \lambda_{1}+r-1} \sigma_{0} \wedge \sigma_{\lambda_{2}+r-2} \wedge \cdots \wedge \sigma_{\lambda_{r}}
\end{aligned}
$$

Under the Satake identification, the first term corresponds to the classical Pieri formula and the second term corresponds to the quantum correction. The sign $(-1)^{r-1}$ cancels the sign coming from the permutation of $\sigma_{0}$ and $\sigma_{\lambda_{2}+r-2} \wedge \cdots \wedge \sigma_{\lambda_{r}}$.

Remark 6.2.6 ([32]). More generally, the action of the power sum $\left(x_{1}^{k}+\cdots+x_{r}^{k}\right) \star_{0}$ on $H^{\bullet}(\mathbb{G})$ coincides, upon the Satake identification with the action of $\left(\sigma_{k^{\star}}(r-1) \pi \mathrm{i} \sigma_{1}\right) \in \mathfrak{g l}\left(H^{\bullet}(\mathbb{P})\right)$ on the $r$-th wedge representation $\wedge^{r} H^{\bullet}(\mathbb{P})$, cf. Theorem 6.3.1.

We have a similar result for the grading operator. The following lemma together with Proposition 6.2.5 implies that the quantum connection of $\mathbb{G}$ is the wedge product of the quantum connection of $\mathbb{P}$.

Lemma 6.2.7. Let $\mu^{\mathbb{P}}$ and $\mu^{\mathbb{G}}$ be the grading operators of $\mathbb{P}$ and $\mathbb{G}$ respectively (see $\left.\S 2.2\right)$. The action of $\mu^{\mathbb{G}}$ coincides, upon the Satake identification with the action of $\mu^{\mathbb{P}} \in \mathfrak{g l}\left(H^{\bullet}(\mathbb{P})\right)$ on the $r$-th wedge representation $\wedge^{r} H^{\bullet}(\mathbb{P})$.

Proof. This is immediate from the definition: we use $\operatorname{dim} \mathbb{G}+r(r-1)=r \operatorname{dim} \mathbb{P}$.

The Satake identification also respects the Poincaré pairing up to sign. The Poincaré pairing on $H^{\bullet}(\mathbb{P})$ induces the pairing on the $r$-th wedge $\wedge^{r} H^{\bullet}(\mathbb{P})$ :

$$
\left(\alpha_{1} \wedge \cdots \wedge \alpha_{r}, \beta_{1} \wedge \cdots \wedge \beta_{r}\right)_{\wedge \mathbb{P}}:=\operatorname{det}\left(\left(\alpha_{i}, \beta_{j}\right)_{\mathbb{P}}\right)_{1 \leqslant i, j \leqslant r}
$$


Lemma 6.2.8. We have $(\alpha, \beta)_{\wedge \mathbb{P}}=(-1)^{r(r-1) / 2}(\operatorname{Sat}(\alpha) \text {, } \operatorname{Sat}(\beta))_{\mathbb{G}}$ for $\alpha, \beta \in \wedge^{r} H^{\bullet}(\mathbb{P})$.

Proof. For two partitions $\lambda, \mu$ in an $r \times(N-r)$ rectangle, we have $\left(\sigma_{\lambda}, \sigma_{\mu}\right)_{\mathbb{G}}=1$ if $\lambda_{i}+\mu_{r-i+1}=$ $N-r$ for all $i$, and $\left(\sigma_{\lambda}, \sigma_{\mu}\right)_{\mathbb{G}}=0$ otherwise. On the other hand, if $\lambda_{i}+\mu_{r-i+1}=N-r$ for all $i$, we have

$$
\begin{aligned}
\left(\sigma_{\lambda_{1}+r-1} \wedge \cdots \wedge \sigma_{\lambda_{r}},\right. & \left.\sigma_{\mu_{1}+r-1} \wedge \cdots \wedge \sigma_{\mu_{r}}\right)_{\wedge \mathbb{P}} \\
& =\left(\sigma_{\lambda_{1}+r-1} \wedge \cdots \wedge \sigma_{\lambda_{r}}, \sigma_{N-1-\lambda_{r}} \wedge \cdots \wedge \sigma_{N-r-\lambda_{1}}\right)_{\wedge \mathbb{P}}=(-1)^{r(r-1) / 2} .
\end{aligned}
$$

Otherwise, the pairing can be easily seen to be zero.

Remark 6.2.9. Proposition 6.2.5 implies the well-known formula that the spectrum of $\left(c_{1}(\mathbb{G}) \star_{0}\right)$ consists of sums of $r$ distinct eigenvalues of $\left(c_{1}(\mathbb{P}) \star_{(r-1) \pi \mathrm{i} \sigma_{1}}\right)$, i.e.

$$
\operatorname{Spec}\left(c_{1}(\mathbb{G}) \star_{0}\right)=\left\{N e^{(r-1) \pi \mathrm{i} / N}\left(\zeta_{N}^{i_{1}}+\cdots+\zeta_{N}^{i_{r}}\right): 0 \leqslant i_{1}<i_{2}<\cdots<i_{r} \leqslant N-1\right\}
$$

where $\zeta_{N}=e^{2 \pi i / N}$. In particular $\mathbb{G}$ satisfies Property $\mathcal{O}$ (Definition 3.1 .1 ) with $T=$ $N \sin (\pi r / N) / \sin (\pi / N)$. The quantum product $\left(c_{1}(\mathbb{G}) \star_{0}\right)$ has pairwise distinct eigenvalues if $k !$ and $N$ are coprime, where $k=\min (r, N-r)$.

6.3. The wedge product of the big quantum connection of $\mathbb{P}$. The quantum Satake principle implies that the quantum connection for $\mathbb{G}($ at $\tau=0)$ is the $r$-th wedge product of the quantum connection for $\mathbb{P}($ at $\tau=(r-1) \pi i)$. By using the abelian/non-abelian correspondence of Bertram-Ciocan-Fontanine-Kim-Sabbah $[9,14,50]$, we observe that this is also true for the isomonodromic deformation corresponding to the big quantum cohomology of $\mathbb{P}$ and $\mathbb{G}$. When the eigenvalues of $\left(c_{1}(\mathbb{G}) \star_{0}\right)$ are pairwise distinct, this can be also deduced from the quantum Pieri (Proposition 6.2.3) and Dubrovin's reconstruction theorem [16]; however it is not always true that $\left(c_{1}(\mathbb{G}) \star_{0}\right)$ has pairwise distinct eigenvalues (see Remark 6.2.9).

The quantum product $\star_{(r-1) \pi \mathrm{i} \sigma_{1}}$ of $\mathbb{P}=\mathbb{P}^{N-1}$ is semisimple and $\left(c_{1}(\mathbb{P}) \star_{(r-1) \pi \mathrm{i} \sigma_{1}}\right)$ has pairwise distinct eigenvalues $\mathbf{u}^{\circ}=\left\{N e^{\pi \mathrm{i}(r-1) / N} e^{2 \pi \mathrm{i} k / N}: 0 \leqslant k \leqslant N-1\right\}$. As explained in $\S 2.7$, the quantum connection of $\mathbb{P}$ has an isomonodromic deformation over the universal cover $C_{N}(\mathbb{C})^{\sim}$ of the configuration space $(2.7 .1)$ of distinct $N$ points in $\mathbb{C}$. Let $\nabla^{\mathbb{P}}$ be the connection on the trivial bundle $H^{\bullet}(\mathbb{P}) \times\left(C_{N}(\mathbb{C})^{\sim} \times \mathbb{P}^{1}\right) \rightarrow\left(C_{N}(\mathbb{C})^{\sim} \times \mathbb{P}^{1}\right)$ which gives the isomonodromic deformation (see Proposition 2.7.2). Here the germ $\left(C_{N}(\mathbb{C})^{\sim}, \mathbf{u}^{\circ}\right)$ at $\mathbf{u}^{\circ}$ is identified with the germ $\left(H^{\bullet}(\mathbb{P}),(r-1) \pi \mathbf{i} \sigma_{1}\right)$ by the eigenvalues of $\left(E \star_{\tau}\right)$ and $\nabla^{\mathbb{P}}$ is identified with the big quantum connection (2.2.3) of $\mathbb{P}$ near $\mathbf{u}^{\circ}$. Consider the $r$-th wedge product of this bundle:

$$
\left(\wedge^{r} H^{\bullet}(\mathbb{P})\right) \times\left(C_{N}(\mathbb{C})^{\sim} \times \mathbb{P}^{1}\right) \rightarrow\left(C_{N}(\mathbb{C})^{\sim} \times \mathbb{P}^{1}\right)
$$

equipped with the meromorphic flat connection $\nabla^{\wedge \mathbb{P}}$ :

$$
\nabla^{\wedge \mathbb{P}}\left(\xi_{1} \wedge \xi_{2} \wedge \cdots \wedge \xi_{r}\right):=\sum_{i=1}^{r} \xi_{1} \wedge \cdots \wedge\left(\nabla^{\mathbb{P}} \xi_{i}\right) \wedge \cdots \wedge \xi_{r} .
$$

Theorem 6.3.1. There exists an embedding $f:\left(H^{\bullet}(\mathbb{P}),(r-1) \pi \mathbf{i} \sigma\right) \cong\left(C_{N}(\mathbb{C})^{\sim}, \mathbf{u}^{\circ}\right) \rightarrow$ $\left(H^{\bullet}(\mathbb{G}), 0\right)$ between germs of complex manifolds such that the big quantum connection $\nabla^{\mathbb{G}}$ of $\mathbb{G}$ pulls back (via $f$ ) to the $r$-th wedge $\nabla^{\wedge \mathbb{P}}$ of the big quantum connection of $\mathbb{P}$ under the Satake identification Sat (6.2.4). More precisely, the bundle map

$$
\begin{aligned}
\wedge^{r} H^{\bullet}(\mathbb{P}) \times\left(C_{N}(\mathbb{C})^{\sim} \times \mathbb{P}^{1}\right) & \longrightarrow H^{\bullet}(\mathbb{G}) \times\left(C_{N}(\mathbb{C})^{\sim} \times \mathbb{P}^{1}\right) \\
(\alpha,(\mathbf{u}, z)) & \longmapsto\left(i^{r(r-1) / 2} \operatorname{Sat}(\alpha),(\mathbf{u}, z)\right)
\end{aligned}
$$

intertwines the connections $\nabla^{\wedge \mathbb{P}}, f^{*} \nabla^{\mathbb{G}}$ and the pairings $(\cdot, \cdot)_{\wedge \mathbb{P}},(\cdot, \cdot)_{\mathbb{G}}$. 
Proof. The proposition follows by unpacking the definition of the "alternate product of Frobenius manifolds" in [50]. The key ingredient is a universal property of the universal deformation (unfolding) of meromorphic connections due to Hertling and Manin [37]. The argument is straightforward, but technical. We recommend the reader unfamiliar with Hertling-Manin's universal deformation to begin by reading $[50, \S 1]$.

We first review the construction of the alternate product for the big quantum cohomology Frobenius manifold of $\mathbb{P}$. A pre-Saito structure $[50, \S 1.1]$ with base $M$ is a meromorphic flat connection $\nabla$ on a trivial vector bundle $E^{0} \times\left(M \times \mathbb{P}^{1}\right) \rightarrow M \times \mathbb{P}^{1}$ of the form:

$$
\nabla=d+\frac{1}{z} \mathcal{C}+\left(-\frac{1}{z} U+V\right) \frac{d z}{z}
$$

for some $\mathcal{C} \in \operatorname{End}\left(E^{0}\right) \otimes \Omega_{M}^{1}$ and $U, V \in \operatorname{End}\left(E^{0}\right) \otimes \mathcal{O}_{M}$ (it follows from the flatness of $\nabla$ that $V$ is constant). Here $E^{0}$ is a finite dimensional complex vector space and the base space $M$ is a complex manifold. The big quantum connection (2.2.3) is an example of a pre-Saito structure. Consider the quantum connection of $\mathbb{P}$ restricted to $H^{2}(\mathbb{P}) \times \mathbb{P}^{1}$. This gives a pre-Saito structure. The external tensor product of this pre-Saito structure yields a pre-Saito structure $\nabla^{\times r}$ on the bundle:

$$
\otimes^{r} H^{\bullet}(\mathbb{P}) \times\left(\left(H^{2}(\mathbb{P})\right)^{r} \times \mathbb{P}^{1}\right) \rightarrow\left(H^{2}(\mathbb{P})\right)^{r} \times \mathbb{P}^{1} .
$$

We choose a base point $t^{\circ} \in H^{2}(\mathbb{P})$. Let $\Delta: H^{\bullet}(\mathbb{P}) \rightarrow\left(H^{\bullet}(\mathbb{P})\right)^{r}$ denote the diagonal map $\Delta(\tau)=(\tau, \ldots, \tau)$. For notational convenience, for a point $x$ on a manifold $M$, we write $M_{x}$ for the germ $(M, x)$ of $M$ at $x$. We restrict the pre-Saito structure $\nabla^{\times r}$ to the germ $\left(H^{2}(\mathbb{P})\right)_{\Delta\left(t^{\circ}\right)}^{r}$. By Hertling-Manin's reconstruction theorem [37], [50, Corollary 1.7] and [50, Lemma 2.1], the pre-Saito structure $\nabla^{\times r}$ admits a universal deformation over the base $\otimes^{r} H^{\bullet}(\mathbb{P})$. Here we embed the base $\left(H^{2}(\mathbb{P})\right)_{\Delta\left(t^{\circ}\right)}^{r}$ into $\otimes^{r} H^{\bullet}(\mathbb{P})_{\chi_{1}\left(\Delta\left(t^{\circ}\right)\right)}$ by the map

$$
\chi_{1}:\left(H^{2}(\mathbb{P})\right)^{r} \hookrightarrow \otimes^{r} H^{\bullet}(\mathbb{P}), \quad \chi_{1}\left(\tau_{1}, \ldots, \tau_{r}\right)=\sum_{i=1}^{r} 1 \otimes \cdots \otimes 1 \otimes \stackrel{i \text { th }}{\tau_{i}} \otimes 1 \otimes \cdots \otimes 1
$$

and the universal deformation is a pre-Saito structure $\nabla^{\otimes r}$ on the bundle

$$
\otimes^{r} H^{\bullet}(\mathbb{P}) \times\left(\otimes^{r} H^{\bullet}(\mathbb{P})_{\chi_{1}\left(\Delta\left(t^{\circ}\right)\right)} \times \mathbb{P}^{1}\right) \rightarrow \otimes^{r} H^{\bullet}(\mathbb{P})_{\chi_{1}\left(\Delta\left(t^{\circ}\right)\right)} \times \mathbb{P}^{1}
$$

such that $\chi_{1}^{*} \nabla^{\otimes r}=\nabla^{\times r}$. The construction of $\nabla^{\otimes r}$ is given in [50, 2.2, p.234]; the embedding $\chi_{1}$ is a primitive of the infinitesimal period mapping (see $[50, \S 1.2]$ ) of the pre-Saito structure $\nabla^{\times r}$ attached to $1 \otimes 1 \otimes \cdots \otimes 1 \in \otimes^{r} H^{\bullet}(\mathbb{P})$. Geometrically this is the big quantum connection of $\mathbb{P} \times \cdots \times \mathbb{P}$ ( $r$ factors). The pre-Saito structure $\nabla^{\otimes r}$ is equivariant with respect to the natural $W:=\mathfrak{S}_{r}$-action. We restrict the pre-Saito structure $\nabla^{\otimes r}$ to the $W$-invariant base $\operatorname{Sym}^{r} H^{\bullet}(\mathbb{P}) \subset \otimes^{r} H^{\bullet}(\mathbb{P})$; fibers of the restriction are representations of $W$. By taking the anti-symmetric part, we obtain a pre-Saito structure $\nabla^{\mathrm{Sym}}$ on the bundle

$$
\wedge^{r} H^{\bullet}(\mathbb{P}) \times\left(\operatorname{Sym}^{r} H^{\bullet}(\mathbb{P})_{\chi_{1}\left(\Delta\left(t^{\circ}\right)\right)} \times \mathbb{P}^{1}\right) \rightarrow \operatorname{Sym}^{r} H^{\bullet}(\mathbb{P})_{\chi_{1}\left(\Delta\left(t^{\circ}\right)\right)} \times \mathbb{P}^{1} .
$$

Here the superscript 'Sym' of $\nabla^{\mathrm{Sym}}$ signifies the base space, not the fiber. We further restrict this pre-Saito structure to the subspace $\operatorname{Elem}^{r} H^{\bullet}(\mathbb{P}) \subset \operatorname{Sym}^{r} H^{\bullet}(\mathbb{P})$ spanned by "elementary symmetric" vectors

$$
\operatorname{Elem}^{r} H^{\bullet}(\mathbb{P})=\bigoplus_{k=1}^{r} \mathbb{C} \sum_{\substack{i_{1}, \ldots, i_{r} \in\{0,1\} \\ \sum_{a=1}^{r} i_{a}=k}} \sigma_{i_{1}} \otimes \cdots \otimes \sigma_{i_{r}} \subset \operatorname{Sym}^{r} H^{\bullet}(\mathbb{P})
$$


which contains $\chi_{1}\left(\Delta\left(H^{2}(X)\right)\right.$. By Hertling-Manin's reconstruction theorem and [50, Lemma $2.9]$, we obtain a universal deformation of the pre-Saito structure $\left.\nabla^{\mathrm{Sym}}\right|_{\operatorname{Elem}^{r} H^{\bullet}(\mathbb{P})}$ over the base $\wedge^{r} H^{\bullet}(\mathbb{P})$. More precisely, we have an embedding

$$
\chi_{2}: \operatorname{Elem}^{r} H^{\bullet}(\mathbb{P})_{\chi_{1}\left(\Delta\left(t^{\circ}\right)\right)} \hookrightarrow \wedge^{r} H^{\bullet}(\mathbb{P})_{\chi_{2}\left(\chi_{1}\left(\Delta\left(t^{\circ}\right)\right)\right)}
$$

and a pre-Saito structure $\nabla^{\wedge r}$ on the bundle

$$
\wedge^{r} H^{\bullet}(\mathbb{P}) \times\left(\wedge^{r} H^{\bullet}(\mathbb{P})_{\chi_{2}\left(\chi_{1}\left(\Delta\left(t^{\circ}\right)\right)\right)} \times \mathbb{P}^{1}\right) \rightarrow \wedge^{r} H^{\bullet}(\mathbb{P})_{\chi_{2}\left(\chi_{1}\left(\Delta\left(t^{\circ}\right)\right)\right)} \times \mathbb{P}^{1}
$$

which is a universal deformation of $\left.\nabla^{\mathrm{Sym}}\right|_{\operatorname{Elem}^{r} H^{\bullet}(\mathbb{P})}$ via the embedding $\chi_{2}$. The construction of $\nabla^{\wedge r}$ is given in [50, $\S 2.2$, p.235-236]. By the construction in the proof of [50, Corollary 1.7], the embedding $\chi_{2}$ is a primitive of the infinitesimal period mapping of the pre-Saito structure $\left.\nabla^{\text {Sym }}\right|_{\text {Elem }^{r} H^{\bullet}(\mathbb{P})}$ attached to $\sigma_{r-1} \wedge \sigma_{r-2} \wedge \cdots \wedge \sigma_{0}$, i.e.

$$
d \chi_{2}=\left.\left(z \nabla^{\mathrm{Sym}} \sigma_{r-2} \wedge \sigma_{r-1} \wedge \cdots \wedge \sigma_{0}\right)\right|_{\mathrm{Elem}^{r} H^{\bullet}(\mathbb{P}) \times\{z=0\}} .
$$

We normalize $\chi_{2}$ by the initial condition $\chi_{2}\left(\chi_{1}\left(\Delta\left(t^{\circ}\right)\right)\right)=t^{\circ} \sigma_{r-1} \wedge \sigma_{r-2} \wedge \cdots \wedge \sigma_{0}$. Since the restriction of $\nabla^{\text {Sym }}$ to the diagonal $\chi_{1} \circ \Delta: H^{2}(\mathbb{P}) \hookrightarrow \operatorname{Elem}^{r} H^{\bullet}(\mathbb{P})$ can be identified with the $r$-th wedge product of the small quantum connection of $\mathbb{P}$, we have

$$
\chi_{2}\left(\chi_{1}(\Delta(\tau))\right)=\tau \sigma_{r-1} \wedge \sigma_{r-2} \wedge \cdots \wedge \sigma_{0}, \quad \text { for } \tau \in H^{2}(\mathbb{P}) .
$$

The pre-Saito structure $\nabla^{\wedge r}$ together with a primitive section $c \sigma_{r-1} \wedge \cdots \wedge \sigma_{0}$ (for some $\left.c \in \mathbb{C}^{\times}\right)$and the metric $(\cdot, \cdot)_{\wedge \mathbb{P}}$ endows the base space $\wedge^{r} H^{\bullet}(\mathbb{P})$ with a Frobenius manifold structure [50, Corollary 2.10].

The main result of Ciocan-Fontanine-Kim-Sabbah [50, Theorem 2.13], [14, Theorem 4.1.1] implies that the germ of the pre-Saito structure $\nabla^{\wedge r}$ at $\chi_{2}\left(\chi_{1}\left(\Delta\left(t^{\circ}\right)\right)\right)$ with $t^{\circ}=(r-1) \pi i \sigma_{1}$ is isomorphic to the pre-Saito structure on the bundle

$$
H^{\bullet}(\mathbb{G}) \times\left(\left(H^{\bullet}(\mathbb{G}), 0\right) \times \mathbb{P}^{1}\right) \rightarrow\left(H^{\bullet}(\mathbb{G}), 0\right) \times \mathbb{P}^{1}
$$

defined by the big quantum connection of $\mathbb{G}$. By the construction in $[14, \S 3]$, the isomorphism between the above two pre-Saito bundles (6.3.2), (6.3.3) is induced by the Satake identification Sat between the fibers (up to a scalar multiple).

By the universal property (see [37, Definition 2.3], [50, §1.2]) of the pre-Saito structures $\nabla^{\otimes r}$ and $\nabla^{\wedge r}$, we obtain maps $\tilde{\chi}_{1}, \tilde{\chi}_{2}$ extending $\chi_{1}$ and $\chi_{2}$ which fit into the following commutative diagram.

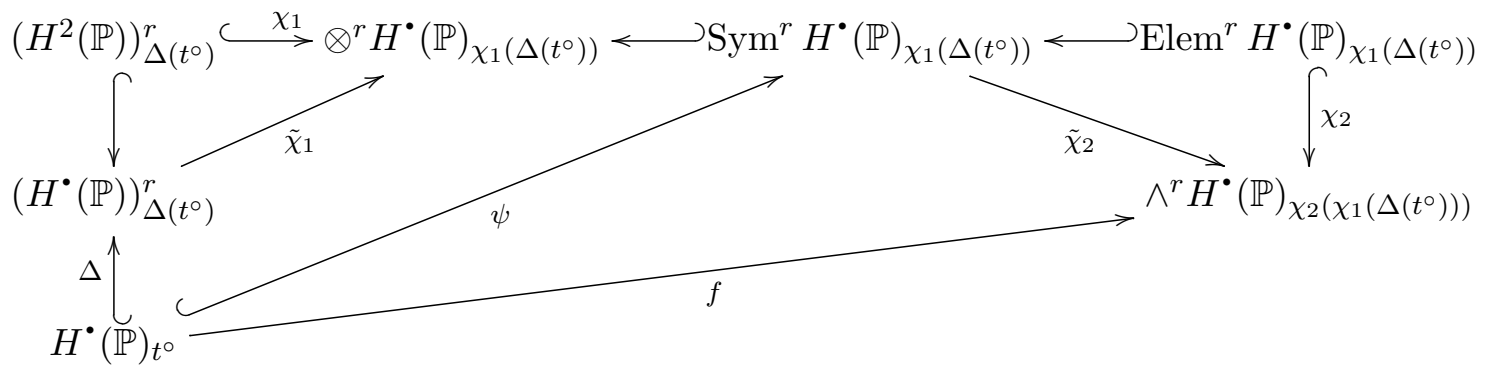

The maps $\tilde{\chi}_{1}, \tilde{\chi}_{2}$ are such that

- the pre-Saito structure over $\left(H^{\bullet}(\mathbb{P})\right)_{\Delta\left(t^{\circ}\right)}^{r}$ defined by the $r$-fold external tensor product of the big quantum connection of $\mathbb{P}$ equals the pull-back of $\nabla^{\otimes r}$ by $\tilde{\chi}_{1}$;

- the pre-Saito structure $\nabla^{\mathrm{Sym}}$ over $\operatorname{Sym}^{r} H^{\bullet}(\mathbb{P})_{\chi_{1}\left(\Delta\left(t^{\circ}\right)\right)}$ equals the pull-back of $\nabla^{\wedge r}$ by $\tilde{\chi}_{2}$. 
Again by the proof of [50, Corollary 1.7], the (non-injective) map $\tilde{\chi}_{1}$ is a primitive of the infinitesimal period mapping of the pre-Saito structure on $\left(H^{\bullet}(\mathbb{P})\right)_{\Delta\left(t^{\circ}\right)}^{r}$ (which is the $r$-fold external tensor product of the big quantum connection of $\mathbb{P}$ ) attached to $1 \otimes 1 \otimes \cdots \otimes 1$, and thus given by:

$$
\tilde{\chi}_{1}\left(\tau_{1}, \ldots, \tau_{r}\right)=\sum_{i=1}^{r} 1 \otimes \cdots \otimes \stackrel{i \text { th }}{\tau_{i}} \otimes \cdots \otimes 1 .
$$

Pre-composed with the diagonal map $\Delta: H^{\bullet}(\mathbb{P}) \rightarrow\left(H^{\bullet}(\mathbb{P})\right)^{r}, \tilde{\chi}_{1}$ induces a map $\psi: H^{\bullet}(\mathbb{P})_{t^{\circ}} \rightarrow$ $\operatorname{Sym}^{r} H^{\bullet}(\mathbb{P})_{\chi_{1}\left(\Delta\left(t^{\circ}\right)\right)}$. We have that the pre-Saito structure on $H^{\bullet}(\mathbb{P})_{t^{\circ}}$ defined as the $r$ fold tensor product of the big quantum connection of $\mathbb{P}$ is isomorphic to the pull-back of $\left.\nabla^{\otimes r}\right|_{\operatorname{Sym}^{r} H^{\bullet}(\mathbb{P})}$ by $\psi$, where the identification of fibers is the identity of $\otimes^{r} H^{\bullet}(\mathbb{P})$. Therefore the pull-back of the pre-Saito structure $\nabla^{\mathrm{Sym}}$ (defined as the anti-symmetric part of $\left.\left.\nabla^{\otimes r}\right|_{\operatorname{Sym}^{r} H^{\bullet}(\mathbb{P})}\right)$ by $\psi$ is naturally identified with the $r$-th wedge power $\nabla^{\wedge \mathbb{P}}$ of the big quantum connection of $\mathbb{P}$. Hence the composition $f=\tilde{\chi}_{2} \circ \psi$ pulls back the pre-Saito structure $\nabla^{\wedge r}$ to the pre-Saito structure $\nabla^{\wedge \mathbb{P}}$. Since the pre-Saito structure $\nabla^{\wedge r}$ is identified with the quantum connection $\nabla^{\mathbb{G}}$ of $\mathbb{G}$ near $f\left(t^{\circ}\right)$, we have $f^{*} \nabla^{\mathbb{G}} \cong \nabla^{\wedge \mathbb{P}}$ under the Satake identification. The scalar factor $\mathrm{i}^{r(r-1) / 2}$ is put to make the pairings match (see Lemma 6.2.8).

Finally we show that the map $f$ is an embedding of germs. It suffices to show that the differential of $f$ at the base point $t^{\circ}=(r-1) \pi i \sigma_{1}$ is injective. Since we already know that $\nabla^{\mathbb{G}}$ is pulled back to $\nabla^{\wedge \mathbb{P}}$, it suffices to check that $\left.z \nabla_{\sigma_{k}}^{\wedge \mathbb{P}}\left(\sigma_{r-1} \wedge \cdots \wedge \sigma_{0}\right)\right|_{t^{\circ}}, k=0, \ldots, N-1$ are linearly independent, as they correspond to $z \nabla_{d f\left(\sigma_{k}\right)}^{\mathbb{G}} 1=d f\left(\sigma_{k}\right)$. This follows from a straightforward computation.

Corollary 6.3.4. Let $\psi_{1}, \ldots, \psi_{N}$ be the idempotent basis of the quantum cohomology of $\mathbb{P}$ at $\mathbf{u} \in C_{N}(\mathbb{C})^{\sim}$ near $\mathbf{u}^{\circ}$ and write $\Delta_{i}=\left(\psi_{i}, \psi_{i}\right)_{\mathbb{P}}^{-1}$. Let $f$ be the embedding in Theorem 6.3.1. Then we have:

(1) the quantum product $\star_{\tau}$ of $\mathbb{G}$ is semisimple near $\tau=0$;

(2) the idempotent basis of $\mathbb{G}$ at $\tau=f(\mathbf{u})$ is given by

$$
\left(\prod_{a=1}^{r} \Delta_{i_{a}}\right) \operatorname{det}\left(\left(\psi_{i_{a}}, \sigma_{r-b}\right)_{\mathbb{P}}\right)_{1 \leqslant a, b \leqslant r} \operatorname{Sat}\left(\psi_{i_{1}} \wedge \cdots \wedge \psi_{i_{r}}\right)
$$

with $1 \leqslant i_{1}<i_{2}<\cdots<i_{r} \leqslant N$;

(3) the eigenvalues of the Euler multiplication $\left(E^{\mathbb{G}_{\star_{\tau}}}\right)$ of $\mathbb{G}$ at $\tau=f(\mathbf{u})$ are given by $u_{i_{1}}+\cdots+u_{i_{r}}$ with $1 \leqslant i_{1}<i_{2}<\cdots<i_{r} \leqslant N$.

Proof. The quantum product $\star_{\tau}$ for $\mathbb{G}$ is semisimple if and only if there exists a class $v \in H^{\bullet}(\mathbb{G})$ such that the endomorphism $\left(v \star_{\tau}\right)$ is semisimple with pairwise distinct eigenvalues. In this case, each eigenspace of $\left(v \star_{\tau}\right)$ contains a unique idempotent basis vector. On the other hand, since the quantum product of $\mathbb{P}$ is semisimple, we can find $w \in H^{*}(\mathbb{P})$ such that the action of $\left(w \star_{\mathbf{u}}\right)$ on $\wedge^{r} H^{\bullet}(\mathbb{P})$ is semisimple with pairwise distinct eigenvalues. Theorem 6.3.1 implies that $w \star_{\mathbf{u}}$ is conjugate to $d f_{\mathbf{u}}(w) \star_{f}(\mathbf{u})$ under Sat. This proves Part (1). Moreover the eigenspace $\mathbb{C} \operatorname{Sat}\left(\psi_{i_{1}} \wedge \cdots \wedge \psi_{i_{r}}\right), i_{1}<i_{2}<\cdots<i_{r}$ of $d f_{\mathbf{u}}(w) \star_{f(\mathbf{u})}$ contains a unique idempotent basis vector $\psi_{i_{1}, \ldots, i_{r}} \in H^{\bullet}(\mathbb{G})$. Set $\psi_{i_{1}, \ldots, i_{r}}=c \operatorname{Sat}\left(\psi_{i_{1}} \wedge \cdots \wedge \psi_{i_{r}}\right)$. Then we have

$$
\left(\psi_{i_{1}, \ldots, i_{r}}, \psi_{i_{1}, \ldots, i_{r}}\right)_{\mathbb{G}}=\left(\psi_{i_{1}, \ldots, i_{r}} \star_{f(\mathbf{u})} \psi_{i_{1}, \ldots, i_{r}}, 1\right)_{\mathbb{G}}=\left(\psi_{i_{1}, \ldots, i_{r}}, 1\right)_{\mathbb{G}} .
$$

This implies by Lemma 6.2.8 that:

$$
c^{2}\left(\psi_{i_{1}} \wedge \cdots \wedge \psi_{i_{r}}, \psi_{i_{1}} \wedge \cdots \wedge \psi_{i_{r}}\right)_{\wedge \mathbb{P}}=c\left(\psi_{i_{1}} \wedge \cdots \wedge \psi_{i_{r}}, \sigma_{r-1} \wedge \cdots \wedge \sigma_{0}\right)_{\wedge \mathbb{P}} .
$$

Part (2) follows from this. Part (3) follows from the fact that the Euler multiplication $\left(E^{\mathbb{P}} \star_{\tau}\right)$ on $\wedge^{r} H^{\bullet}(\mathbb{P})$ is conjugate to $\left(E^{\mathbb{G}} \star_{f(\mathbf{u})}\right)$ on $H^{\bullet}(\mathbb{G})$ by Sat. 
6.4. The wedge product of MRS. Let $M=\left(V,[\cdot, \cdot),\left\{v_{1}, \ldots, v_{N}\right\}, m: v_{i} \mapsto u_{i}, e^{i \phi}\right)$ be an MRS (see $\S 4.2$ ). The $r$-th wedge product $\wedge^{r} M$ is defined by the data:

- the vector space $\wedge^{r} V$;

- the pairing $\left[\alpha_{1} \wedge \cdots \wedge \alpha_{r}, \beta_{1} \wedge \cdots \wedge \beta_{r}\right):=\operatorname{det}\left(\left[\alpha_{i}, \beta_{j}\right)\right)_{1 \leqslant i, j \leqslant r}$

- the basis $\left\{v_{i_{1}} \wedge \cdots \wedge v_{i_{r}}: 1 \leqslant i_{1}<\cdots<i_{r} \leqslant N\right\}$;

- the marking $m: v_{i_{1}} \wedge \cdots \wedge v_{i_{r}} \mapsto u_{i_{1}}+\cdots+u_{i_{r}}$;

- the same phase $e^{\mathrm{i} \phi}$.

Note that the basis $\left\{v_{i_{1}} \wedge \cdots \wedge v_{i_{r}}\right\}$ is determined up to sign because $\left\{v_{1}, \ldots, v_{N}\right\}$ is an unordered basis. In other words, $\wedge^{r} M$ is defined up to sign. The following lemma shows that the above data is indeed an MRS. Recall that $h_{\phi}(u)=\operatorname{Im}\left(e^{-i \phi} u\right)$.

Lemma 6.4.1. Let $1 \leqslant i_{1}<\cdots<i_{r} \leqslant N, 1 \leqslant j_{1}<\cdots<j_{r} \leqslant N$ be increasing sequences of integers. If $h_{\phi}\left(u_{i_{1}}+\cdots+u_{i_{r}}\right)=h_{\phi}\left(u_{j_{1}}+\cdots+u_{j_{r}}\right)$, we have

$$
\left[v_{i_{1}} \wedge \cdots \wedge v_{i_{r}}, v_{j_{1}} \wedge \cdots \wedge v_{j_{r}}\right)= \begin{cases}1 & \text { if } i_{a}=j_{a} \text { for all } a=1, \ldots, r ; \\ 0 & \text { otherwise. }\end{cases}
$$

Proof. Suppose that $\prod_{a=1}^{r}\left[v_{i_{a}}, v_{j_{\sigma(a)}}\right) \neq 0$ for some permutation $\sigma \in \mathfrak{S}_{r}$. Then for each $a$, we have either $i_{a}=j_{\sigma(a)}$ or $h_{\phi}\left(u_{i_{a}}\right)>h_{\phi}\left(u_{j_{\sigma(a)}}\right)$ by (4.2.1). The assumption implies that $i_{a}=j_{\sigma(a)}$ for all $a$; this happens only when $\sigma=$ id. The lemma follows.

The wedge product of an admissible MRS is not necessarily admissible.

Remark 6.4.2. We can define the tensor product of two MRSs similarly.

Remark 6.4.3. We can show that, when two MRSs $M_{1}$ and $M_{2}$ are related by mutations (see $\S 4.3), \wedge^{r} M_{1}$ and $\wedge^{r} M_{2}$ are also related by mutations. We omit a proof of this fact since we do not use it in this paper; the details are left to the reader.

6.5. MRS of Grassmannian. Using the result from $\S 6.3$, we show that the MRS of $\mathbb{G}$ is isomorphic to the wedge product of the MRS of $\mathbb{P}$. By the results in $\S 6.3$, the flat connections $\nabla^{\mathbb{P}}$ and $\nabla^{\wedge \mathbb{P}}$ on the base $C_{N}(\mathbb{C})^{\sim}$ give isomonodromic deformations of the quantum connections of $\mathbb{P}$ and $\mathbb{G}$ respectively. Therefore we can define the MRSs of $\mathbb{P}$ or $\mathbb{G}$ at a point $\mathbf{u} \in C_{N}(\mathbb{C})^{\sim}$ with respect to an admissible phase $\phi$ following $\S 2.7$ (and $\S 4.5$ ).

Proposition 6.5.1. Let $\left(H^{\bullet}(\mathbb{P}),[\cdot, \cdot),\left\{A_{1}, \ldots, A_{N}\right\}, A_{i} \mapsto u_{i}, e^{\mathrm{i} \phi}\right)$ be the MRS of $\mathbb{P}$ at $\mathbf{u} \in$ $C_{N}(\mathbb{C})^{\sim}$ with respect to phase $\phi$. Suppose that the phase $\phi$ is admissible for the $r$-th wedge $\left\{u_{i_{1}}+\cdots+u_{i_{r}}: i_{1}<i_{2}<\cdots<i_{r}\right\}$ of the spectrum of $\left(E \star_{\mathbf{u}}\right)$. Then the MRS of $\mathbb{G}$ at $\mathbf{u}$ with respect to $\phi$ is given by the asymptotic basis

$$
\frac{1}{(2 \pi \mathbf{i})^{r(r-1) / 2}} e^{-(r-1) \pi \mathbf{i} \sigma_{1}} \operatorname{Sat}\left(A_{i_{1}} \wedge \cdots \wedge A_{i_{r}}\right)
$$

marked by $u_{i_{1}}+\cdots+u_{i_{r}}$ with $1 \leqslant i_{1}<i_{2}<\cdots<i_{r} \leqslant N$, where Sat is the Satake identification (6.2.4). In other words, one has $\mathfrak{R e f}(\mathbb{G}, \mathbf{u}, \phi) \cong \wedge^{r} \mathfrak{R e f}(\mathbb{P}, \mathbf{u}, \phi)$ (see $\left.\S 6.4\right)$.

Proof. Let $y_{1}(z), \ldots, y_{N}(z)$ be the basis of asymptotically exponential flat sections for $\left.\nabla^{\mathbb{P}}\right|_{\mathbf{u}}$ corresponding to $A_{1}, \ldots, A_{N}$. They are characterized by the asymptotic condition $y_{i}(z) \sim$ $e^{-u_{i} / z}\left(\Psi_{i}+O(z)\right)$ in the sector $|\arg z-\phi|<\frac{\pi}{2}+\epsilon$ for some $\epsilon>0$, where $\Psi_{1}, \ldots, \Psi_{N}$ are normalized idempotent basis for $\mathbb{P}$. For $1 \leqslant i_{1}<i_{2}<\cdots<i_{r} \leqslant N, y_{i_{1}, \ldots, i_{r}}(z):=$ $y_{i_{1}}(z) \wedge \cdots \wedge y_{i_{r}}(z)$ gives a flat section for $\left.\nabla^{\wedge \mathbb{P}}\right|_{\mathbf{u}}$ and satisfies the asymptotic condition

$$
y_{i_{1}, \ldots, i_{r}}(z) \sim e^{-\left(u_{i_{1}}+\cdots+u_{i_{r}}\right) / z}\left(\Psi_{i_{1}} \wedge \cdots \wedge \Psi_{i_{r}}+O(z)\right)
$$


in the same sector. Note that $i^{r(r-1) / 2} \operatorname{Sat}\left(\Psi_{i_{1}} \wedge \cdots \wedge \Psi_{i_{r}}\right)$ give (analytic continuation of) the normalized idempotent basis for $\mathbb{G}$ by Theorem 6.3.1 and Corollary 6.3.4. Therefore the asymptotically exponential flat sections $\dot{i}^{r(r-1) / 2} \operatorname{Sat}\left(y_{i_{1}, \ldots, i_{r}}(z)\right)$ give rise to the asymptotic basis of $\mathbb{G}$ for $\mathbf{u}$ and $\phi$.

Let $S^{\mathbb{P}}(\tau, z) z^{-\mu^{\mathbb{P}}} z^{\rho^{\mathbb{P}}}$ with $\tau \in H^{\bullet}(\mathbb{P})$ denote the fundamental solution for the big quantum connection of $\mathbb{P}$ as in Remark 2.3.2. The group elements $S^{\mathbb{P}}(\tau, z), z^{-\mu^{\mathbb{P}}}, z^{\rho^{\mathbb{P}}} \in G L\left(H^{\bullet}(\mathbb{P})\right)$ naturally act on the $r$-th wedge representation $\wedge^{r} H^{\bullet}(\mathbb{P})$; we denote these actions by the same symbols. Define the $\operatorname{End}\left(H^{\bullet}(\mathbb{G})\right)$-valued function $S^{\mathbb{G}}(z)$ by

$$
S^{\mathbb{G}}(z) \operatorname{Sat}(\alpha)=e^{\left(t^{\circ} \cup\right) / z} \operatorname{Sat}\left(S^{\mathbb{P}}\left(t^{\circ}, z\right) \alpha\right) \quad \text { with } t^{\circ}:=(r-1) \pi i \sigma_{1}
$$

for all $\alpha \in \wedge^{r} H^{\bullet}(\mathbb{P})$. This satisfies $S^{\mathbb{G}}(z=\infty)=\mathrm{id}_{H^{\bullet}(\mathbb{G})}$. Using Lemma 6.2 .7 and the 'classical' Satake for the cup product by $c_{1}$ (cf. Proposition 6.2.5), we find:

$$
z^{\rho^{\mathbb{G}}} \operatorname{Sat}(\alpha)=\operatorname{Sat}\left(z^{\rho^{\mathbb{P}}} \alpha\right), \quad z^{\mu^{\mathbb{G}}} \operatorname{Sat}(\alpha)=\operatorname{Sat}\left(z^{\mu^{\mathbb{P}}} \alpha\right)
$$

where $\mu^{\mathbb{G}}$ is the grading operator of $\mathbb{G}$ and $\rho^{\mathbb{G}}=\left(c_{1}(\mathbb{G}) \cup\right)$. Therefore:

$$
S^{\mathbb{G}}(z) z^{-\mu^{\mathbb{G}}} z^{\rho^{\mathbb{G}}} \operatorname{Sat}(\alpha)=e^{\left(t^{\circ} \cup\right) / z} \operatorname{Sat}\left(S^{\mathbb{P}}\left(t^{\circ}, z\right) z^{-\mu^{\mathbb{P}}} z^{\rho^{\mathbb{P}}} \alpha\right) .
$$

These sections are flat for $\left.\nabla^{\mathbb{G}}\right|_{\tau=0}$ by the 'quantum' Satake (or Theorem 6.3.1). Note that we have:

$$
z^{\mu^{\mathbb{G}}} S^{\mathbb{G}}(z) z^{-\mu^{\mathbb{G}}} \operatorname{Sat}(\alpha)=e^{\left(t^{\circ} \cup\right)} \operatorname{Sat}\left(z^{\mu^{\mathbb{P}}} S^{\mathbb{P}}\left(t^{\circ}, z\right) z^{-\mu^{\mathbb{P}}} \alpha\right)
$$

By Lemma 6.5 .3 below, we have $\left[z^{\mu^{\mathbb{G}}} S^{\mathbb{G}}(z) z^{-\mu^{\mathbb{G}}}\right]_{z=\infty}=\operatorname{id}_{H} \cdot(\mathbb{G})$. Hence $S^{\mathbb{G}}(z) z^{-\mu^{\mathbb{G}}} z^{\rho^{\mathbb{G}}}$ coincides with the fundamental solution of $\mathbb{G}$ from Proposition 2.3.1.

The asymptotic basis $A_{1}, \ldots, A_{N}$ of $\mathbb{P}$ are related to $y_{1}(z), \ldots, y_{N}(z)$ as (see $\left.\S 4.5\right)$

$$
\left.y_{i}(z)\right|_{\substack{\text { parallel transport } \\ \text { to } \mathbf{u}^{\circ}=\left\{\tau_{\mathbb{P}}=t^{\circ}\right\}}}=\frac{1}{(2 \pi)^{\operatorname{dim} \mathbb{P} / 2}} S^{\mathbb{P}}\left(t^{\circ}, z\right) z^{-\mu^{\mathbb{P}}} z^{\rho^{\mathbb{P}}} A_{i} .
$$

This together with (6.5.2) and the definition of $y_{i_{1}, \ldots, i_{r}}(z)$ implies that

$$
\begin{aligned}
& \left.\dot{i}^{r(r-1) / 2} \operatorname{Sat}\left(y_{i_{1}, \ldots, i_{r}}(z)\right)\right|_{\text {parallel transport to } \mathbf{u}^{\circ}} \\
& \quad=\frac{1}{(2 \pi)^{\operatorname{dim} \mathbb{G} / 2}} S^{\mathbb{G}}(z) z^{-\mu^{\mathbb{G}}} z^{\rho^{\mathbb{G}}}\left[\frac{1}{(-2 \pi \mathbf{i})^{r(r-1) / 2}} e^{-\left(t^{\circ} \cup\right)} \operatorname{Sat}\left(A_{i_{1}} \wedge \cdots \wedge A_{i_{r}}\right)\right] .
\end{aligned}
$$

Recall that the base point $\mathbf{u}^{\circ} \in C_{N}(\mathbb{C})^{\sim}$ corresponds to $0 \in H^{\bullet}(\mathbb{G})$ for $\mathbb{G}$ and to $t^{\circ} \in H^{\bullet}(\mathbb{P})$ for $\mathbb{P}$. The conclusion follows from this. (Note also that the asymptotic basis is defined only up to sign.)

Lemma 6.5.3. The fundamental solution $S(\tau, z) z^{-\mu} z^{\rho}$ in Remark 2.3.2 satisfies $\left[z^{\mu} S(\tau, z) z^{-\mu}\right]_{z=\infty}=e^{-(\tau \cup)}$ for $\tau \in H^{2}(F)$.

Proof. The differential equation for $T(\tau, z)=z^{\mu} S(\tau, z) z^{-\mu}$ in the $\tau$-direction reads $\partial_{\alpha} T(\tau, z)+$ $z^{-1}\left(z^{\mu}\left(\alpha \star_{\tau}\right) z^{-\mu}\right) T(\tau, z)=0$ for $\alpha \in H^{\bullet}(F)$. If $\tau, \alpha \in H^{2}(F)$, we have that $z^{-1}\left(z^{\mu}\left(\alpha \star_{\tau}\right) z^{-\mu}\right)$ is regular at $z=\infty$ and equals $(\alpha \cup)$ there. Here we use the fact that $F$ is Fano and the divisor axiom (see Remark 2.1.2). The conclusion follows by solving the differential equation along $H^{2}(F)$.

Remark 6.5.4. When we identify the MRS of $\mathbb{G}$ with the $r$-th wedge of the MRS of $\mathbb{P}$, we should use the identification $(2 \pi i)^{-r(r-1) / 2} e^{-(r-1) \pi \mathrm{i} \sigma_{1}}$ Sat: $\wedge^{r} H^{\bullet}(\mathbb{P}) \cong H^{\bullet}(\mathbb{G})$ that respects the pairing $[\cdot, \cdot)$ in $(3.4 .5)$. 
6.6. The wedge product of Gamma basis. We show that the $r$-th wedge of the Gamma basis $\left\{\widehat{\Gamma}_{\mathbb{P}} \mathrm{Ch}(\mathcal{O}(i))\right\}$ given by Beilinson's exceptional collection for $\mathbb{P}$ matches up with the Gamma basis $\left\{\widehat{\Gamma}_{\mathbb{G}} \mathrm{Ch}\left(S^{\nu} V^{*}\right)\right\}$ given by Kapranov's exceptional collection for $\mathbb{G}$. In view of the truth of Gamma Conjecture II for $\mathbb{P}$ (Theorem 5.0.1) and Proposition 6.5.1, the following proposition completes the proof of Gamma Conjecture II for $\mathbb{G}$.

Proposition 6.6.1. Let $N-r \geqslant \nu_{1} \geqslant \nu_{2} \geqslant \cdots \geqslant \nu_{r} \geqslant 0$ be a partition in an $r \times(N-r)$ rectangle. We have

$$
\widehat{\Gamma}_{\mathbb{G}} \operatorname{Ch}\left(S^{\nu} V^{*}\right)=(2 \pi \mathbf{i})^{-\left(\begin{array}{c}
r \\
2
\end{array}\right)} e^{-(r-1) \pi \mathbf{i} \sigma_{1}} \operatorname{Sat}\left(\widehat{\Gamma}_{\mathbb{P}} \operatorname{Ch}\left(\mathcal{O}\left(\nu_{1}+r-1\right)\right) \wedge \cdots \wedge \widehat{\Gamma}_{\mathbb{P}} \operatorname{Ch}\left(\mathcal{O}\left(\nu_{r}\right)\right)\right) .
$$

We give an elementary algebraic proof of Proposition 6.6.1 in this section. A geometric proof will be discussed in $\S 6.8$. Let $x_{1}, \ldots, x_{r}$ denote the Chern roots of $V^{*}$ as before. (Recall that $V$ is the tautological bundle on $\mathbb{G}$.) Let $h=c_{1}(\mathcal{O}(1))$ denote the hyperplane class on $\mathbb{P}=\mathbb{P}^{N-1}$.

Lemma 6.6.2. Let $f_{1}(z), \ldots, f_{r}(z)$ be power series in $\mathbb{C} \llbracket z \rrbracket$. One has

$$
\operatorname{Sat}\left(f_{1}(h) \wedge f_{2}(h) \wedge \cdots \wedge f_{r}(h)\right)=\frac{\operatorname{det}\left(f_{j}\left(x_{i}\right)\right)_{1 \leqslant i, j \leqslant r}}{\prod_{i<j}\left(x_{i}-x_{j}\right)} .
$$

Proof. For monomial $f_{i}(z)$ 's this follows by the definition of the Schur polynomials (6.2.1) and the Satake identification Sat (6.2.4). The general case follows by linearity.

Lemma 6.6.3. One has

$$
\operatorname{Ch}\left(S^{\nu} V^{*}\right)=\frac{\operatorname{det}\left(e^{2 \pi \mathrm{i} x_{i}\left(\nu_{j}+r-j\right)}\right)_{1 \leqslant i, j \leqslant r}}{\prod_{i<j}\left(e^{2 \pi \mathrm{i} x_{i}}-e^{2 \pi \mathrm{i} x_{j}}\right)}
$$

Proof. Let $L_{1}, \ldots, L_{r}$ be the $K$-theoretic Chern roots of $V^{*}$ so that $\left[V^{*}\right]=L_{1}+\cdots+L_{r}$. The $K$-class $\left[S^{\nu} V^{*}\right]$ can be expressed as the Schur polynomial $\sigma_{\nu}\left(L_{1}, \ldots, L_{r}\right)$ in $L_{1}, \ldots, L_{r}$. The lemma follows from the definition of the Schur polynomial (6.2.1) and $\operatorname{Ch}\left(L_{i}\right)=e^{2 \pi \mathrm{i} x_{i}}$.

Lemma 6.6.4. The Gamma class of $\mathbb{G}$ is given by

$$
\widehat{\Gamma}_{\mathbb{G}}=(2 \pi \mathbf{i})^{-\left(\begin{array}{c}
r \\
2
\end{array}\right) e^{-(r-1) \pi \mathrm{i} \sigma_{1}}} \prod_{i<j} \frac{e^{2 \pi \mathrm{i} x_{i}}-e^{2 \pi \mathbf{i} x_{j}}}{x_{i}-x_{j}} \prod_{i=1}^{r} \Gamma\left(1+x_{i}\right)^{N}
$$

Proof. The tangent bundle $T \mathbb{G}$ of $\mathbb{G}$ is isomorphic to $\operatorname{Hom}(V, Q)$, where $Q$ is the universal quotient bundle. The exact sequence $0 \rightarrow V \rightarrow \mathcal{O}_{\mathbb{G}}^{\oplus N} \rightarrow Q \rightarrow 0$ implies that $[T \mathbb{G}]=$ $\left[\operatorname{Hom}(V, \mathcal{O})^{\oplus N}\right]-[\operatorname{Hom}(V, V)]=N\left[V^{*}\right]-\left[V^{*} \otimes V\right]$ in the $K$-group. Thus we have:

$$
\widehat{\Gamma}_{\mathbb{G}}=\frac{\prod_{i=1}^{r} \Gamma\left(1+x_{i}\right)^{N}}{\prod_{1 \leqslant i, j \leqslant r} \Gamma\left(1+x_{i}-x_{j}\right)}
$$

The denominator of the right-hand side equals

$$
\begin{aligned}
& \prod_{i<j}\left(\Gamma\left(1+x_{i}-x_{j}\right) \Gamma\left(1-x_{i}+x_{j}\right)\right)=\prod_{i<j} \frac{2 \pi \mathrm{i}\left(x_{i}-x_{j}\right)}{e^{\pi \mathrm{i}\left(x_{i}-x_{j}\right)}-e^{-\pi \mathrm{i}\left(x_{i}-x_{j}\right)}} \\
& =(2 \pi \mathrm{i})^{\left(\begin{array}{c}
r \\
2
\end{array}\right)} \prod_{i<j}\left(x_{i}-x_{j}\right) \frac{e^{(r-1) \pi \mathrm{i} \sigma_{1}}}{\prod_{i<j}\left(e^{2 \pi \mathrm{i} x_{i}}-e^{2 \pi \mathrm{i} x_{j}}\right)}
\end{aligned}
$$

where we used the Gamma function identity (3.4.7) and $e^{\pi \mathrm{i}\left(x_{i}-x_{j}\right)}-e^{-\pi \mathrm{i}\left(x_{i}-x_{j}\right)}=\left(e^{2 \pi \mathrm{i} x_{i}}-\right.$ $\left.e^{2 \pi \mathbf{i} x_{j}}\right) e^{-\pi \mathbf{i}\left(x_{i}+x_{j}\right)}$. The Lemma follows. 
Proof of Proposition 6.6.1. By Lemmata 6.6.3 and 6.6.4, we have

$$
\widehat{\Gamma}_{\mathbb{G}} \operatorname{Ch}\left(S^{\nu} V^{*}\right)=(2 \pi \mathbf{i})^{-\left(\begin{array}{c}
r \\
2
\end{array}\right)} e^{-(r-1) \pi \mathbf{i} \sigma_{1}} \frac{\left.\operatorname{det}\left(e^{2 \pi \mathbf{i} x_{i}\left(\nu_{j}+r-j\right)}\right)\right)_{1 \leqslant i, j \leqslant r}}{\prod_{i<j}\left(x_{i}-x_{j}\right)} \prod_{i=1}^{r} \Gamma\left(1+x_{i}\right)^{N} .
$$

By Lemma 6.6.2, we have

$$
\operatorname{Sat}\left(\widehat{\Gamma}_{\mathbb{P}} \operatorname{Ch}\left(\mathcal{O}\left(\nu_{1}+r-1\right)\right) \wedge \cdots \wedge \widehat{\Gamma}_{\mathbb{P}} \operatorname{Ch}\left(\mathcal{O}\left(\nu_{r}\right)\right)\right)=\frac{\operatorname{det}\left(\Gamma\left(1+x_{i}\right)^{N} e^{2 \pi \mathrm{i} x_{i}\left(\nu_{j}+r-j\right)}\right)_{1 \leqslant i, j \leqslant r}}{\prod_{i<j}\left(x_{i}-x_{j}\right)} .
$$

The conclusion follows from these formulas.

We have now completed the proof of Gamma Conjecture II for $\mathbb{G}$.

6.7. Gamma Conjecture I for Grassmannians. Here we prove that $\mathbb{G}$ satisfies Gamma Conjecture I. We may assume that $r \leqslant N / 2$ by replacing $r$ with $N-r$ if necessary. Recall that $\mathbb{G}$ satisfies Property $\mathcal{O}$ (Remark 6.2.9) and that the quantum product $\star_{\tau}$ of $\mathbb{G}$ is semisimple near $\tau=0$ (Corollary 6.3.4). Let $\phi$ be an admissible phase for the spectrum of $\left(c_{1}(\mathbb{G}) \star_{0}\right)$, sufficiently close to zero. Let $T_{\mathbb{G}}=N \sin (\pi r / N) / \sin (\pi / N)$ denote the biggest eigenvalue of $\left(c_{1}(\mathbb{G}) \star_{0}\right)$. In view of Proposition 4.6.7, it suffices to show that the member $A_{\mathbb{G}}$ of the asymptotic basis (at $\tau=0$ with respect to $\phi$ ) corresponding to $T_{\mathbb{G}}$ is $\pm \widehat{\Gamma}_{\mathbb{G}}$. By Proposition 6.5.1, we have

$$
A_{\mathbb{G}}=(2 \pi \mathrm{i})^{-\left(\begin{array}{l}
r \\
2
\end{array}\right)} e^{-(r-1) \pi \mathrm{i} \sigma_{1}} \operatorname{Sat}\left(A_{0} \wedge \cdots \wedge A_{r-1}\right)
$$

where $A_{k} \in H^{\bullet}(\mathbb{P})$ is the member of the asymptotic basis for $\mathbb{P}$ at $\tau=(r-1) \pi i \sigma_{1}$ with respect to $\phi$, corresponding to the eigenvalue $N e^{\pi \mathrm{i}(r-1) / N} e^{-2 \pi \mathrm{i} k / N}$. By the discussion in $\S 5$, we know that $A_{k}=\widehat{\Gamma}_{\mathbb{P}} \operatorname{Ch}(\mathcal{O}(k))$. Here we use the condition that $\phi$ is close to zero and $r \leqslant N / 2$. Thus Proposition 6.6.1 implies the equality $A_{\mathbb{G}}= \pm \widehat{\Gamma}_{\mathbb{G}}$. Gamma Conjecture I for $\mathbb{G}$ is proved.

The proof of Theorem 6.1.1 is now complete.

6.8. The abelian/non-abelian correspondence and the Gamma basis. We give an alternative proof of Proposition 6.6.1 in the spirit of the abelian/non-abelian correspondence $[9,14]$. The product $\mathbb{P}^{\times r}=\mathbb{P}^{N-1} \times \cdots \times \mathbb{P}^{N-1}(r$ factors $)$ of projective spaces and the Grassmannian $\mathbb{G}=G(r, N)$ arise respectively as the GIT quotients $\operatorname{Hom}\left(\mathbb{C}^{r}, \mathbb{C}^{N}\right) / /\left(\mathbb{C}^{\times}\right)^{r}$ and $\operatorname{Hom}\left(\mathbb{C}^{r}, \mathbb{C}^{N}\right) / / G L(r, \mathbb{C})$ of the same vector space $\operatorname{Hom}\left(\mathbb{C}^{r}, \mathbb{C}^{N}\right)$. We relate them by the following diagram:

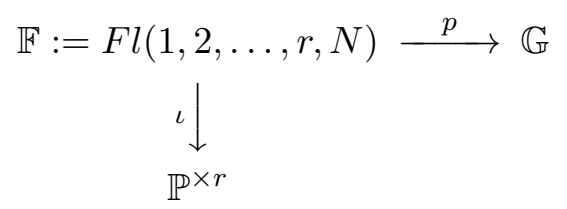

where $\mathbb{F}$ is the partial flag variety parameterizing flags $0=V_{0} \subset V_{1} \subset V_{2} \subset \cdots \subset V_{r} \subset \mathbb{C}^{N}$ with $\operatorname{dim} V_{i}=i, p$ is the projection forgetting the intermediate flags $V_{1}, \ldots, V_{r-1}$ and $\iota$ is the real-analytic inclusion sending a flag $\left\{V_{1} \subset \cdots \subset V_{r}\right\}$ to a collection $\left(V_{1}, V_{2} \ominus V_{1}, \ldots, V_{r} \ominus V_{r-1}\right)$ of lines, where $V_{i} \ominus V_{i-1}$ is the orthogonal complement of $V_{i-1}$ in $V_{i}$. The projection $p$ is a fiber bundle with fiber $F l(1,2, \ldots, r)$. The normal bundle $\mathcal{N}_{\iota}$ of the inclusion $\iota$ is isomorphic to the conjugate $\overline{T_{p}}$ of the relative tangent bundle $T_{p}$ of $p$, as a topological vector bundle. 
Let $L_{i}=\left(V_{i} / V_{i-1}\right)^{*}$ be the line bundle on $\mathbb{F}$ and set $x_{i}:=c_{1}\left(L_{i}\right)$. We have $\operatorname{Euler}\left(T_{p}\right)=$ $\prod_{i<j}\left(x_{i}-x_{j}\right)=(-1)^{\left(\begin{array}{c}r \\ 2\end{array}\right)} \operatorname{Euler}\left(\mathcal{N}_{\iota}\right)$ and:

$$
\begin{aligned}
H^{\bullet}(\mathbb{G}) & \cong \mathbb{C}\left[x_{1}, \ldots, x_{r}\right]^{\mathfrak{S}_{r}} /\left\langle h_{N-r+1}, \ldots, h_{N}\right\rangle ; \\
H^{\bullet}(\mathbb{F}) & \cong \mathbb{C}\left[x_{1}, \ldots, x_{r}\right] /\left\langle h_{N-r+1}, \ldots, h_{N}\right\rangle \\
H^{\bullet}\left(\mathbb{P}^{\times r}\right) & \cong \mathbb{C}\left[x_{1}, \ldots, x_{r}\right] /\left\langle x_{1}^{N}, \ldots, x_{r}^{N}\right\rangle .
\end{aligned}
$$

The injective map

$$
\iota_{*} p^{*}: H^{\bullet}(\mathbb{G}) \longrightarrow H^{\bullet}\left(\mathbb{P}^{\times r}\right) \cong \otimes^{r} H^{\bullet}(\mathbb{P})
$$

identifies $H^{\bullet}(\mathbb{G})$ with the anti-symmetric part $\wedge^{r} H^{\bullet}(\mathbb{P}) \subset \otimes^{r} H^{\bullet}(\mathbb{P})$, where we embed $\wedge^{r} H^{\bullet}(\mathbb{P})$ into $\otimes^{r} H^{\bullet}(\mathbb{P})$ by $\alpha_{1} \wedge \cdots \wedge \alpha_{r} \mapsto \sum_{\sigma \in \mathfrak{S}_{r}} \operatorname{sgn}(\sigma) \alpha_{\sigma(1)} \otimes \cdots \otimes \alpha_{\sigma(r)}$. This is inverse to the Satake

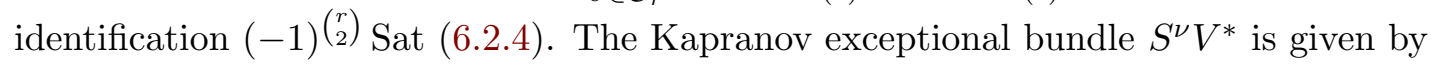

$$
S^{\nu} V^{*}=p_{*}\left(L^{\nu}\right)
$$

with $L^{\nu}=L_{1}^{\otimes \nu_{1}} \otimes \cdots \otimes L_{r}^{\otimes \nu_{r}}$. Because $\iota^{*} T\left(\mathbb{P}^{\times r}\right) \cong p^{*} T \mathbb{G} \oplus T_{p} \oplus \overline{T_{p}}$ as topological bundles, we find

$$
\iota^{*} \widehat{\Gamma}_{\mathbb{P}^{\times r}}=p^{*} \widehat{\Gamma}_{\mathbb{G}} \cup \widehat{\Gamma}\left(T_{p}\right) \cup \widehat{\Gamma}\left(\overline{T_{p}}\right)=p^{*} \widehat{\Gamma}_{\mathbb{G}} \cup \operatorname{Td}\left(T_{p}\right) e^{-\pi \mathrm{i} c_{1}\left(T_{p}\right)} .
$$

By the Grothendieck-Riemann-Roch theorem, we have

$$
\operatorname{Ch}\left(S^{\nu} V^{*}\right)=\operatorname{Ch}\left(p_{*}\left(L^{\nu}\right)\right)=(2 \pi i)^{-\left(\begin{array}{c}
r \\
2
\end{array}\right)} p_{*}\left(\operatorname{Ch}\left(L^{\nu}\right) \operatorname{Td}\left(T_{p}\right)\right) .
$$

Combining (6.8.1) and (6.8.2), we have:

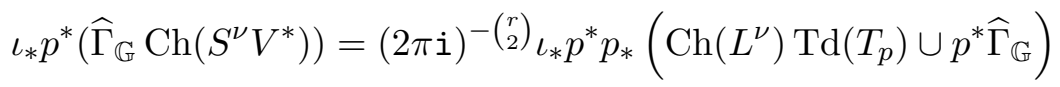

$$
\begin{aligned}
& =(2 \pi \mathbf{i})^{-\left(\begin{array}{c}
r \\
2
\end{array}\right)} \iota_{*} p^{*} p_{*}\left(\mathrm{Ch}\left(L^{\nu}\right) e^{\pi \mathrm{i} c_{1}\left(T_{p}\right)} \iota^{*} \widehat{\Gamma}_{\mathbb{P} \times r}\right) \\
& =(2 \pi \mathrm{i})^{-\left(\begin{array}{c}
r \\
2
\end{array}\right)} \iota_{*} p^{*} p_{*} \iota^{*}\left(\mathrm{Ch}\left(\mathcal{O}\left(\nu_{1}+r-1\right) \otimes \cdots \otimes \mathcal{O}\left(\nu_{r}\right)\right) e^{-(r-1) \pi \mathrm{i} \sigma_{1}} \widehat{\Gamma}_{\mathbb{P} \times r}\right) .
\end{aligned}
$$

In the last line we used $\pi \mathbf{i} c_{1}\left(T_{p}\right)=\pi \mathbf{i}\left((r-1) x_{1}+(r-3) x_{2}+\cdots-(r-1) x_{r}\right)=-(r-$ 1) $\pi \mathrm{i} \sigma_{1}+2 \pi \mathrm{i}\left((r-1) x_{1}+(r-2) x_{2}+\cdots+x_{r-1}\right)$. The map $\iota_{*} p^{*} p_{*} \iota^{*}: \otimes^{r} H^{\bullet}(\mathbb{P}) \rightarrow \otimes^{r} H^{\bullet}(\mathbb{P})$ is the anti-symmetrization map and the quantity in the last line can be identified an element

$$
(-2 \pi \mathbf{i})^{-\left(\begin{array}{l}
r \\
2
\end{array}\right)} e^{-(r-1) \pi \mathbf{i} \sigma_{1}}\left(\widehat{\Gamma}_{\mathbb{P}} \operatorname{Ch}\left(\mathcal{O}\left(\nu_{1}+r-1\right)\right) \wedge \cdots \wedge \widehat{\Gamma}_{\mathbb{P}} \operatorname{Ch}\left(\mathcal{O}\left(\nu_{r}\right)\right)\right) .
$$

of the wedge product $\wedge^{r} H^{\bullet}(\mathbb{P})$. Since $\iota_{*} p^{*}$ is inverse to $(-1)^{\left(\begin{array}{c}r \\ 2\end{array}\right)}$ Sat, the conclusion of Proposition 6.6.1 follows.

Remark 6.8.3. Most of the above discussions can be applied to a general abelian/non-abelian correspondence. The bundle $T_{p}$ corresponds to the sum of positive roots.

\section{Appendix A. $\zeta$-FunCtion REgularization}

The $\zeta$-function regularization (1.1.1) of the $S^{1}$-equivariant Euler class $e_{S^{1}}\left(\mathcal{N}_{+}\right)$has been computed by $\mathrm{Lu}[52$, Proposition 3.5]. In this appendix, we recall the definition of the $\zeta$ function regularization and explain the meaning of (1.1.1).

For a sequence $\left\{\lambda_{n}\right\}_{n=1}^{\infty}$ of complex numbers, the associated $\zeta$-function is defined to be $f(s)=\sum_{n=1}^{\infty} \lambda_{n}^{-s}$. If $f(s)$ can be analytically continued to a holomorphic function around $s=0$, we define the $\zeta$-regularized product of $\left\{\lambda_{n}\right\}_{n=1}^{\infty}$ to be $\exp \left(-f^{\prime}(0)\right)$ and write $\prod_{n=1}^{\infty} \lambda_{n} \sim$ $\exp \left(-f^{\prime}(0)\right)$. This is called the $\zeta$-function regularization. 
Let $\delta_{1}, \ldots, \delta_{\operatorname{dim} X}$ denotes the Chern roots of $T X$. The equation (1.1.1) follows by regarding $\delta_{i}, z$ as positive real numbers and applying the $\zeta$-function regularization to the infinite product

$$
\frac{1}{e_{S^{1}}\left(\mathcal{N}_{+}\right)}=\prod_{i=1}^{\operatorname{dim} X} \prod_{n=1}^{\infty} \frac{1}{\delta_{i}+n z} .
$$

Indeed, the $\zeta$-function regularization gives

$$
\prod_{n=1}^{\infty} \frac{1}{\delta_{i}+n z} \sim \sqrt{\frac{z}{2 \pi}} z^{\delta_{i} / z} \Gamma\left(1+\delta_{i} / z\right)
$$

where we note that the associated $\zeta$-function is the Hurwitz zeta function $z^{s} \zeta(-s ; \delta / z+1)$.

Acknowledgments: We thank Boris Dubrovin, Ionut Ciocan-Fontanine, Kohei Iwaki, Bumsig Kim, Etienne Mann, Anton Mellit, Kaoru Ono for many useful suggestions and simplifications. We also thank anonymous referees for valuable comments and suggestions.

Funding Sources: S.G. was supported by grant MK-1297.2014.1; AG Laboratory NRUHSE, RF government grant, ag. 11.G34.31.0023; Grant of Leading Scientific Schools (N.Sh. 2998.2014.1); World Premier International Research Center Initiative (WPI Initiative), MEXT, Japan; and JSPS KAKENHI Grant Number 10554503. H.I. was supported by JSPS KAKENHI (Kinban-C) Grant Number 25400069; JSPS KAKENHI (Wakate-B) Grant Number 19740039; and EPSRC (EP/E022162/1).

\section{REFERENCES}

1. Mark J. Ablowitz and Athanassios S. Fokas, Complex variables: introduction and applications. Second edition. Cambridge Texts in Applied Mathematics. Cambridge University Press, Cambridge, 2003.

2. Gert Almkvist, Duco van Straten and Wadim Zudilin: Apéry limits of differential equations of order 4 and 5, Yui, Noriko (ed.) et al., Modular forms and string duality. Proceedings of a workshop, Banff, Canada, June 3-8, 2006. Providence, RI: American Mathematical Society (AMS); Toronto: The Fields Institute for Research in Mathematical Sciences. Fields Institute Communications 54, 105-123 (2008)., 2008.

3. M. F. Atiyah: Circular symmetry and stationary phase approximation, pp. 43-59 in Colloque en l'honneur de Laurent Schwartz (École Polytechnique, Palaiseau, 30 May - 3 June 1983). Astérisque 131. Société mathématique de France (Paris), 1985.

4. Welner Balser, Wolfgang B. Jurkat, and Donald A. Lutz, Birkhoff invariants and Stokes' multipliers for meromorphic linear differential equations, J. Math. Anal. Appl. 71 (1979), no. 1, 48-94.

5. Welner Balser, Wolfgang B. Jurkat, and Donald A. Lutz, On the reduction of connection problems for differential equations with an irregular singular point to ones with only regular singularities. I. SIAM J. Math. Anal. 12 (1981), no. 5, 691-721.

6. Alexander Beilinson: Coherent sheaves on $\mathbb{P}^{n}$ and problems in linear algebra, Funktsional. Anal. $i$ Prilozhen., 12(3):68-69, 1978.

7. Alexey Bondal and Alexander Polishchuk: Homological properties of associative algebras: the method of helices, Russian Academy of Sciences. Izvestiya Mathematics, 1994, 42:2, 219-260.

8. Aaron Bertram: Quantum Schubert calculus, Adv. Math. 128 (1997), no. 2, 289-305, arXiv:alggeom $/ 9410024$

9. Aaron Bertram, Ionut Ciocan-Fontanine, and Bumsig Kim: Two proofs of a conjecture of Hori and Vafa, Duke Math. J. 126, No. 1, 101-136 (2005), arXiv:math.AG/0304403.

10. Anders Skovsted Buch: Quantum cohomology of Grassmannians, Compositio Math. 137 (2003), no. 2, 227-235, arXiv:math/0106268.

11. Lev Borisov and Richard Paul Horja: Mellin-Barnes integrals as Fourier-Mukai transforms, Adv. Math. 207 (2006), no. 2, 876-927, arXiv:math/0510486.

12. Tom Bridgeland and Valerio Toledano-Laredo, Stokes factors and multilogarithms, J. Reine Angew. Math. 682 (2013), 89-128, arXiv:1006.4623v6.

13. Philip Candelas, Xenia C. de la Ossa, Paul S. Green and Linda Parkes: An exactly soluble superconformal theory from a mirror pair of Calabi-Yau manifolds, Phys. Lett. B 258 (1991), no.1-2, pp.118-126. 
14. Ionut Ciocan-Fontanine, Bumsig Kim, and Claude Sabbah: The abelian/non-abelian correspondence and Frobenius manifolds, Invent. Math. 171 (2008), no. 2, 301-343, arXiv:math/0610265.

15. Tom Coates, Alessio Corti, Sergey Galkin, Vasily Golyshev, Al Kasprzyk, Mirror symmetry and Fano manifolds, European Congress of Mathematics (Kraków, 2-7 July, 2012), November 2013 (824 pages), pp. 285-300, ISBN 978-3-03719-120-0, DOI 10.4171/120-1/16, arXiv:1212.1722.

16. Boris Dubrovin: Geometry of $2 D$ topological field theories, Francaviglia, M. (ed.) et al., Integrable systems and quantum groups. Lectures given at the 1st session of the Centro Internazionale Matematico Estivo (CIME) held in Montecatini Terme, Italy, June 14-22, 1993. Berlin: Springer-Verlag. Lect. Notes Math. 1620, 120-348 (1996)., 1996, arXiv:hep-th/9407018.

17. Boris Dubrovin: Painlevé transcendents and two-dimensional topological field theory, In The Painlevé property, CRM Ser. Math. Phys., 287-412. Springer, New York, 1999, arXiv:math/9803107.

18. Boris Dubrovin: Geometry and analytic theory of Frobenius manifolds, In Proceedings of the International Congress of Mathematicians, Vol. II (Berlin, 1998), 315-326, arXiv:math/9807034.

19. Boris Dubrovin Quantum cohomology and isomonodromic deformation, Lecture at "Recent Progress in the Theory of Painlevé Equations: Algebraic, asymptotic and topological aspects", Strasbourg, November 2013.

20. Christian van Enckevort and Duco van Straten: Monodromy calculations of fourth order equations of Calabi-Yau type, Mirror symmetry. V, 539-559, AMS/IP Stud. Adv. Math., 38, Amer. Math. Soc., Providence, RI, 2006, arXiv:math/0412539.

21. William Fulton: Young tableaux, With applications to representation theory and geometry. London Mathematical Society Student Texts, 35. Cambridge University Press, Cambridge, 1997.

22. William Fulton and Chris Woodward: On the quantum product of Schubert classes, J. Algebr. Geom. 13 (2004), no. 4, 641-661, arXiv:math/0112183.

23. Sergey Galkin: Apery constants of homogeneous varieties, preprint SFB45 (2008).

24. Sergey Galkin: The conifold point, arXiv:1404.7388.

25. Sergey Galkin and Hiroshi Iritani, Gamma conjecture via mirror symmetry, arXiv:1508.00719.

26. Victor Ginzburg: Perverse sheaves on a Loop group and Langlands' duality, arXiv:alg-geom/9511007.

27. Alexander B. Givental: Homological geometry and mirror symmetry, In: Proceedings of the ICM, Zürich, 1994, Birkhäuser, Basel, 1995, vol 1, pp.472-480.

28. Alexander B. Givental: Elliptic Gromov-Witten Invariants and the generalized mirror conjecture, In: Integrable Systems and algebraic geometry (Kobe/Kyoto 1997), pp.107-155, World Sci. Publ., River Edge, NJ, 1998.

29. Alexander B. Givental: Symplectic geometry of Frobenius structures, Frobenius manifolds, 91-112, Aspects Math., E36, Friedr. Vieweg, Wiesbaden, 2004, arXiv:math/0305409.

30. Vasily Golyshev: Riemann-Roch variations, Izvestiya:Mathematics, 2001, 65:5, 853-881, link.

31. Vasily Golyshev: Deresonating a Tate period, arXiv:0908.1458.

32. Vasily Golyshev and Laurent Manivel: Quantum cohomology and the Satake isomorphism, arXiv:1106.3120.

33. Vasily Golyshev and Anton Mellit: Gamma structures and Gauss's contiguity, J. Geom. Phys. 78 (2014), 12-18, arXiv:0902.2003

34. Alexey Gorodentsev and Sergey Kuleshov: Helix theory, Mosc. Math. J., 2004, 4:2, 377-440.

35. Davide Guzzetti: Stokes matrices and monodromy of the quantum cohomology of projective spaces, Comm. Math. Phys. 207 (1999), no. 2, 341-383, arXiv:math/9904099.

36. James Halversona, Hans Jockers, Joshua M. Lapan and David R. Morrison: Perturbative Corrections to Kähler Moduli Spaces, arXiv:1308.2157.

37. Claus Hertling and Yuri I. Manin: Unfoldings of meromorphic connections and a construction of Frobenius manifolds, Frobenius manifolds, 113-144, Aspects Math., E36, Vieweg, Wiesbaden, 2004, arXiv:math/0207089

38. Claus Hertling and Christian Sevenheck, Nilpotent orbits of a generalization of Hodge structures, J. Reine Angew. Math. 609 (2007), pp.23-80.

39. Kentaro Hori and Mauricio Romo: Exact results in two-dimensional $(2,2)$ sypersymmetric gauge theory with boundary, arXiv:1308.2438.

40. Richard Paul Horja: Hypergeometric functions and mirror symmetry in toric varieties, arXiv:math/9912109. 
41. Shinobu Hosono: Central charges, symplectic forms, and hypergeometric series in local mirror symmetry, Mirror symmetry. V, pp.405-439, AMS/IP Stud. Adv. Math., 38, Amer. Math. Soc., Providence, RI, 2006, arXiv:hep-th/0404043.

42. Shinobu Hosono, Albrecht Klemm, Stefan Theisen and Shing-Tung Yau: Mirror symmetry, mirror map and applications to complete intersection Calabi-Yau spaces, Nuclear Phys. B 433 (1995), no. 3, 501-552, arXiv:hep-th/9406055.

43. Masuo Hukuhara, Sur les points singuliers des équations différentielles linéaires II, III, Jour. Fac. Sci. Hokkaido Imp. Univ., 5 (1937), pp.123-166; Mem. Fac. Sci. Kyushu Imp. Univ., 2 (1941), pp.125-137.

44. Hiroshi Iritani: Real and integral structures in quantum cohomology I: toric orbifolds, arXiv:0712.2204.

45. Hiroshi Iritani: An integral structure in quantum cohomology and mirror symmetry for toric orbifolds, Adv. Math. 222 (2009), no. 3, 1016-1079, arXiv:0903.1463.

46. Hiroshi Iritani: $t t^{*}$-geometry in quantum cohomology, arXiv:0906.1307.

47. Mikhail Kapranov: The derived category of coherent sheaves on Grassmann varieties, Funktsional. Anal. i Prilozhen. 17 (1983), no. 2, 78-79.

48. Mikhail Kapranov: On the derived categories of coherent sheaves on some homogeneous spaces, Invent. Math. 92, 479-508 (1988).

49. Ludmil Katzarkov, Maxim Kontsevich, and Tony Pantev, Hodge theoretic aspects of mirror symmetry, arXiv:0806.0107.

50. Bumsig Kim and Claude Sabbah: Quantum cohomology of the Grassmannian and alternate ThomSebastiani, Compos. Math. 144 (2008), no.1, 221-246, arXiv:math/0611475.

51. Anatoly S. Libgober, Chern classes and the periods of mirrors, Math. Res. Lett., 6 (1999), 141-149, arXiv:math/9803119.

52. Rongmin Lu, The $\widehat{\Gamma}$-genus and a regularization of an $S^{1}$-equivariant Euler class, J. Phys. A 41 (2008), no.42, 425204 (13pp), arXiv:0804.2714.

53. J. Malmquist, Sur l'étude analytique des solutions d'un système d'équations différentielles dans le voisinage d'un point singulier d'indétermination I, II, III, Acta Math. 73, (1940), pp.87-129; Acta Math. 74, (1941), pp.1-64; Acta Math. 74, (1941), pp.109-128.

54. Yuri I. Manin, Frobenius manifolds, quantum cohomology, and moduli spaces, American Mathematical Society Colloquium Publications, 47. American Mathematical Society, Providence, RI, 1999.

55. Cornelis S. Meijer, On the G-function. I-VIII, Indagationes Math. 8, pp.124-134, pp.213-225, pp.312-324, pp.391-400, pp.468-475, pp.595-602, pp.661-670, pp.713-723, (1946).

56. Rahul Pandharipande, Rational curves on hypersurfaces (after A. Givental), Séminaire Bourbaki. Vol. 1997/98, Astérisque, 252, 1998, Exp. No. 848, 5, 307-340, arXiv:math/9806133.

57. Claude Sabbah, Isomonodromic deformations and Frobenius manifolds. An Introduction, Translated from the 2002 French edition, Universitext, Springer-Verlag London, Ltd., London; EDP Sciences, Les Ulis, 2007.

58. Yasutaka Sibuya, Simplification of a system of linear ordinary differential equations about a singular point, Funkcialaj Ekvacioj, 4 (1962), pp.29-56.

59. Yasutaka Sibuya, Linear differential equations in the complex domain: problems of analytic continuation, Translated from the Japanese by the author. Translations of Mathematical Monographs, 82. American Mathematical Society, Providence, RI, 1990.

60. Susumu Tanabé, Invariant of the hypergeometric group associated to the quantum cohomology of the projective space, Bulletin des Sciences Mathématiques, Vol 128, 10 (2004), pp.811-827, arXiv:math/0201090.

61. Constantin Teleman, The structure of $2 D$ semisimple field theories, Invent. Math. 188, No. 3, 525-588 (2012), arXiv:0712.0160.

62. Waldemar J. Trjitzinsky, Analytic theory of linear differential equations, Acta Math. 62 (1933), no.1, pp.167-226.

63. Hugh L. Turrittin, Convergent solutions of ordinary linear homogeneous differential equations in the neighborhood of an irregular singular point, Acta Math. 93, (1955). pp.27-66.

64. Kazushi Ueda: Stokes matrices for the quantum cohomologies of Grassmannians, Int. Math. Res. Not. 2005, No. 34, 2075-2086 (2005), arXiv:math/0503355.

65. Wolfgang Wasow: Asymptotic expansions for ordinary differential equations, Pure and Applied Mathematics, Vol. XIV Interscience Publishers John Wiley \& Sons, Inc., New York-London-Sydney 1965.

66. Masaaki Yoshida and Kyoichi Takano, Local theory of Fuchsian systems. I. Proc. Japan Acad. 51 (1975), no. 4, 219-223. 
67. Eric Zaslow: Solitons and helices: the search for a math-physics bridge, Comm. Math. Phys., 175(2):337375, 1996. arXiv:hep-th/9408133.

National Research University Higher School of Economics, Faculty of Mathematics and Laboratory of Algebraic Geometry, Vavilova str. 7, Moscow 117312, Russia

E-mail address: sergey.galkin@phystech.edu

Algebra and Number Theory Sector, Institute for Information Transmission Problems, BolSHOY Karetny PER.19, Moscow 127994, Russia

E-mail address: golyshev@mccme.ru

Department of Mathematics, Graduate School of Science, Kyoto University, KitashirakawaOiWAKE-CHO, SAKYO-KU, KYOTO, 606-8502, JAPAN

E-mail address: iritani@math.kyoto-u.ac.jp 